\title{
Rearrangements of Bicyclic Nitrones to Lactams: Comparison of Photochemical and Modified Barton Conditions
}

Yibin Zeng, Brenton T. Smith, John Hershberger, and Jeffrey Aubé*

Department of Medicinal Chemistry, 1251 Wescoe Hall Drive, Room 4070, Malott Hall, University of Kansas, Lawrence, Kansas 66045-2506

$$
\text { jaube@ku.edu }
$$

\section{Supporting Information}

Experimental Section

Copies of ${ }^{1} \mathrm{H}$ and ${ }^{13} \mathrm{C}$ spectrum of new compounds

\section{Corresponding author:}

Professor Jeffrey Aubé

Department of Medicinal Chemistry

1251 Wescoe Hall Drive, Room 4070

Malott Hall

University of Kansas

Lawrence, Kansas 66045-2506

Tel 785.864.4496

Fax 785.864.5326

E-mail: jaube@ku.edu 
General Information. General procedures for the preparation of iodoalkyl or bromoalkyl ketone intermediates have been described. ${ }^{1-3}$ The following lactams: 15-17, 19-20, 24, 27 and the vinylogous amide 35 are known compounds. ${ }^{2,3}$

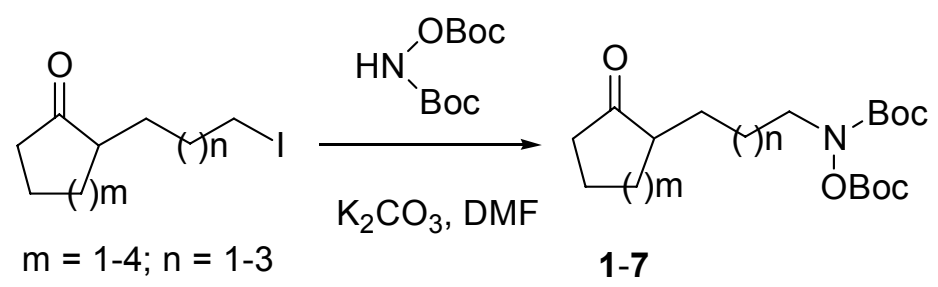

\section{General Procedure for preparation of the $\mathrm{N}, \mathrm{O}$-di(butoxycarbamyl)hydroxyl-} amino-alkyl)ketones. Iodoalkyl or bromoalkyl ketone (5-12 mmol) was dissolved in 15$20 \mathrm{~mL}$ of DMF, $N, O$-di(butoxycarbamyl)hydroxylamine ${ }^{4}$ (0.83-0.95 equiv) and $\mathrm{K}_{2} \mathrm{CO}_{3}$ (1.2 equiv) were added at room temperature and the reaction mixture was stirred for $24 \mathrm{~h}$. The reaction was partitioned between water and EtOAc. The organic layer was dried $\left(\mathrm{Na}_{2} \mathrm{SO}_{4}\right)$, filtered and concentrated to give an oil. Chromatography (10\% EtOAc/hexane) afforded $77-92 \%$ of $\mathrm{N}, \mathrm{O}$-di(butoxycarbamyl)hydroxylaminoalkyl)ketones isolated as oils.

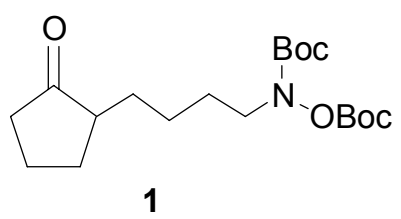

( \pm )-2-(4'-N,O-Di(butoxycarbamyl)hydroxylaminobutyl)cyclopentanone (1). $( \pm)$-2-(4'-Iodobutyl)cyclopentanone ${ }^{2}(3.2 \mathrm{~g}, 12 \mathrm{mmol})$ afforded $2.9 \mathrm{~g}(78 \%)$ of $\mathbf{1}$ isolated as an oil. TLC (10:1 hexane/EtOAc): $\mathrm{R}_{f}=0.32 .{ }^{1} \mathrm{H}$ NMR $\left(400 \mathrm{MHz}, \mathrm{CDCl}_{3}\right) \delta 1.20-1.42$ 
(m, 3H), 1.47 (s, 9H), 1.52 (s, 9H), 1.59 (m, 2H), 1.70-2.32 (complex, 8H), 3.57 (br s, 2H); ${ }^{13} \mathrm{C} \mathrm{NMR}\left(100.6 \mathrm{MHz}, \mathrm{CDCl}_{3}\right) \delta$ 20.9, 24.8, 27.2, 27.8, 28.3, 29.5, 29.7, 38.3, 49.3, 50.1, 82.4, 84.9, 152.5, 155.1, 221.5; IR (neat) 1796, $1730 \mathrm{~cm}^{-1}$; MS (ESI) $\mathrm{m} / z 372$ $\left(\mathrm{M}^{+}+1\right)$; HRMS calcd for $\mathrm{C}_{19} \mathrm{H}_{34} \mathrm{NO}_{6}\left(\mathrm{M}^{+}+\mathrm{H}\right)$ : 372.2386, found 372.2370.

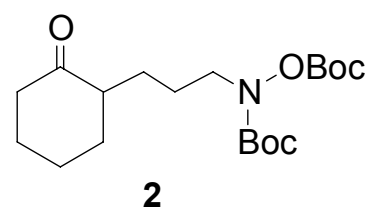

(士)-2-(4'-N,O-Di(butoxycarbamyl)hydroxylaminopropyl)cyclohexanone (2). ( \pm -2-(4'-Iodopropyl)cyclohexanone ${ }^{2}(2.0 \mathrm{~g}, 7 \mathrm{mmol})$ afforded $1.9 \mathrm{~g}(79 \%)$ of 2 isolated as an oil. TLC (10:1 hexane/EtOAc): $\mathrm{R}_{f}=0.35 .{ }^{1} \mathrm{H}$ NMR $\left(400 \mathrm{MHz}, \mathrm{CDCl}_{3}\right) \delta 1.20-1.40$ (m, 2H), 1.46 (s, 9H), 1.51 (s, 9H), 1.58-2.38 (complex, 11H), 3.55 (br s, $2 \mathrm{H}) ;{ }^{13} \mathrm{C}$ NMR $\left(100.6 \mathrm{MHz}, \mathrm{CDCl}_{3}\right) \delta 25.1,25.3,26.8,28.0,28.4,28.5,34.4,42.4,50.6,50.7,82.6$, 85.1, 152.7, 155.3, 213.4; IR (neat) 1789, $1716 \mathrm{~cm}^{-1}$; MS (ESI) $\mathrm{m} / z 372\left(\mathrm{M}^{+}+1\right)$; HRMS calcd for $\mathrm{C}_{19} \mathrm{H}_{34} \mathrm{NO}_{6}\left(\mathrm{M}^{+}+\mathrm{H}\right): 372.2386$, found 372.2380.

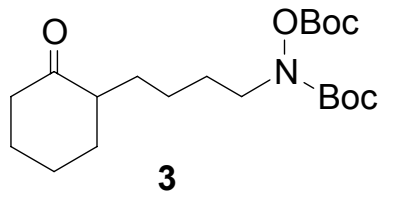

( \pm )-2-(4'-N,O-Di(butoxycarbamyl)hydroxylaminopentyl)cyclohexanone (3). ( \pm )-2-(4'-Iodobutyl)cyclohexanone ${ }^{2}(1.2 \mathrm{~g}, 4.6 \mathrm{mmol})$ afforded $1.36 \mathrm{~g}(80 \%)$ of $\mathbf{3}$ isolated as an oil. TLC (10:1 hexane/EtOAc): $\mathrm{R}_{f}=0.36 .{ }^{1} \mathrm{H}$ NMR $\left(400 \mathrm{MHz}, \mathrm{CDCl}_{3}\right) \delta 1.15-1.35$ (m, 4H), $1.44(\mathrm{~s}, 9 \mathrm{H}), 1.50(\mathrm{~s}, 9 \mathrm{H}), 1.54-2.37$ (complex, 11H), 3.54 (br s, $2 \mathrm{H}) ;{ }^{13} \mathrm{C}$ NMR $\left(100.6 \mathrm{MHz}, \mathrm{CDCl}_{3}\right) \delta$ 24.5, 25.3, 27.5, 28.0, 28.4, 28.5, 29.3, 34.2, 42.4, 50.4, 51.0, 
82.5, 85.0, 152.7, 155.3, 213.6; IR (neat) 1781, $1716 \mathrm{~cm}^{-1}$; MS (ESI) $\mathrm{m} / z 386\left(\mathrm{M}^{+}+1\right)$; HRMS calcd for $\mathrm{C}_{20} \mathrm{H}_{36} \mathrm{NO}_{6}\left(\mathrm{M}^{+}+\mathrm{H}\right): 386.2543$, found 386.2564 .

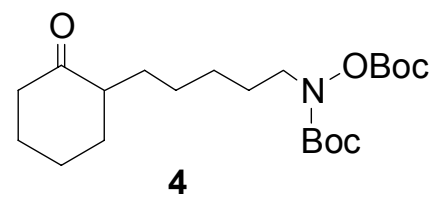

(士)-2-(4'-N,O-di(butoxycarbamyl)hydroxylaminopentyl)cyclohexanone (4). $( \pm)-2-\left(4\right.$ '-Iodopentyl)cyclohexanone ${ }^{2}(1.4 \mathrm{~g}, 4.7 \mathrm{mmol})$ afforded $1.44 \mathrm{~g}(82 \%)$ of $\mathbf{4 d}$ isolated as an oil. TLC (10:1 hexane/EtOAc): $\mathrm{R}_{f}=0.37 .{ }^{1} \mathrm{H}$ NMR $\left(400 \mathrm{MHz}, \mathrm{CDCl}_{3}\right) \delta$ 1.10-1.36 (m, 6H), $1.45(\mathrm{~s}, 9 \mathrm{H}), 1.50(\mathrm{~s}, 9 \mathrm{H}), 1.54-2.36$ (complex, 11H), 3.53 (br s, 2H); ${ }^{13} \mathrm{C}$ NMR (100.6 MHz, $\left.\mathrm{CDCl}_{3}\right) \delta 25.2,27.1,27.2,27.3,28.0,28.4,28.5,29.6,34.3,42.4$, 50.4, 51.0, 82.5, 85.0, 152.7, 155.3, 213.8; IR (neat) 1789, $1716 \mathrm{~cm}^{-1}$; MS (ESI) $\mathrm{m} / z 400$ $\left(\mathrm{M}^{+}+1\right)$; HRMS calcd for $\mathrm{C}_{21} \mathrm{H}_{38} \mathrm{NO}_{6}\left(\mathrm{M}^{+}+\mathrm{H}\right)$ : 400.2699, found 400.2696 .

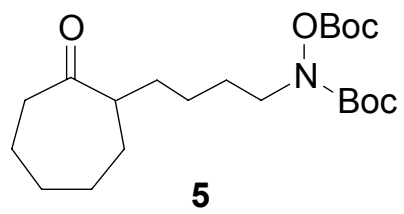

(士)-2-(4'-N,O-di(butoxycarbamyl)hydroxylaminobutyl)cycloheptanone (5).

$( \pm)$-2-(4'-Iodobutyl)cycloheptanone ${ }^{2}(1.23 \mathrm{~g}, 4.6 \mathrm{mmol})$ afforded $1.36 \mathrm{~g}(80 \%)$ of $\mathbf{5}$ isolated as an oil. TLC (10:1 hexane/EtOAc): $\mathrm{R}_{f}=0.39 .{ }^{1} \mathrm{H}$ NMR $\left(400 \mathrm{MHz}, \mathrm{CDCl}_{3}\right) \delta$ 1.25-1.38 (m, 7H), 1.48 (s, 9H), 1.54 (s, 9H), 1.57-2.53 (complex, 10H), 3.56 (br s, 2H); ${ }^{13} \mathrm{C}$ NMR $\left(100.6 \mathrm{MHz}, \mathrm{CDCl}_{3}\right) \delta 24.7,24.9,27.5,28.0,28.5,28.9,29.9,31.6,32.3,43.2$, 
50.4, 52.6, 82.6, 85.1, 152.7, 155.3, 216.7; IR (neat) $1781,1716 \mathrm{~cm}^{-1}$; MS (ESI) $\mathrm{m} / z 400$ $\left(\mathrm{M}^{+}+1\right)$; HRMS calcd for $\mathrm{C}_{21} \mathrm{H}_{38} \mathrm{NO}_{6}\left(\mathrm{M}^{+}+\mathrm{H}\right)$ : 400.2699, found 400.2680.

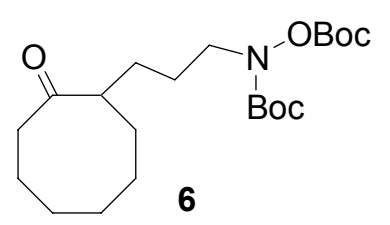

(士)-2-(4'-N,O-di(butoxycarbamyl)hydroxylaminopropyl)cyclooctanone (6).

( \pm )-2-(4'-Iodopropyl)cyclooctanone ${ }^{2}(1.2 \mathrm{~g}, 4.5 \mathrm{mmol})$ afforded $1.4 \mathrm{~g}(82 \%)$ of 6 isolated as an oil. TLC (10:1 hexane/EtOAc): $\mathrm{R}_{f}=0.38 .{ }^{1} \mathrm{H}$ NMR $\left(400 \mathrm{MHz}, \mathrm{CDCl}_{3}\right) \delta$ 1.16-1.43 (m, 6H), 1.47 (s, 9H), 1.53 (s, 9H), 1.55-2.57 (complex, 11H), 3.54 (br s, $2 \mathrm{H}) ;{ }^{13} \mathrm{C}$ NMR $\left(100.6 \mathrm{MHz}, \mathrm{CDCl}_{3}\right) \delta 25.1,25.4,25.8,26.1,27.8,28.0,28.5,29.7,33.2,42.5,50.3$, 50.5, 82.6, 85.1, 152.6, 155.3, 220.3; IR (neat) 1781, $1701 \mathrm{~cm}^{-1}$; MS (ESI) $\mathrm{m} / z 400$ $\left(\mathrm{M}^{+}+1\right)$; HRMS calcd for $\mathrm{C}_{21} \mathrm{H}_{38} \mathrm{NO}_{6}\left(\mathrm{M}^{+}+\mathrm{H}\right)$ : 400.2699, found 400.2678.

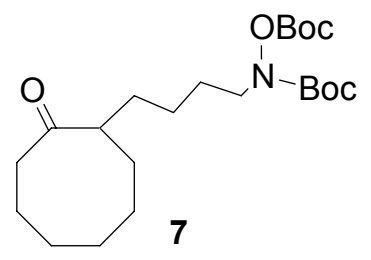

( \pm )-2-(4'-N,O-Di(butoxycarbamyl)hydroxylaminobutyl)cyclooctanone (7).

$( \pm)-2-\left(4\right.$ '-Iodobutyl)cyclooctanone ${ }^{2}(3.4 \mathrm{~g}, 11 \mathrm{mmol})$ afforded $3.3 \mathrm{~g}(85 \%)$ of 7 isolated as an oil. TLC (10:1 hexane/EtOAc): $\mathrm{R}_{f}=0.42 .{ }^{1} \mathrm{H}$ NMR $\left(400 \mathrm{MHz}, \mathrm{CDCl}_{3}\right) \delta$ 1.15-1.44 (m, 6H), 1.47 (s, 9H), 1.53 (s, 9H), 1.57-2.60 (complex, 13H), 3.54 (br s, $2 \mathrm{H}) ;{ }^{13} \mathrm{C}$ NMR 
$\left(100.6 \mathrm{MHz}, \mathrm{CDCl}_{3}\right) \delta 24.9,25.2,25.8,26.1,27.5,27.8,28.0,28.5,32.5,33.2,42.6$, 50.3, 50.9, 82.6, 85.1, 152.7, 155.3, 220.6; IR (neat) $1789,1716 \mathrm{~cm}^{-1}$; MS (ESI) $\mathrm{m} / z 414$ $\left(\mathrm{M}^{+}+1\right)$; HRMS calcd for $\mathrm{C}_{22} \mathrm{H}_{40} \mathrm{NO}_{6}\left(\mathrm{M}^{+}+\mathrm{H}\right)$ : 414.2865, found 414.2845.

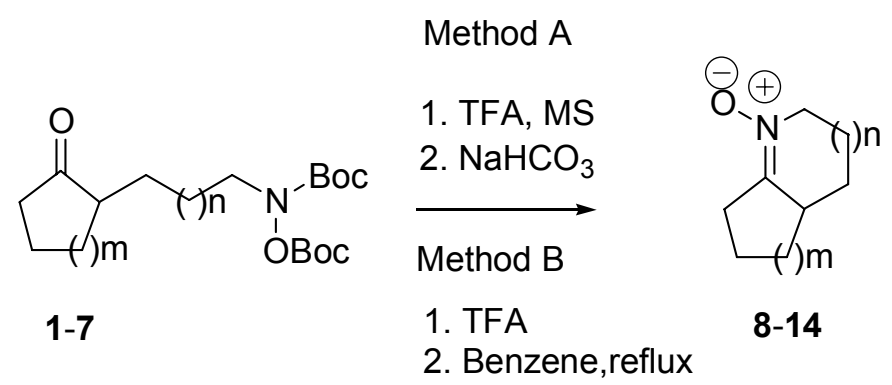

General Procedure for Preparation of Nitrones. Method A. ${ }^{1}$ N,O-DiBochydroxylaminoketone $(1-2 \mathrm{mmol})$ was dissolved in TFA $(1.5-2.5 \mathrm{~mL})$ and $\mathrm{CH}_{2} \mathrm{Cl}_{2}$ to which was added $0.5-0.8 \mathrm{~g}$ of $4 \AA$ molecular sieves. The reaction was stirred for $24 \mathrm{~h}$ and then concentrated. To the residue was added $\mathrm{CH}_{2} \mathrm{Cl}_{2}(10 \mathrm{~mL})$ and $\mathrm{NaHCO}_{3}(1.7-2.5 \mathrm{~g})$. After stirring for $2 \mathrm{~h}$, the reaction mixture was filtered through Celite and concentrated to give an oil. Flash chromatography using neutral alumina/silica gel (10\% EtOAc/hexane, followed by $1-2 \% \mathrm{MeOH} / \mathrm{CHCl}_{3}$ ) afforded nitrones in $75-90 \%$ yields.

Method B. N,O-DiBoc-hydroxyamino ketone (0.5-2 mmol) and TFA (1.0-2.5 $\mathrm{mL}$ ) were dissolved in 5-10 $\mathrm{mL}$ of $\mathrm{CH}_{2} \mathrm{Cl}_{2}$. The reaction was stirred for $1 \mathrm{~h}$, the solvent was evaporated and benzene $(15 \mathrm{~mL})$ was added. The mixture was refluxed using a Dean-Stark condenser for another $2 \mathrm{~h}$. Concentration followed by chromatography using neutral alumina/silica gel $\left(10 \%\right.$ EtOAc/Hexane, followed by $\left.1-2 \% \mathrm{MeOH} / \mathrm{CHCl}_{3}\right)$ afforded nitrones in $78-95 \%$ yields. 


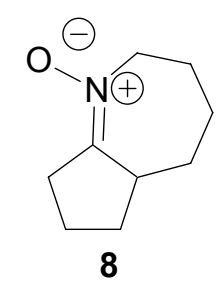

( \pm )-2,3,4,5,5a,6,7,8-Octahydrocyclopent[b]azepine-N-oxide (8). Compound 1 (400 mg, $1.1 \mathrm{mmol}$ ), using method A, gave $140 \mathrm{mg}(85 \%)$ of nitrone $\mathbf{8}$ as a white powder. $\operatorname{Mp} 28-30{ }^{\circ} \mathrm{C}$; TLC (10:1 MeOH/CHCl 3$): \mathrm{R}_{f}=0.38 .{ }^{1} \mathrm{H} \mathrm{NMR}\left(400 \mathrm{MHz}, \mathrm{CDCl}_{3}\right) \delta 1.26-$ 2.11 (complex, 10H), $2.70(\mathrm{~m}, 3 \mathrm{H}), 3.98(\mathrm{~m}, 2 \mathrm{H}) ;{ }^{13} \mathrm{C} \mathrm{NMR}\left(100.6 \mathrm{MHz}, \mathrm{CDCl}_{3}\right) \delta 23.4$, 25.7, 29.4, 31.9, 32.2, 35.3, 42.8, 66.6, 159.3; IR (neat) $1650 \mathrm{~cm}^{-1}$; MS (EI) $\mathrm{m} / z 154$ $\left(\mathrm{M}^{+}+1\right)$; HRMS calcd for $\mathrm{C}_{9} \mathrm{H}_{16} \mathrm{NO}\left(\mathrm{M}^{+}+1\right)$ : 154.1232, found 154.1251.

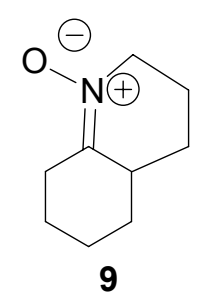

( \pm )-2,3,4,4a,5,6,7,8-Octahydroquinoline-N-oxide (9). Compound 2 (300 mg, 0.8 mmol), using method A, gave $110 \mathrm{mg}(90 \%)$ of 9 as an oil. TLC (10:1 $\left.\mathrm{MeOH} / \mathrm{CHCl}_{3}\right)$ : $\mathrm{R}_{f}=0.37 .{ }^{1} \mathrm{H} \mathrm{NMR}\left(400 \mathrm{MHz}, \mathrm{CDCl}_{3}\right) \delta$ 1.20-1.46 (complex, 4H), 1.76-1.92 (complex, 7H), $2.35(\mathrm{~m}, 1 \mathrm{H}), 3.69(\mathrm{~m}, 1 \mathrm{H}), 3.80-3.82(\mathrm{~m}, 2 \mathrm{H}) ;{ }^{13} \mathrm{C} \mathrm{NMR}\left(100.6 \mathrm{MHz}, \mathrm{CDCl}_{3}\right) \delta$ 22.1, 25.2, 25.3, 26.4, 27.1, 34.1, 38.5, 58.9, 151.7; IR (neat) $1643 \mathrm{~cm}^{-1}$; MS (CI) $\mathrm{m} / z 154$ $\left(\mathrm{M}^{+}+1\right)$; HRMS calcd for $\mathrm{C}_{9} \mathrm{H}_{16} \mathrm{NO}\left(\mathrm{M}^{+}+1\right)$ : 154.1232, found 154.1232. 


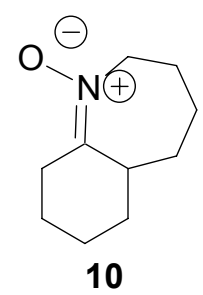

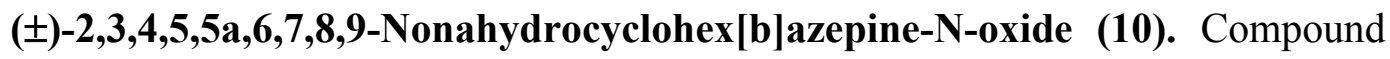
3 (526 mg, $1.36 \mathrm{mmol})$, using method A, gave $183 \mathrm{mg}(80 \%)$ of $\mathbf{1 0}$ as an oil. TLC (10:1 $\left.\mathrm{MeOH} / \mathrm{CHCl}_{3}\right): \mathrm{R}_{f}=0.35 .{ }^{1} \mathrm{H} \mathrm{NMR}\left(400 \mathrm{MHz}, \mathrm{CDCl}_{3}\right) \delta 1.46-1.60$ (complex, 6H), $1.65-$ 1.80 (complex, 5H), $1.94(\mathrm{~m}, 1 \mathrm{H}), 2.38(\mathrm{~m}, 1 \mathrm{H}), 2.79(\mathrm{~m}, 1 \mathrm{H}), 3.00-3.06(\mathrm{dt}, J=5.6$, $19.5 \mathrm{~Hz}, 1 \mathrm{H}), 3.98(\mathrm{dd}, J=5.5,15.8 \mathrm{~Hz}, 1 \mathrm{H}), 4.30-4.36(\mathrm{t}, J=11.9 \mathrm{~Hz}, 1 \mathrm{H}) ;{ }^{13} \mathrm{C} \mathrm{NMR}$ $\left(100.6 \mathrm{MHz} \mathrm{CDCl}_{3}\right) \delta 20.7,21.9,24.6,25.5,29.3,32.1,32.2,38.4,64.1,152.6$; IR (neat) $1650 \mathrm{~cm}^{-1}$; MS (CI) m/z $168\left(\mathrm{M}^{+}+1\right)$; HRMS calcd for $\mathrm{C}_{10} \mathrm{H}_{18} \mathrm{NO}\left(\mathrm{M}^{+}+1\right): 168.1388$, found 168.1381.

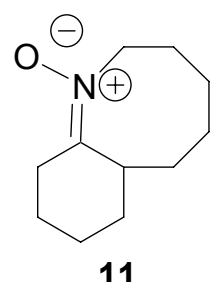

( \pm )-2,3,4,5,6,6a,7,8,9,10-Decahydrocyclohex[b]azocine-N-oxide (11). Compound 4 (500 mg, $1.25 \mathrm{mmol})$, using method A, gave $200 \mathrm{mg}(88 \%)$ of 11 as an oil. TLC (10:1 $\left.\mathrm{MeOH} / \mathrm{CHCl}_{3}\right): \mathrm{R}_{f}=0.36 .{ }^{1} \mathrm{H}$ NMR $\left(400 \mathrm{MHz}, \mathrm{CDCl}_{3}\right) \delta 1.14-2.10($ complex, $15 \mathrm{H}), 3.01$ (m, 1H), $3.53(\mathrm{~m}, 1 \mathrm{H}), 3.80(\mathrm{dt}, J=3.7,12.8 \mathrm{~Hz}, 1 \mathrm{H}), 4.37(\mathrm{ddd}, J=2.2,12.8 \mathrm{~Hz}, 1 \mathrm{H})$; ${ }^{13} \mathrm{C}$ NMR $\left(100.6 \mathrm{MHz}, \mathrm{CDCl}_{3}\right) \delta 20.1,23.9,24.3,25.7,26.4,30.0,30.9,36.1,36.7,60.4$, 
152.4; IR (neat) $1658 \mathrm{~cm}^{-1}$; MS (EI) $\mathrm{m} / z 182\left(\mathrm{M}^{+}+1\right)$; HRMS calcd for $\mathrm{C}_{11} \mathrm{H}_{20} \mathrm{NO}$ $\left(\mathrm{M}^{+}+1\right): 182.1545$, found 182.1523.

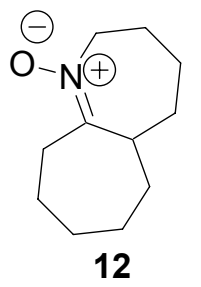

( \pm )-2,3,4,5,5a,6,7,8,9,10-Decahydrocyclohept[b]azepine-N-oxide (12). Compound 5 (400 mg, $1.0 \mathrm{mmol}$ ), using method B, gave $172 \mathrm{mg}(95 \%)$ of 12 as an oil. TLC (10:1 $\left.\mathrm{MeOH} / \mathrm{CHCl}_{3}\right): \mathrm{R}_{f}=0.38 .{ }^{1} \mathrm{H} \mathrm{NMR}\left(400 \mathrm{MHz}, \mathrm{CDCl}_{3}\right) \delta 1.14-2.10$ (complex, $\left.15 \mathrm{H}\right), 2.62$ (m, 1H), $3.41(\mathrm{~m}, 1 \mathrm{H}), 3.98(\mathrm{dt}, J=4,12.8 \mathrm{~Hz}, 1 \mathrm{H}), 4.33(\mathrm{t}, J=11.6 \mathrm{~Hz}, 1 \mathrm{H}) ;{ }^{13} \mathrm{C} \mathrm{NMR}$ $\left(100.6 \mathrm{MHz}, \mathrm{CDCl}_{3}\right) \delta$ 25.1, 26.8, 27.5, 28.4, 29.3, 30.9, 31.4, 34.9, 44.3, 64.6, 156.5; IR (neat) $1654 \mathrm{~cm}^{-1}$; MS (EI) $\mathrm{m} / z 182\left(\mathrm{M}^{+}+1\right)$; HRMS calcd for $\mathrm{C}_{11} \mathrm{H}_{20} \mathrm{NO}\left(\mathrm{M}^{+}+1\right)$ : 182.1545 , found 182.1550 .

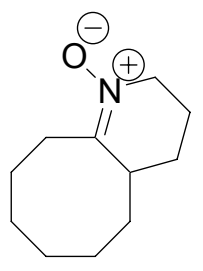

13

( \pm )-2,3,4,4a,5,6,7,8,9,10-Decahydrocycloocta[b]pyridine-N-oxide (13). Compound 6 (700 mg, $1.76 \mathrm{mmol}$ ), using method A, afforded $248 \mathrm{mg}$ (78\%) of $\mathbf{1 3}$ as an oil. TLC $\left(10: 1 \mathrm{MeOH} / \mathrm{CHCl}_{3}\right): \mathrm{R}_{f}=0.38 .{ }^{1} \mathrm{H} \mathrm{NMR}\left(400 \mathrm{MHz}, \mathrm{CDCl}_{3}\right) \delta$ 0.95-2.15 (complex, $\left.15 \mathrm{H}\right)$, 2.59(m, 1H), $3.11(\mathrm{~m}, 1 \mathrm{H}), 3.78(\mathrm{~m}, 2 \mathrm{H}) ;{ }^{13} \mathrm{C} \mathrm{NMR}\left(100.6 \mathrm{MHz}, \mathrm{CDCl}_{3}\right) \delta 22.4,24.2$, 
24.7, 25.4, 25.6, 26.2, 28.6, 30.3, 38.7, 58.8, 154.3; IR (neat) $1649 \mathrm{~cm}^{-1}$; MS (CI) $\mathrm{m} / z 182$ $\left(\mathrm{M}^{+}+1\right)$; HRMS calcd for $\mathrm{C}_{11} \mathrm{H}_{20} \mathrm{NO}\left(\mathrm{M}^{+}+1\right)$ : 182.1545 , found 182.1554 .

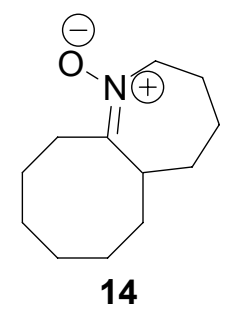

( \pm )-2,3,4,5,5a,6,7,8,9,10,11-Undecahydrocyclooct[b]azepine-N-oxide (14).

Compound 7 (410 mg, $1.0 \mathrm{mmol}$ ), using method B, gave $170 \mathrm{mg}(87 \%)$ of $\mathbf{1 4}$ as an oil. $\operatorname{TLC}\left(10: 1 \mathrm{MeOH} / \mathrm{CHCl}_{3}\right): \mathrm{R}_{f}=0.48 .{ }^{1} \mathrm{H}$ NMR $\left(400 \mathrm{MHz}, \mathrm{CDCl}_{3}\right) \delta$ 1.09-2.20 (complex, 17H), $2.48(\mathrm{~m}, 1 \mathrm{H}), 2.89(\mathrm{~m}, 1 \mathrm{H}), 3.90(\mathrm{~m}, 1 \mathrm{H}), 4.30(\mathrm{~m}, 1 \mathrm{H}) ;{ }^{13} \mathrm{C}$ NMR $(100.6 \mathrm{MHz}$, $\left.\mathrm{CDCl}_{3}\right) \delta 24.8,25.1,25.7,25.9,27.6,28.2,29.9,32.1,32.2,42.4,64.0,156.1$; IR (neat) $1687 \mathrm{~cm}^{-1}$; MS (CI) $\mathrm{m} / z 196\left(\mathrm{M}^{+}+1\right)$; HRMS calcd for $\mathrm{C}_{12} \mathrm{H}_{22} \mathrm{NO}\left(\mathrm{M}^{+}+1\right)$ : 196.1701, found 196.1709.<smiles>CCCCN(CCCCC(=O)c1ccccc1)C(=O)OCc1ccccc1</smiles>

4-N,O-Di(butoxycarbamyl)hydroxylaminobutylphenylketone (22). 4-Iodobutylphenyl-ketone $^{2}(3.4 \mathrm{~g}, 11 \mathrm{mmol})$ using the general method described above afforded $3.5 \mathrm{~g}(89 \%)$ of 22 isolated as an oil. TLC (10:1 hexane/EtOAc): $\mathrm{R}_{f}=0.33 .{ }^{1} \mathrm{H}$ NMR (400 $\left.\mathrm{MHz}, \mathrm{CDCl}_{3}\right) \delta 1.46(\mathrm{~s}, 9 \mathrm{H}), 1.51(\mathrm{~s}, 9 \mathrm{H}), 1.69(\mathrm{~m}, 2 \mathrm{H}), 1.80(\mathrm{~m}, 2 \mathrm{H}), 2.98(\mathrm{t}, J=10.8 \mathrm{~Hz}$, 2H), 3.62 (br s, 2H), 7.42-7.94 (m, 5H); ${ }^{13} \mathrm{C}$ NMR (100.6 MHz, $\left.\mathrm{CDCl}_{3}\right) \delta$ 21.7, 27.1, 27.8, $28.0,28.2,28.5,28.8,38.4,50.2,82.6,85.1,128.4,129.0,133.3,137.3,152.7,155.3$, 
200.2; IR (neat) 1782, 1710, $1690 \mathrm{~cm}^{-1}$; MS (ESI) $\mathrm{m} / z 394\left(\mathrm{M}^{+}+1\right)$; HRMS calcd for $\mathrm{C}_{21} \mathrm{H}_{32} \mathrm{NO}_{6}\left(\mathrm{M}^{+}+\mathrm{H}\right): 394.2230$, found 394.2210.

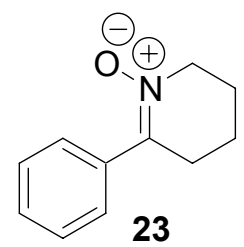

2-Phenyl-,3,4,5,6-tetrahydropyridine-N-oxide (23). Compound 22 (780 mg, 2 mmol), using method $\mathrm{B}$, gave $300 \mathrm{mg}(85 \%)$ of $\mathbf{2 3}$ as an oil. TLC $\left(10: 1 \mathrm{MeOH} / \mathrm{CHCl}_{3}\right)$ : $\mathrm{R}_{f}=0.40 .{ }^{1} \mathrm{H}$ NMR $\left(400 \mathrm{MHz}, \mathrm{CDCl}_{3}\right) \delta 1.87-1.90(\mathrm{t}, J=5.8 \mathrm{~Hz}, 2 \mathrm{H}), 2.01-2.03(\mathrm{t}, J=$ $5.0 \mathrm{~Hz}, 2 \mathrm{H}), 2.79-2.82(\mathrm{~m}, 2 \mathrm{H}), 3.99-4.02(\mathrm{~m}, 2 \mathrm{H}), 7.35-7.41(\mathrm{~m}, 3 \mathrm{H}), 8.01-8.03(\mathrm{~m}, 2 \mathrm{H})$; ${ }^{13} \mathrm{C}$ NMR $\left(100.6 \mathrm{MHz}, \mathrm{CDCl}_{3}\right) \delta 19.7,23.6,29.7,60.8,128.5,128.7,129.8,134.4$, 142.6; IR (neat) $1685 \mathrm{~cm}^{-1}$; MS (CI) $m / z 176\left(\mathrm{M}^{+}+1\right)$; HRMS calcd for $\mathrm{C}_{11} \mathrm{H}_{14} \mathrm{NO}$ $\left(\mathrm{M}^{+}+1\right):$ 176.1075, found 176.1073 .

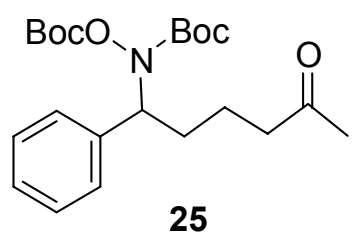

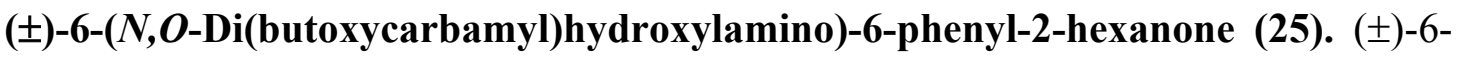
bromo-6-phenyl-2-hexanone ${ }^{3}$ (1.8 g, $7 \mathrm{mmol}$ ) using the general method described above afforded $2 \mathrm{~g}$ (77\%) of 25 isolated as an oil. TLC (10:1 hexane/EtOAc): $\mathrm{R}_{f}=0.22 .{ }^{1} \mathrm{H}$ NMR (400 MHz, $\mathrm{CDCl}_{3}$; mixture of rotamers) $\delta$ 1.22-2.03 (complex, 23H), $2.07(\mathrm{~s}, 3 \mathrm{H})$, $2.42(\mathrm{~m}, 2 \mathrm{H}), 5.14(\mathrm{~m}, 1 \mathrm{H}), 7.19-7.34(\mathrm{~m}, 5 \mathrm{H}) ;{ }^{13} \mathrm{C} \mathrm{NMR}\left(100.6 \mathrm{MHz}, \mathrm{CDCl}_{3}\right.$; mixture of rotamers) $\delta 14.5,17.2,20.8,21.1,21.2,23.0,27.7,27.9,28.4,30.1,30.4,31.4,31.9,35.0$, 
$43.5,62.2,63.6,77.3,77.6,77.9,82.6,82.9,84.5,84.7,119.6,128.2,128.4,128.7,128.9$, 137.9, 139.6, 151.8, 152.8, 154.6, 155.4, 208.6; IR (neat) 1789, $1709 \mathrm{~cm}^{-1}$; MS (ESI) $\mathrm{m} / \mathrm{z}$ $408\left(\mathrm{M}^{+}+1\right)$; HRMS calcd for $\mathrm{C}_{22} \mathrm{H}_{34} \mathrm{NO}_{6}\left(\mathrm{M}^{+}+\mathrm{H}\right)$ : 408.2386, found 408.2377.

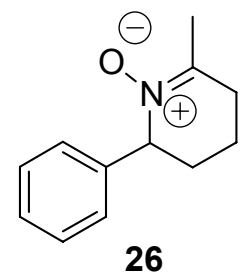

( \pm )-2-Phenyl-6-methyl-2,3,4,5-tetrahydropyridine-N-oxide (26). Compound 25 (800 mg, $2.0 \mathrm{mmol}$ ), using method A, gave $290 \mathrm{mg}$ (77\%) 26 as an oil. TLC (20:1 $\left.\mathrm{MeOH} / \mathrm{CHCl}_{3}\right): \mathrm{R}_{f}=0.09 .{ }^{1} \mathrm{H} \mathrm{NMR}\left(400 \mathrm{MHz}, \mathrm{CDCl}_{3}\right) \delta 1.59(\mathrm{~m}, 2 \mathrm{H}), 2.06(\mathrm{~m}, 1 \mathrm{H})$, 2.25 (m, 4H), 2.54 (m, 2H), 5.06 (br s, 1H), 7.13-7.33 (m, 5H); ${ }^{13} \mathrm{C}$ NMR (100.6 MHz, $\left.\mathrm{CDCl}_{3}\right) \delta 15.0,19.2,31.2,31.6,71.1,126.7,127.8,129.0,139.6,147.8$; IR (neat) 1629 $\mathrm{cm}^{-1}$; MS (CI) $m / z 190\left(\mathrm{M}^{+}+1\right)$; HRMS calcd for $\mathrm{C}_{12} \mathrm{H}_{16} \mathrm{NO}\left(\mathrm{M}^{+}+1\right)$ : 190.1232 , found 190.1223.

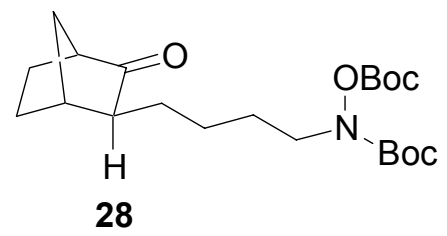

\section{3-( $\left.S^{*}\right)-\left(4^{\prime}-N, O\right.$-Di(butoxycarbamyl)hydroxylaminobutyl)-(1 $\left.R^{*}, 4 S^{*}\right)$-norcamphor}

(28). 2-( $\left.S^{*}\right)$-(4'-Iodobutyl)-( $\left(1 R^{*}, 4 S^{*}\right)$-norcamphor ${ }^{5}(1.50 \mathrm{~g}, 5.1 \mathrm{mmol})$ using the general method described above afforded $1.80 \mathrm{~g}(92 \%)$ of $\mathbf{2 8}$ isolated as an oil. TLC (10:1 hexane/EtOAc): $\mathrm{R}_{f}=0.33 .{ }^{1} \mathrm{H}$ NMR $\left(500 \mathrm{MHz}, \mathrm{CDCl}_{3}\right) \delta 1.19-1.88$ (complex, 13H), 1.44 (s, 9H), 1.50 (s, 9H), 2.39 (m, 1H), 2.49 (m, 1H), 3.54 (br s, 2H); ${ }^{13} \mathrm{C}$ NMR (100.6 
$\left.\mathrm{MHz}, \mathrm{CDCl}_{3}\right) \delta 24.4,25.7,27.3,28.0,28.3,28.5,29.0,35.2,39.4,49.9,50.2,54.3,82.5$, 85.1, 152.7, 155.3, 220.6; IR (neat) 1775, 1720, $1710 \mathrm{~cm}^{-1}$; MS (ESI) $\mathrm{m} / z 398\left(\mathrm{M}^{+}+1\right)$; HRMS calcd for $\mathrm{C}_{21} \mathrm{H}_{36} \mathrm{NO}_{6}\left(\mathrm{M}^{+}+\mathrm{H}\right): 398.2543$, found 398.2514 .

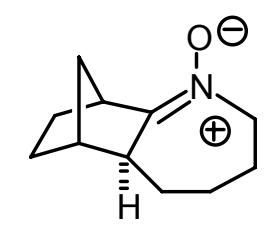

29

\section{$\left(6 \mathrm{a} S^{*}, 7 S^{*}, 10 R^{*}\right)-\left(3-\right.$ Aza-tricyclo[7.2.1.0 $\left.{ }^{127,233}\right]$ dodec-2-ene-N-oxide (29).}

Compound 28 (400 mg, $1.0 \mathrm{mmol}$ ), using method B, gave $165 \mathrm{mg}(92 \%)$ of nitrone 29 as a white powder. Mp 142-144 ${ }^{\circ} \mathrm{C}$; $\operatorname{TLC}\left(20: 1 \mathrm{MeOH} / \mathrm{CHCl}_{3}\right): R_{f}=0.05 .{ }^{1} \mathrm{H} \mathrm{NMR}(400$ $\left.\mathrm{MHz}, \mathrm{CDCl}_{3}\right) \delta 1.22(\mathrm{~m}, 3 \mathrm{H}), 1.46(\mathrm{~m}, 2 \mathrm{H}), 1.55-1.72$ (complex, 6H), $1.95(\mathrm{~m}, 1 \mathrm{H}), 2.10$ (m, 2H), $3.40($ br s, $1 \mathrm{H}), 3.80(\mathrm{~m}, 1 \mathrm{H}), 4.01(\mathrm{~m}, 1 \mathrm{H}) ;{ }^{13} \mathrm{C} \mathrm{NMR}\left(100.6 \mathrm{MHz}, \mathrm{CDCl}_{3}\right) \delta$ 25.5, 26.3, 28.5, 29.4, 30.1, 36.5, 40.9, 44.0, 50.4, 67.0, 159.4; IR (neat) $1636 \mathrm{~cm}^{-1}$; MS (CI) $m / z 180\left(\mathrm{M}^{+}+1\right)$; HRMS calcd for $\mathrm{C}_{11} \mathrm{H}_{18} \mathrm{NO}\left(\mathrm{M}^{+}+1\right): 180.1388$, found 180.1385 .

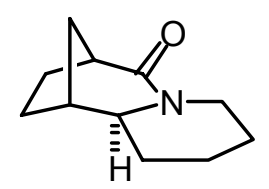

30

General Procedure for the Rearrangement of Nitrones: 10a ${ }^{*}$-Octahydro7,10-methanopyrido[1,2-a]azepin-6(2H)-one (30). Method A. ${ }^{1}$ A degassed mixture of nitrone 29 (95 $\mathrm{mg}, 0.5 \mathrm{mmol})$ in benzene $(0.025 \mathrm{M})$ was photolyzed in a capped quartz tube (254 nm, Merry-Go-Round apparatus) for $2 \mathrm{~h}$. Concentration followed by chromatography $\left(10 \%\right.$ EtOAc/hexane, followed by $\left.1-2 \% \mathrm{MeOH} / \mathrm{CHCl}_{3}\right)$ afforded $60 \mathrm{mg}$ 
$(63 \%)$ of $\mathbf{3 0}$ isolated as an oil. TLC $\left(20: 1 \mathrm{MeOH} / \mathrm{CHCl}_{3}\right): \mathrm{R}_{f}=0.23 .{ }^{1} \mathrm{H} \mathrm{NMR}(400 \mathrm{MHz}$, $\left.\mathrm{CDCl}_{3}\right) \delta$ 1.21-1.53 (complex, 7H), 1.72-1.94 (complex, 5H), $2.10(\mathrm{~m}, 1 \mathrm{H}), 2.27(\mathrm{~m}, 1 \mathrm{H})$, $2.63(\mathrm{~m}, 1 \mathrm{H}), 2.88(\mathrm{br} \mathrm{d}, J=10.6 \mathrm{~Hz}, 1 \mathrm{H}), 4.53(\mathrm{dt}, J=2.1,13.0 \mathrm{~Hz}, 1 \mathrm{H}) ;{ }^{13} \mathrm{C} \mathrm{NMR}$ $\left(100.6 \mathrm{MHz}, \mathrm{CDCl}_{3}\right) \delta 25.5,25.9,30.1,30.4,30.6,32.6,39.6,42.8,44.2,65.4,173.6$; IR (neat) $2930,1643 \mathrm{~cm}^{-1}$; MS (EI) $\mathrm{m} / z 179\left(\mathrm{M}^{+}\right)$; HRMS calcd for $\mathrm{C}_{11} \mathrm{H}_{17} \mathrm{NO}\left(\mathrm{M}^{+}+1\right)$ : 180.1376, found 180.1388 .

Method B. Nitrone 29 (280 mg, $1.6 \mathrm{mmol}$ ) was added to a solution of triflic anhydride (1.8 equiv) in $10 \mathrm{~mL}$ of $\mathrm{CH}_{2} \mathrm{Cl}_{2}$ and stirred for $2 \mathrm{~h}$. The solvent was evaporated and $25 \mathrm{~mL}$ of $15 \% \mathrm{KOH}$ was added and the reaction was allowed to stir for another $24 \mathrm{~h}$. The reaction mixture was extracted with $\mathrm{CH}_{2} \mathrm{Cl}_{2}$. The combined organic layers were dried over $\mathrm{Na}_{2} \mathrm{SO}_{4}$, filtered, and concentrated to give an oil. Chromatography ( $10 \%$ EtOAc/hexane, followed by 1-2\% $\mathrm{MeOH} / \mathrm{CHCl}_{3}$ ) afforded $265 \mathrm{mg}(95 \%)$ of $\mathbf{3 0}$ isolated as an oil.

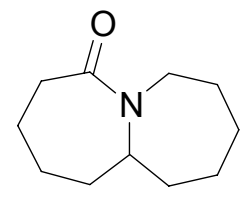

18

( \pm )-Decahydro-azapino[1,2-a]azepin-5-one (18). Method A. Nitrone 11 (100 $\mathrm{mg}, 0.56 \mathrm{mmol})$ in benzene $(20 \mathrm{~mL})$, using method A, afforded $35 \mathrm{mg}(35 \%)$ of lactam 18 as an oil. TLC $\left(10: 1 \mathrm{MeOH} / \mathrm{CHCl}_{3}\right): \mathrm{R}_{f}=0.72 .{ }^{1} \mathrm{H}$ NMR $\left(400 \mathrm{MHz}, \mathrm{CDCl}_{3}\right) \delta 1.27-$ 1.91 (complex, 15H), 2.48-2.53 (m, 1H), 2.68-2.78 (m, 2H), 3.64-3.69 (m, 1H), 4.154.20(m, 1H); ${ }^{13} \mathrm{C}$ NMR $\left(100.6 \mathrm{MHz}, \mathrm{CDCl}_{3}\right) \delta$ 23.6, 27.3, 28.8, 29.6, 30.4, 34.1, 36.0, 
37.0, 41.5, 60.2, 176.1; IR (neat) 2930, $1636 \mathrm{~cm}^{-1}$; MS (CI) $\mathrm{m} / z 182\left(\mathrm{M}^{+}+1\right)$; HRMS calcd for $\mathrm{C}_{11} \mathrm{H}_{20} \mathrm{NO}\left(\mathrm{M}^{+}+1\right)$ : 182.1545 , found 182.1547.

Method B. Nitrone 11 (80 mg, $0.44 \mathrm{mmol})$, using method B, afforded $32 \mathrm{mg}$ (40\%) lactam 18 as an oil.

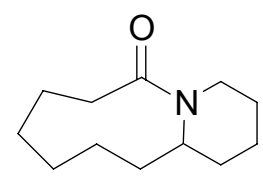

21

( \pm )-Undecahydro-6H-pyridin[1,2-a]azonin-6-one (21). Method A. Nitrone 14 (100 mg, $0.51 \mathrm{mmol})$ in benzene $(20 \mathrm{~mL})$, using method A, afforded $45 \mathrm{mg}(45 \%)$ of lactam 21 as an oil. TLC $\left(10: 1 \mathrm{MeOH} / \mathrm{CHCl}_{3}\right): \mathrm{R}_{f}=0.76 .{ }^{1} \mathrm{H}$ NMR $\left(400 \mathrm{MHz}, \mathrm{CDCl}_{3}\right) \delta$ 1.01-1.97 (complex, 15H), 1.98-2.10 (m, 1H), 2.15-2.30 (m, 1H), 2.40-2.52 (m, 3H), 4.0$4.23(\mathrm{~m}, 1 \mathrm{H}), 4.40-4.43(\mathrm{~m}, 1 \mathrm{H}) ;{ }^{13} \mathrm{C} \mathrm{NMR}\left(100.6 \mathrm{MHz}, \mathrm{CDCl}_{3}\right) \delta 19.1,20.1,25.1,25.5$, 27.9, 29.5, 29.7, 29.9, 36.6, 37.0, 52.5, 175.5; IR (neat) 2930, $1629 \mathrm{~cm}^{-1}$; MS (CI) $\mathrm{m} / \mathrm{z}$ $196\left(\mathrm{M}^{+}+1\right)$; HRMS calcd for $\mathrm{C}_{12} \mathrm{H}_{22} \mathrm{NO}\left(\mathrm{M}^{+}+1\right)$ : 196.1701, found 196.1707 .

Method B. Nitrone 14 (100 mg, $0.51 \mathrm{mmol})$, using method B, afforded $35 \mathrm{mg}$ (35\%) lactam 21 as an oil.

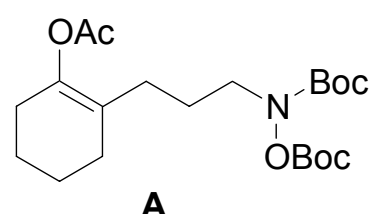

\section{1-Acetoxy-2-(3'-N,O-di(butoxycarbamyl)hydroxylaminopropyl)-1-cyclohexene}

(A). 1-Acetoxy-2-(3'-iodopropyl)-1-cyclohexene (1.4 g, $4.50 \mathrm{mmol}$, prepared from the corresponding chloride as previously described ${ }^{2}$ ) afforded $1.8 \mathrm{~g}$ (97\%) of compound $\mathbf{A}$ as 
an oil. TLC (5:1 hexane/EtOAc): $\mathrm{R}_{f}=0.39 .{ }^{1} \mathrm{H}$ NMR $\left(400 \mathrm{MHz}, \mathrm{CDCl}_{3}\right) \delta 1.49(\mathrm{~s}, 9 \mathrm{H})$, $1.51(\mathrm{~s}, 9 \mathrm{H}), 1.61-1.70$ (complex, 6H), $1.98(\mathrm{~m}, 2 \mathrm{H}), 2.05$ (br s, 2H), $2.13(\mathrm{br} \mathrm{s}, 2 \mathrm{H}), 2.14$ (s, 3H), 3.54 (br s, 2H); ${ }^{13} \mathrm{C}$ NMR $\left(100.6 \mathrm{MHz}, \mathrm{CDCl}_{3}\right) \delta 21.3,22.8,23.4,25.1,27.5$, 27.6, 28.0, 28.4, 28.5, 50.4, 82.6, 85.1, 123.6, 143.0, 152.7, 155.3, 169.8; IR (neat) 1785, 1752, $1716 \mathrm{~cm}^{-1}$; MS (ESI) $\mathrm{m} / z 414\left(\mathrm{M}^{+}+1\right)$; HRMS calcd for $\mathrm{C}_{21} \mathrm{H}_{39} \mathrm{~N}_{2} \mathrm{O}_{7}\left(\mathrm{M}^{+}+\mathrm{NH}_{4}\right)$ : 431.2757, found 431.2748 .

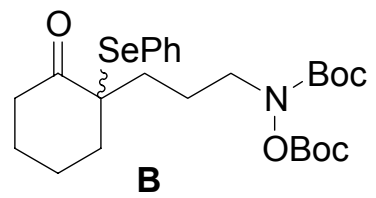

\section{2-(3'-N,O-di(butoxycarbamyl)hydroxylaminopropyl)-2-(phenylselenyl)-}

cyclohexanone (B). To silver trifluoroacetate $(0.81 \mathrm{~g}, 3.60 \mathrm{mmol})$ in $15 \mathrm{~mL}$ benzene was added the di-Boc-hydroxylamine prepared above $(1.37 \mathrm{~g}, 3.30 \mathrm{mmol})$ followed by the addition of phenylselenyl chloride in $2 \mathrm{~mL}$ of benzene $(0.70 \mathrm{~g}, 3.60 \mathrm{mmol})$. The solution was stirred for 30 min and then quenched with $\mathrm{NaHCO}_{3}\left(4.0 \mathrm{mmol}\right.$ in $1 \mathrm{~mL}$ of $\left.\mathrm{H}_{2} \mathrm{O}\right)$. The reaction mixture was filtered, dried $\left(\mathrm{Na}_{2} \mathrm{SO}_{4}\right)$, and concentrated to give an oil. Flash chromatography using silica gel (2-5\% EtOAc/hexane) afforded compound B $1.4 \mathrm{~g}$ $(80 \%)$ as an oil. TLC (5:1 hexane/EtOAc): $\mathrm{R}_{f}=0.33 .{ }^{1} \mathrm{H}$ NMR $\left(400 \mathrm{MHz}, \mathrm{CDCl}_{3}\right) \delta 1.26$ (br s, 1H), 1.47 (s, 9H), 1.54 (s, 9H), 1.59-2.10 (complex, 9H), 2.23 (br d, $J=14.1 \mathrm{~Hz}$ 1H), 2.33 (br d, $J=15.1 \mathrm{~Hz}, 1 \mathrm{H}), 3.38-3.47(\mathrm{~m}, 3 \mathrm{H}), 7.27-7.43(\mathrm{~m}, 5 \mathrm{H}) ;{ }^{13} \mathrm{C}$ NMR $(100.6$ $\left.\mathrm{MHz}, \mathrm{CDCl}_{3}\right) \delta 22.5,22.6,26.8,28.0,28.5,32.6,36.9,37.7,50.8,60.3,82.6,85.1,126.7$, 129.4, 129.8, 137.8, 152.7, 155.1, 206.7; IR (neat) $1784,1715,1693 \mathrm{~cm}^{-1}$; MS (ESI) $\mathrm{m} / \mathrm{z}$ $528\left(\mathrm{M}^{+}+1\right)$; HRMS calcd for $\mathrm{C}_{25} \mathrm{H}_{41} \mathrm{~N}_{2} \mathrm{O}_{6} \mathrm{Se}\left(\mathrm{M}^{+}+\mathrm{NH}_{4}\right)$ : 545.2130, found 545.2148. 


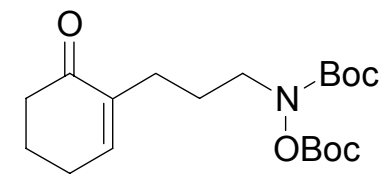

31

\section{2-(3'-N,O-di(butoxycarbamyl)hydroxylaminopropyl)cyclohex-2-enone (31).}

To a solution of selenide as prepared above $(1.0 \mathrm{~g}, 1.90 \mathrm{mmol})$ in $\mathrm{CH}_{2} \mathrm{Cl}_{2}(25 \mathrm{~mL})$ was added $\mathrm{Et}_{3} \mathrm{~N}(1 \mathrm{~mL}, 6.93 \mathrm{mmol})$ and $35 \% \mathrm{H}_{2} \mathrm{O}_{2}(8 \mathrm{~mL})$. The resulting solution was refluxed for $1 \mathrm{~h}$. Workup $\left(\mathrm{CHCl}_{3} / \mathrm{H}_{2} \mathrm{O}\right)$ followed by chromatography $(5-10 \%$ EtOAc/hexane) afforded $0.64 \mathrm{~g}(91 \%)$ of 31 as an oil. TLC (5:1 hexane/EtOAc): $\mathrm{R}_{f}=$ 0.30. ${ }^{1} \mathrm{H}$ NMR (400 MHz, $\left.\mathrm{CDCl}_{3}\right) \delta 1.47(\mathrm{~s}, 9 \mathrm{H}), 1.54(\mathrm{~s}, 9 \mathrm{H}), 1.70(\mathrm{~m}, 2 \mathrm{H}), 1.98(\mathrm{~m}$, 2H), $2.24(\mathrm{~m}, 2 \mathrm{H}), 2.34-2.43$ (complex, 4H), $3.56(\mathrm{br} \mathrm{s}, 2 \mathrm{H}), 6.77(\mathrm{t}, J=3.8 \mathrm{~Hz}, 1 \mathrm{H}) ;{ }^{13} \mathrm{C}$ NMR $\left(100.6 \mathrm{MHz}, \mathrm{CDCl}_{3}\right) \delta 23.5,26.2,26.4,27.1,28.0,28.5,38.9,50.0,82.6,85.1$, 139.1, 146.3, 152.7, 155.3, 199.8; IR (neat) 1774, 1709, $1665 \mathrm{~cm}^{-1}$; MS (ESI) $\mathrm{m} / z 370$ $\left(\mathrm{M}^{+}+1\right)$; HRMS calcd for $\mathrm{C}_{19} \mathrm{H}_{35} \mathrm{~N}_{2} \mathrm{O}_{6}\left(\mathrm{M}^{+}+\mathrm{NH}_{4}\right)$ : 387.2495 , found 387.2503.

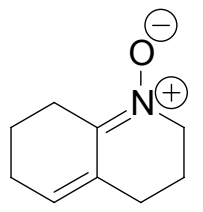

32

2,3,4,6,7,8-Hexahydroquinoline-N-oxide (32). Compound 31 (120 mg, 0.33 mmol), using method $\mathrm{B}$, gave $46 \mathrm{mg}(91 \%)$ of 32 as an oil. TLC (10:1 $\left.\mathrm{MeOH} / \mathrm{CHCl}_{3}\right)$ : $\mathrm{R}_{f}=0.39 .{ }^{1} \mathrm{H} \mathrm{NMR}\left(400 \mathrm{MHz}, \mathrm{CDCl}_{3}\right) \delta 1.78(\mathrm{p}, J=6.6 \mathrm{~Hz}, 2 \mathrm{H}), 1.96(\mathrm{p}, J=4.2 \mathrm{~Hz}$, 2H), $2.16(\mathrm{~m}, 2 \mathrm{H}), 2.37(\mathrm{~m}, 2 \mathrm{H}), 2.77(\mathrm{t}, J=6.7 \mathrm{~Hz}, 2 \mathrm{H}), 3.89(\mathrm{t}, J=5.9 \mathrm{~Hz}, 2 \mathrm{H}), 5.82(\mathrm{t}$, $J=4.5 \mathrm{~Hz}, 1 \mathrm{H}) ;{ }^{13} \mathrm{C} \mathrm{NMR}\left(100.6 \mathrm{MHz}, \mathrm{CDCl}_{3}\right) \delta 21.4,22.5,24.6,25.3,27.2,59.2$, 
128.1, 130.5, 145.7; IR (neat) $1650 \mathrm{~cm}^{-1}$; MS (CI) $\mathrm{m} / z 152\left(\mathrm{M}^{+}+1\right)$; HRMS calcd for $\mathrm{C}_{9} \mathrm{H}_{14} \mathrm{NO}\left(\mathrm{M}^{+}+1\right): 152.1075$, found 152.1070 .

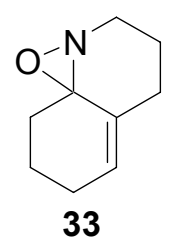

2,3,4,6,7,8-Hexahydro-oxazirino[3,2-j]quinoline (33). Nitrone 32 (50 mg, 0.33 mmol) in benzene ( $15 \mathrm{~mL})$, using method A, afforded $23 \mathrm{mg}$ (45\%) of oxaziridine 33 as an oil. TLC (10:1 MeOH/CHCl $): \mathrm{R}_{f}=0.85 .{ }^{1} \mathrm{H}$ NMR $\left(400 \mathrm{MHz}, \mathrm{CDCl}_{3}\right) \delta 1.37(\mathrm{~m}, 1 \mathrm{H})$, $1.50(\mathrm{~m}, 1 \mathrm{H}), 1.83-2.40($ complex, $8 \mathrm{H}), 3.23(\mathrm{~m}, 1 \mathrm{H}), 3.50(\mathrm{~m}, 1 \mathrm{H}), 5.99(\mathrm{t}, J=1.7 \mathrm{~Hz}$, $1 \mathrm{H}) ;{ }^{13} \mathrm{C} \mathrm{NMR}\left(100.6 \mathrm{MHz}, \mathrm{CDCl}_{3}\right) \delta 19.1,21.0,25.9,26.2,33.4,51.6,78.9,130.2$, 133.8; IR (neat) 2937, 2862, $1437 \mathrm{~cm}^{-1}$; MS (CI) $m / z 152\left(\mathrm{M}^{+}+1\right)$; HRMS calcd for $\mathrm{C}_{9} \mathrm{H}_{14} \mathrm{NO}\left(\mathrm{M}^{+}+1\right):$ 152.1075, found 152.1073 .

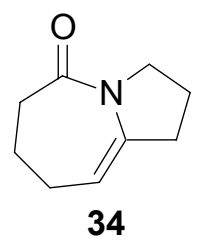

1,2,3,6,7,8-Hexahydro-pyrrolo[1,2-a]azepin-5-one (33). Method A. Nitrone 32 (50 mg, $0.33 \mathrm{mmol})$ in benzene $(15 \mathrm{~mL})$, using method A, afforded $10 \mathrm{mg}(20 \%)$ of lactam 34 as an oil. TLC (10:1 MeOH/CHCl $): \mathrm{R}_{f}=0.65 .{ }^{1} \mathrm{H} \mathrm{NMR}\left(400 \mathrm{MHz}, \mathrm{CDCl}_{3}\right) \delta$ $1.83(\mathrm{~m}, 4 \mathrm{H}), 2.24(\mathrm{~m}, 2 \mathrm{H}), 2.52-2.62($ complex, $4 \mathrm{H}), 3.70(\mathrm{t}, J=7.0 \mathrm{~Hz}, 2 \mathrm{H}), 4.91(\mathrm{t}, J=$ $4.2 \mathrm{~Hz}, 1 \mathrm{H}) ;{ }^{13} \mathrm{C} \mathrm{NMR}\left(100.6 \mathrm{MHz}, \mathrm{CDCl}_{3}\right) \delta 21.6,21.7,29.4,34.9,38.2,49.4,104.7$, 137.5, 173.4; IR (neat) $1647 \mathrm{~cm}^{-1}$; MS (CI) $\mathrm{m} / z 152\left(\mathrm{M}^{+}+1\right)$; HRMS calcd for $\mathrm{C}_{9} \mathrm{H}_{14} \mathrm{NO}$ $\left(\mathrm{M}^{+}+1\right)$ : 152.1075, found 152.1075. 
Method B. Nitrone 32 (50 mg, $0.33 \mathrm{mmol})$, using method B, afforded $5 \mathrm{mg}$ $(10 \%)$ lactam 34 as an oil.

\section{References:}

(1) Smith, B. T.; Wendt, J. A.; Aubé, J. Org. Lett. 2002, 4, 2577-2579.

(2) Milligan, G. L.; Mossman, C. J.; Aubé, J. J. Am. Chem. Soc. 1995, 117, 10449-59.

(3) Wrobleski, A.; Aubé, J. J. Org. Chem. 2001, 66, 886-889.

(4) Staszak, M. A.; Doecke, C. W. Tetrahedron Lett. 1993, 34, 7043-4.

(5) Wendt, J. A.; Aubé, J. Tetrahedron Lett. 1996, 37, 1531-4. 

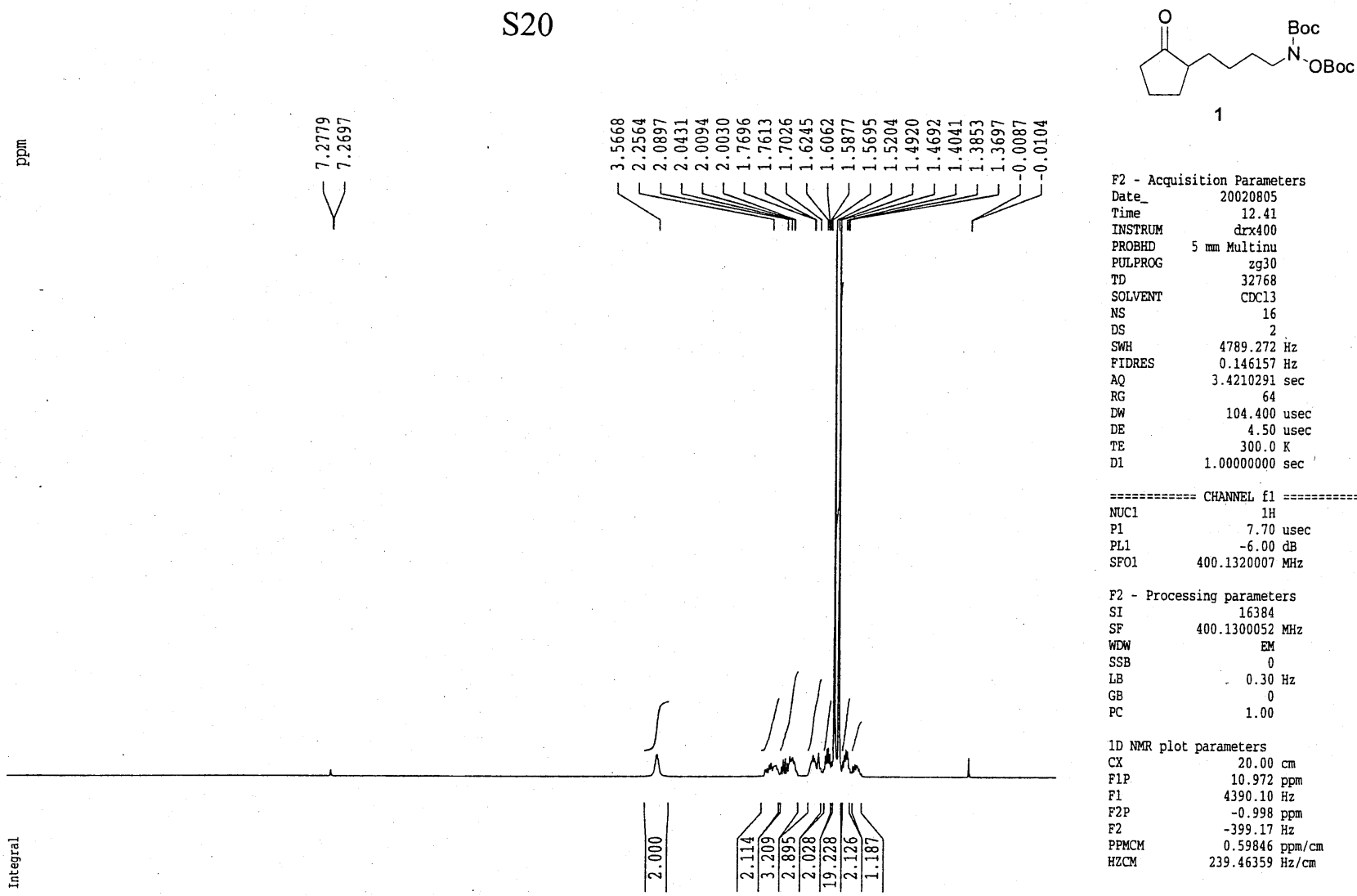

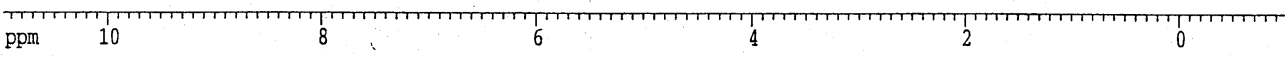

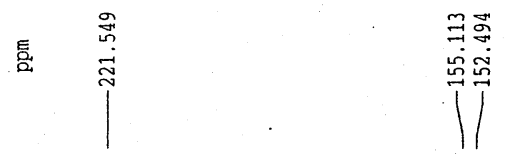

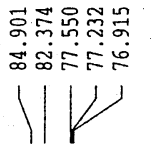
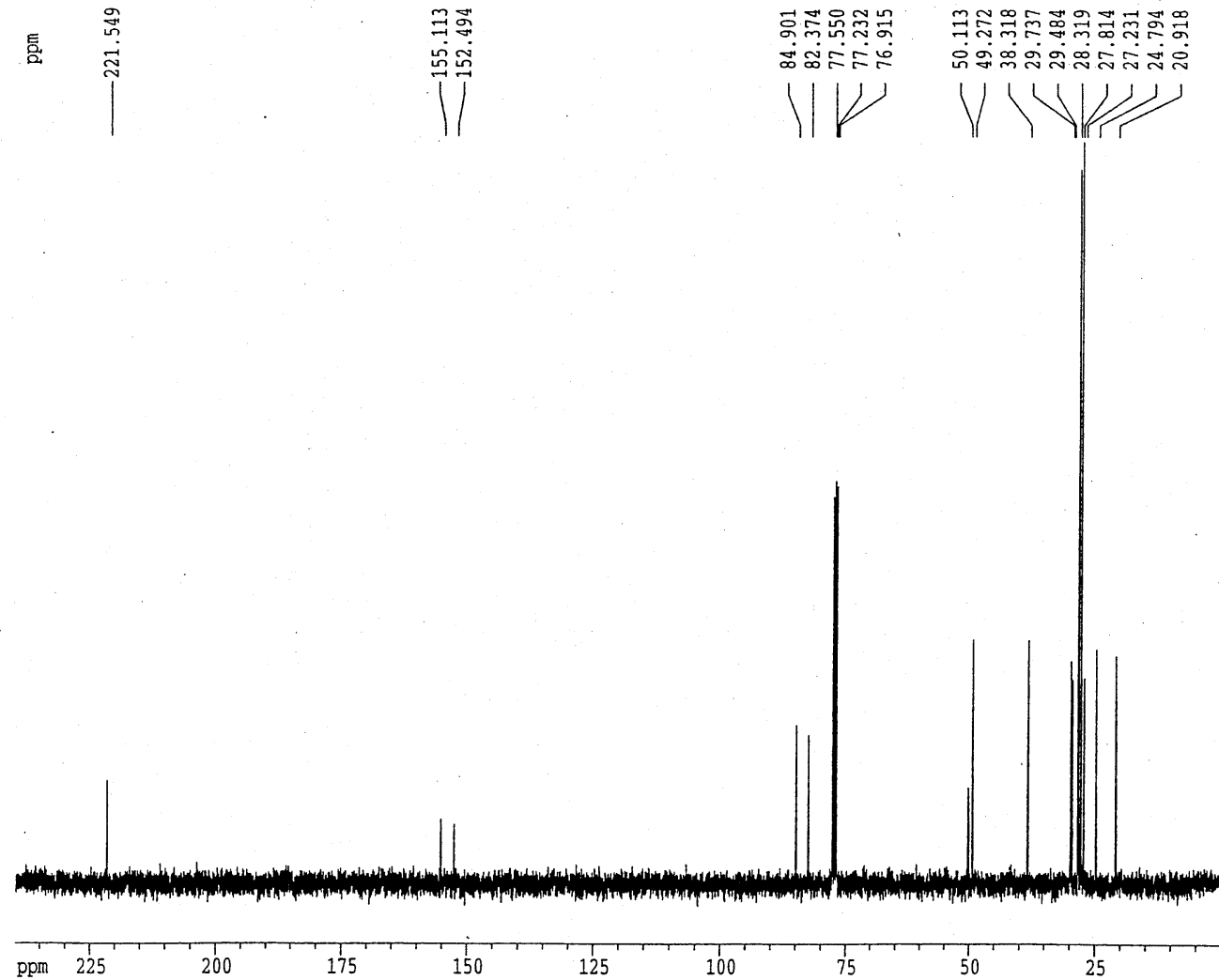

175

150

125

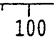

75

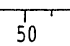

25

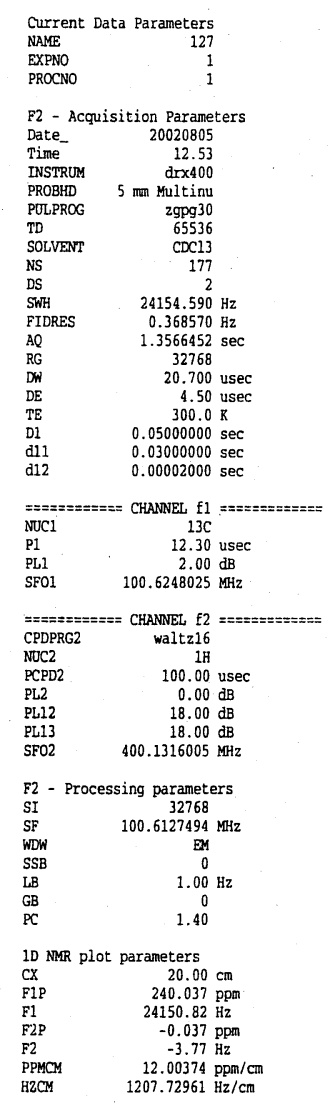



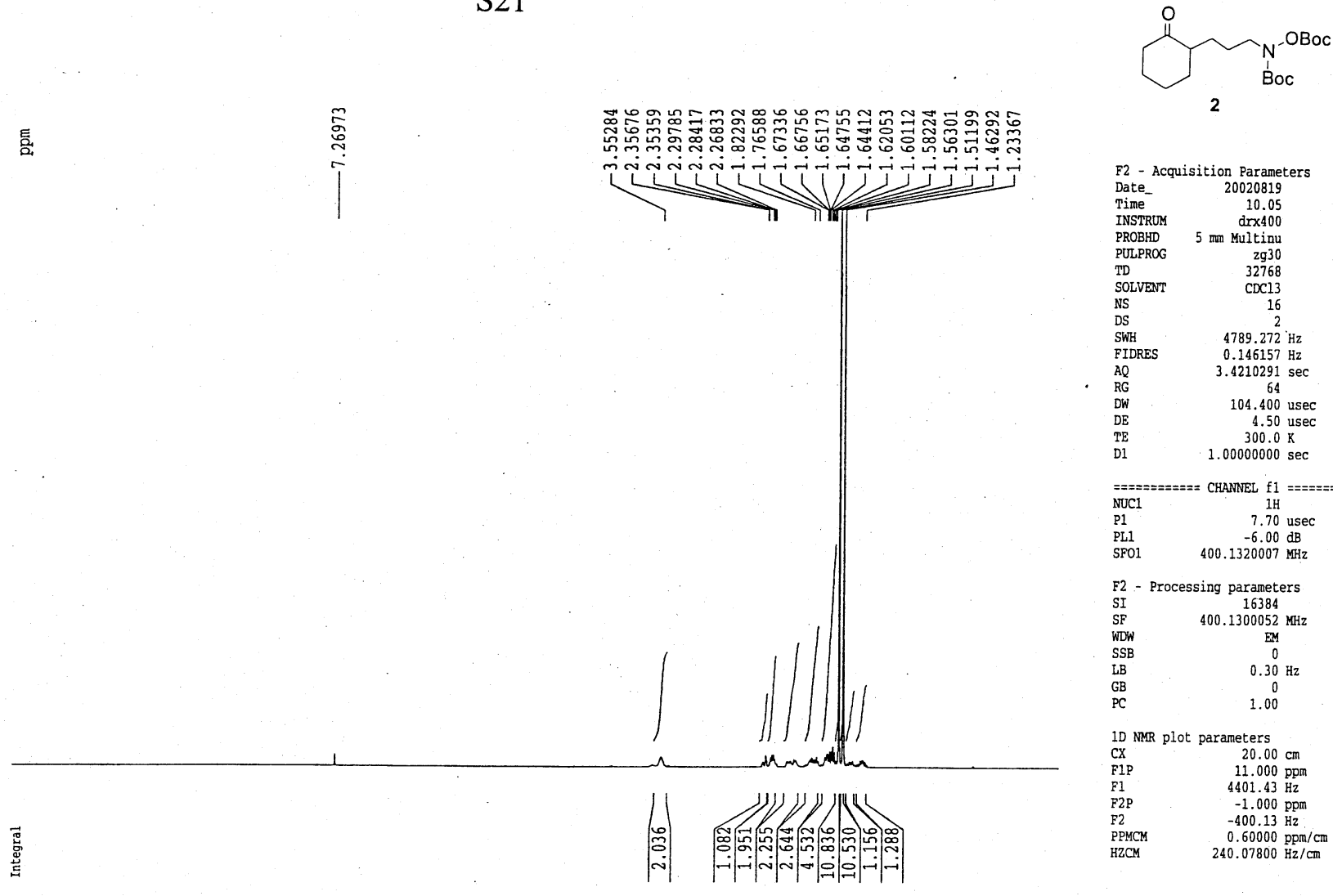

2
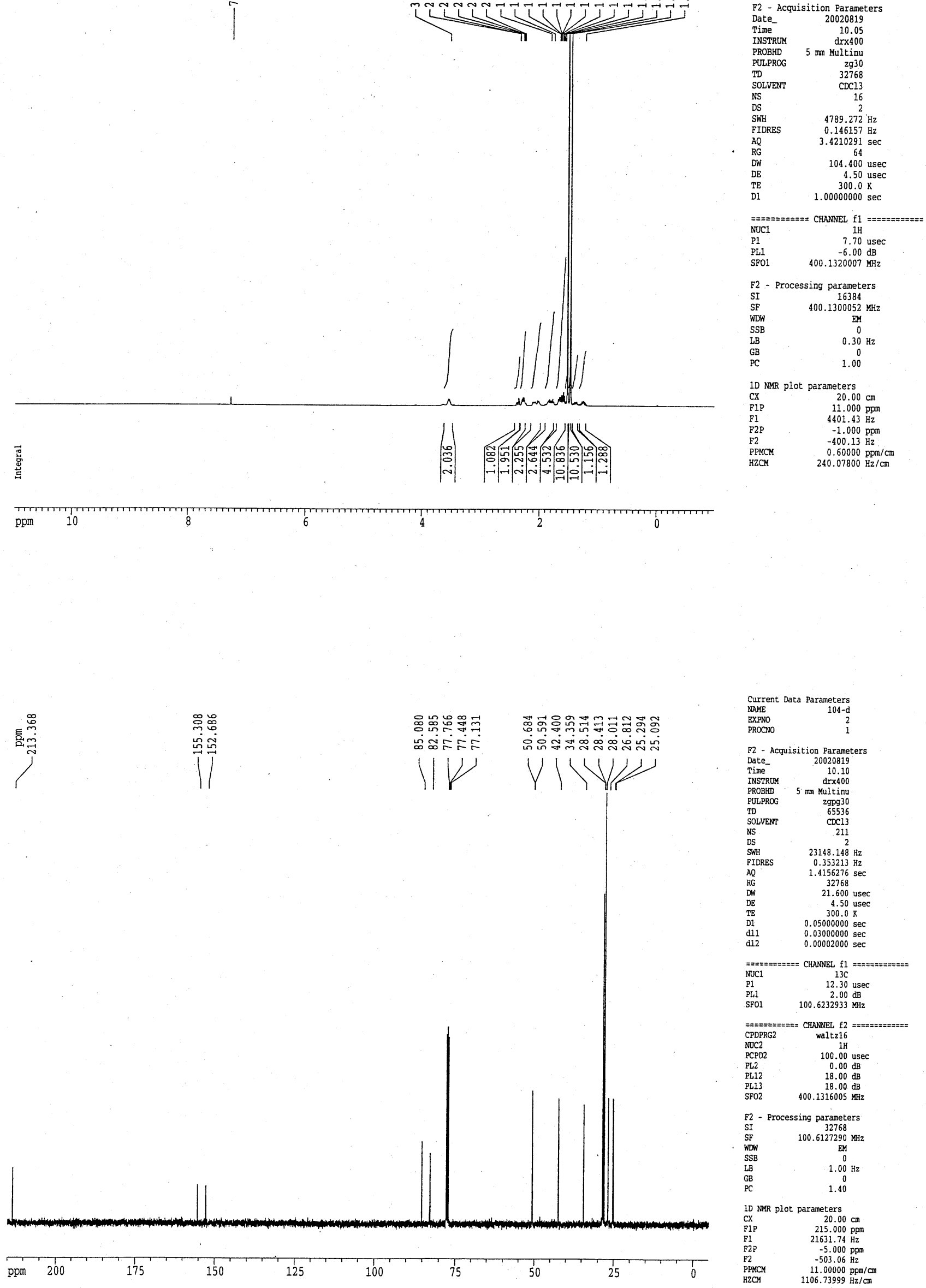

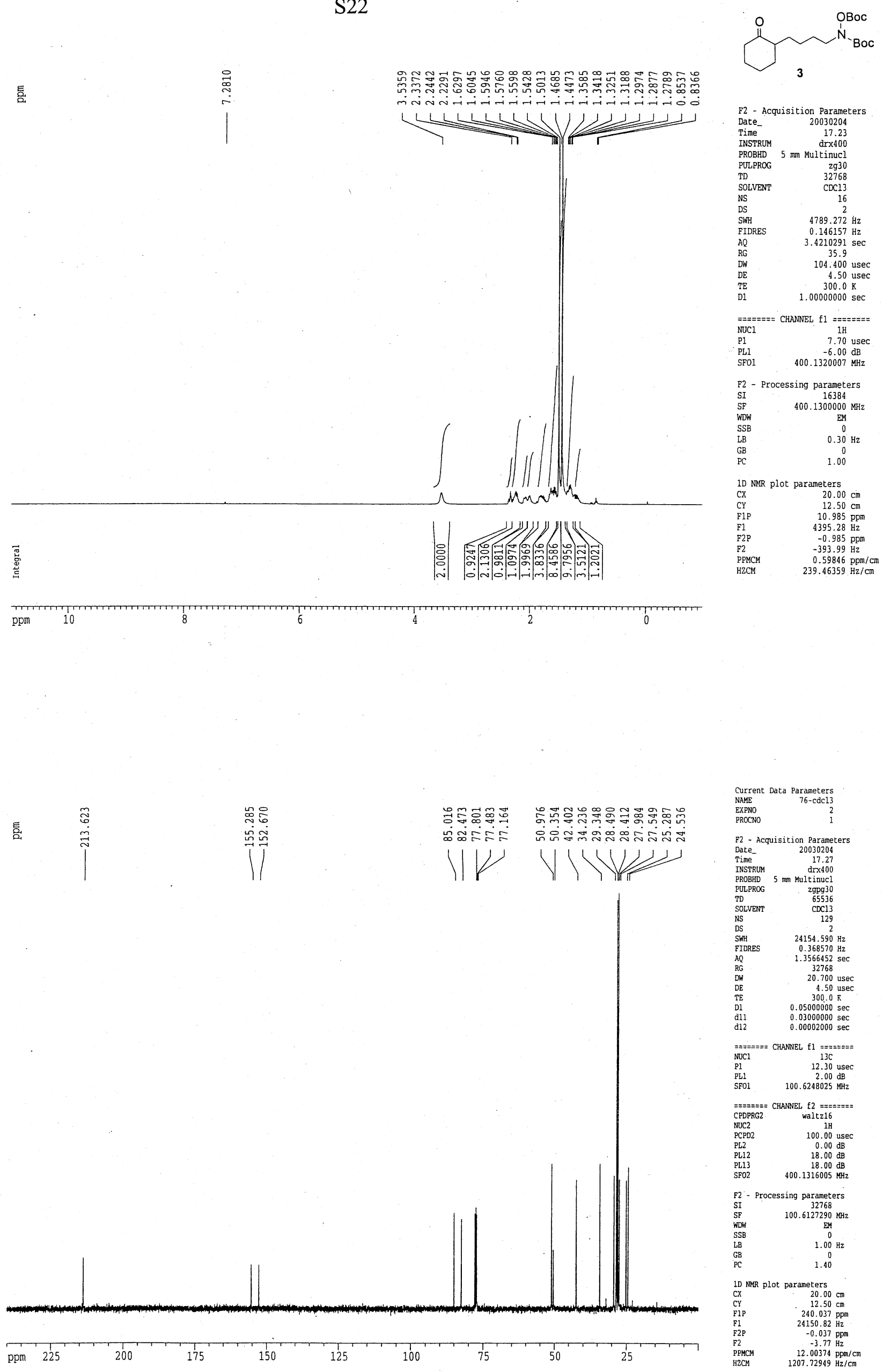

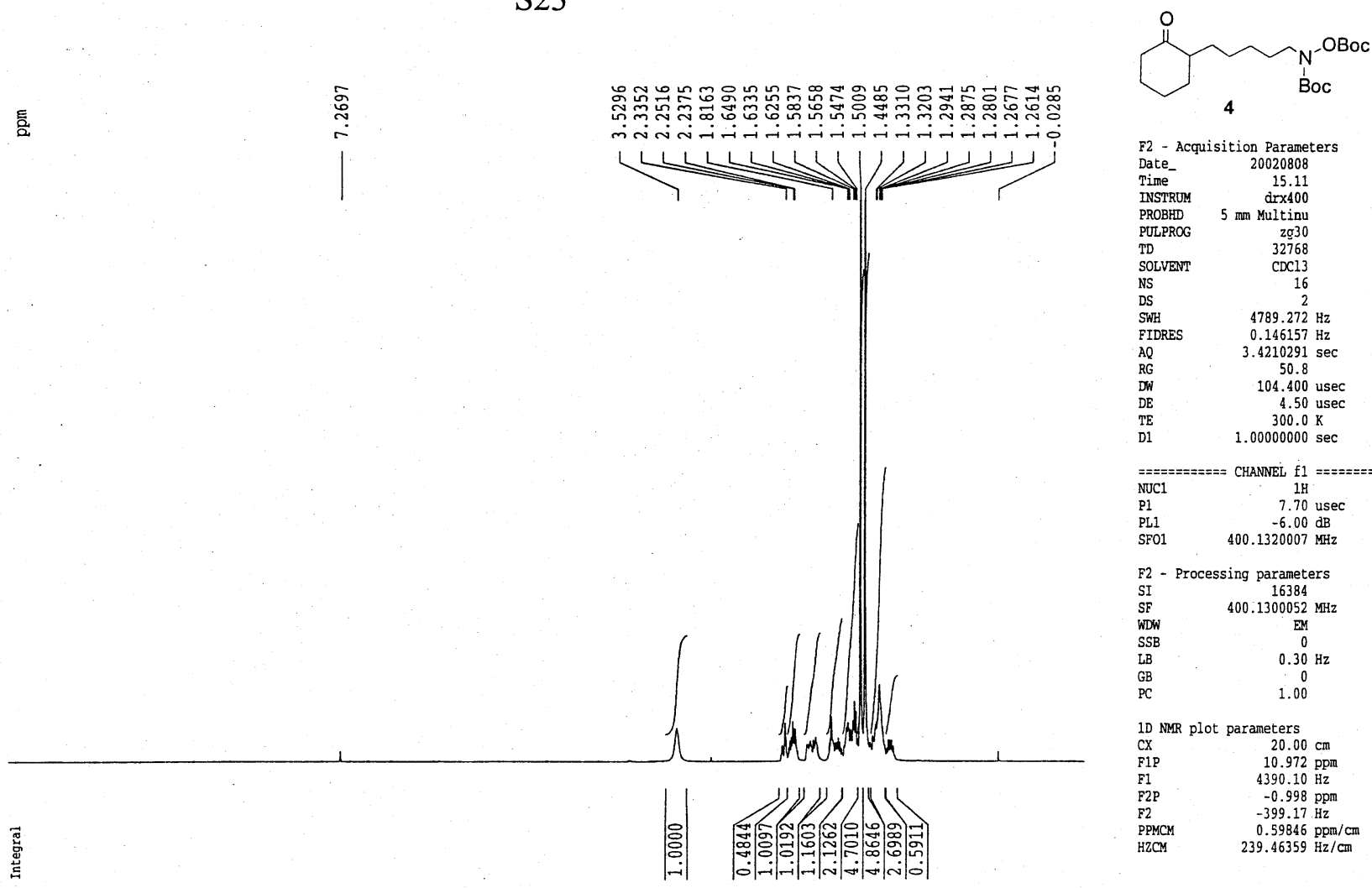

$========= \pm 2$ CHANNEL f1 =z=s=:=z=:=

$\begin{array}{lll}\mathrm{NUC1} & 1 \mathrm{H} \\ \mathrm{P1} & 7.70 \mathrm{usec}\end{array}$ $\begin{array}{ll}\text { PL1 } & -6.00 \mathrm{~dB} \\ \text { SF01 } & 400.1320007 \mathrm{MHz}\end{array}$

F2 - Processing parameters

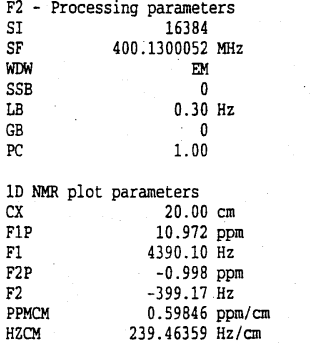

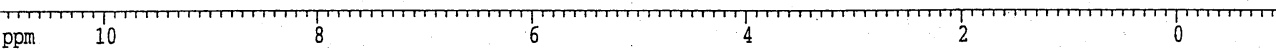

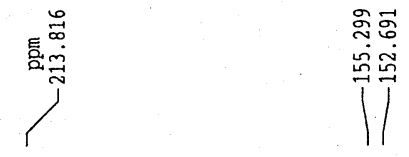

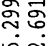

1)

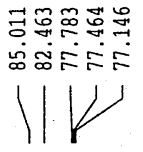

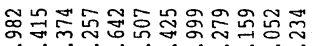

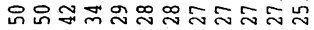

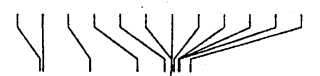

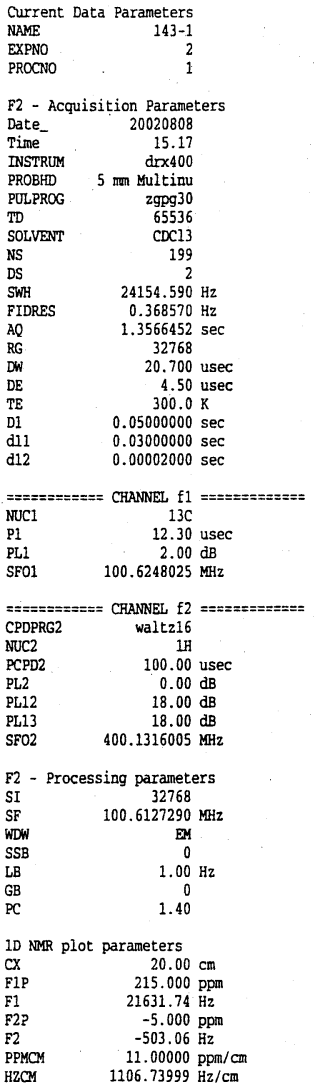



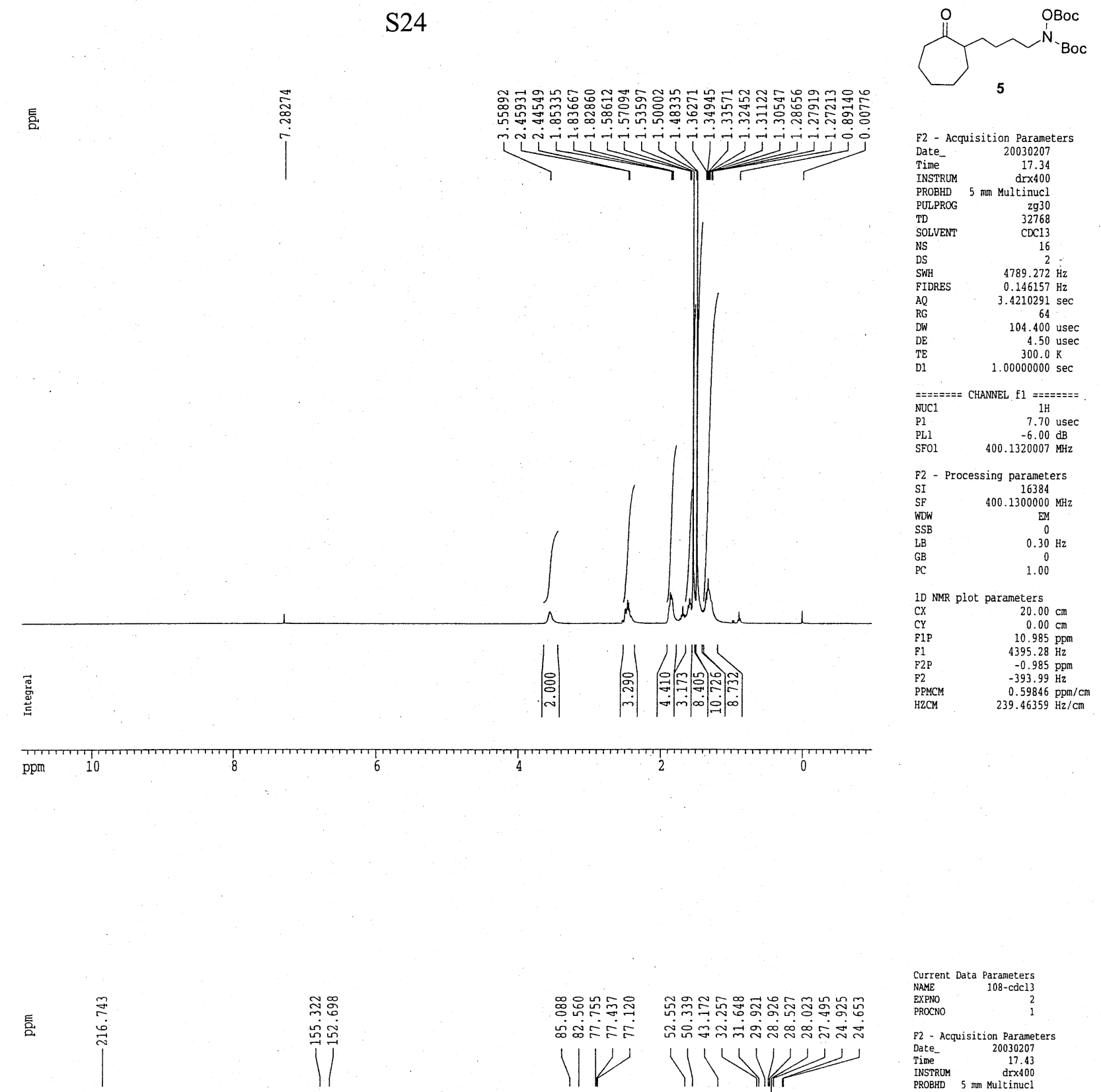

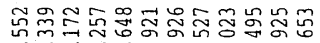
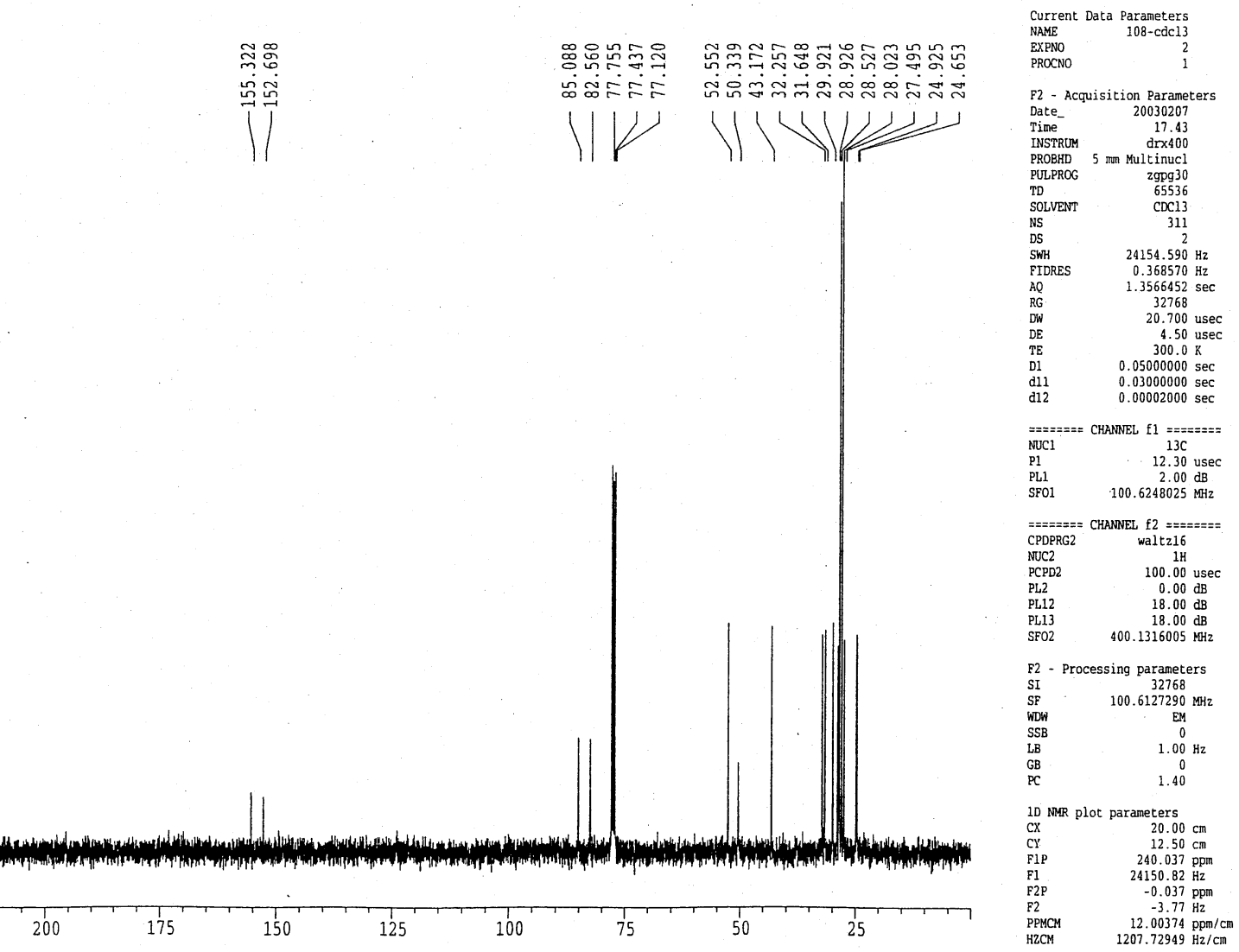

200

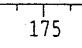

150

12

125

100

75

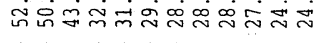
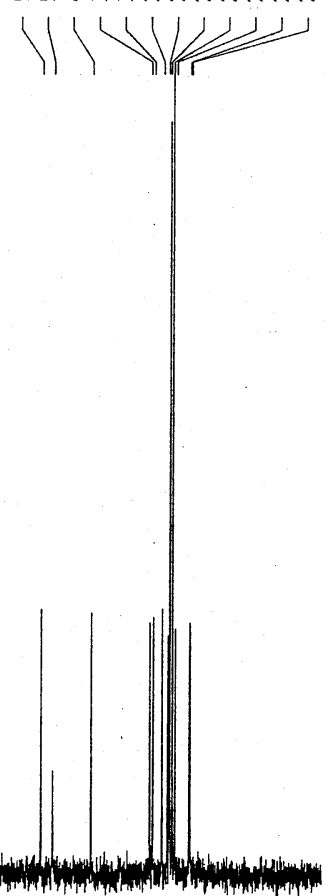

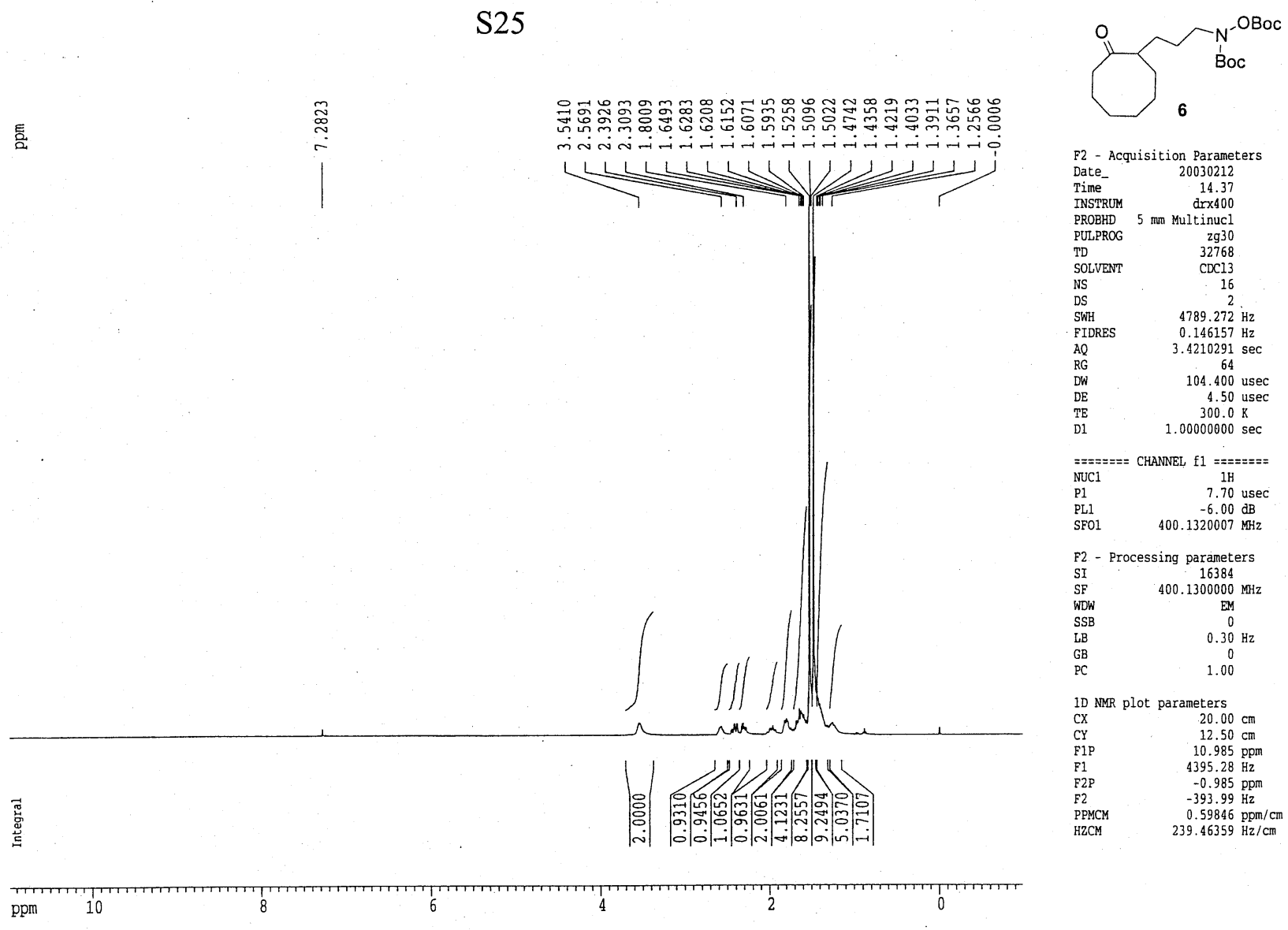

F2 - Processing parameters

SI Processing parameters

$\begin{array}{ll}\text { SF } & 400.1300000 \mathrm{MHz}\end{array}$

$\begin{array}{lc}\text { WDW } & \text { EM } \\ \text { SSB } & 0 \\ \text { LB } & 0.30 \mathrm{~Hz}\end{array}$

$\begin{array}{lr}\mathrm{GB} & 0 \\ \mathrm{PC} & 1.00\end{array}$

1D NMR plot parameters

$\begin{array}{lc}\text { CX } & 20.00 \mathrm{~cm} \\ \text { CY } & 12.50 \mathrm{~cm} \\ \text { F1P } & 10.985 \mathrm{ppm} \\ \text { F1 } & 4395.28 \mathrm{~Hz} \\ \text { F2P } & -0.985 \mathrm{ppm} \\ \text { F2 } & -393.99 \mathrm{~Hz} \\ \text { PPMCM } & 0.59846 \mathrm{ppm} / \mathrm{cm} \\ \text { HZCM } & 239.46359 \mathrm{~Hz} / \mathrm{cm}\end{array}$

ppm

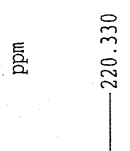

|

웍성웡웍

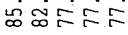

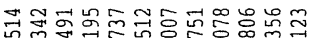

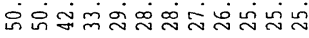

11
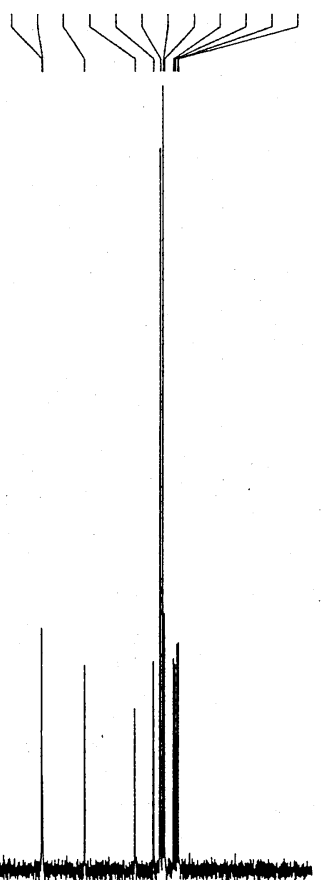

ppm 225

20

200

175

125

100

75

50

50

25

$\frac{1}{25}$



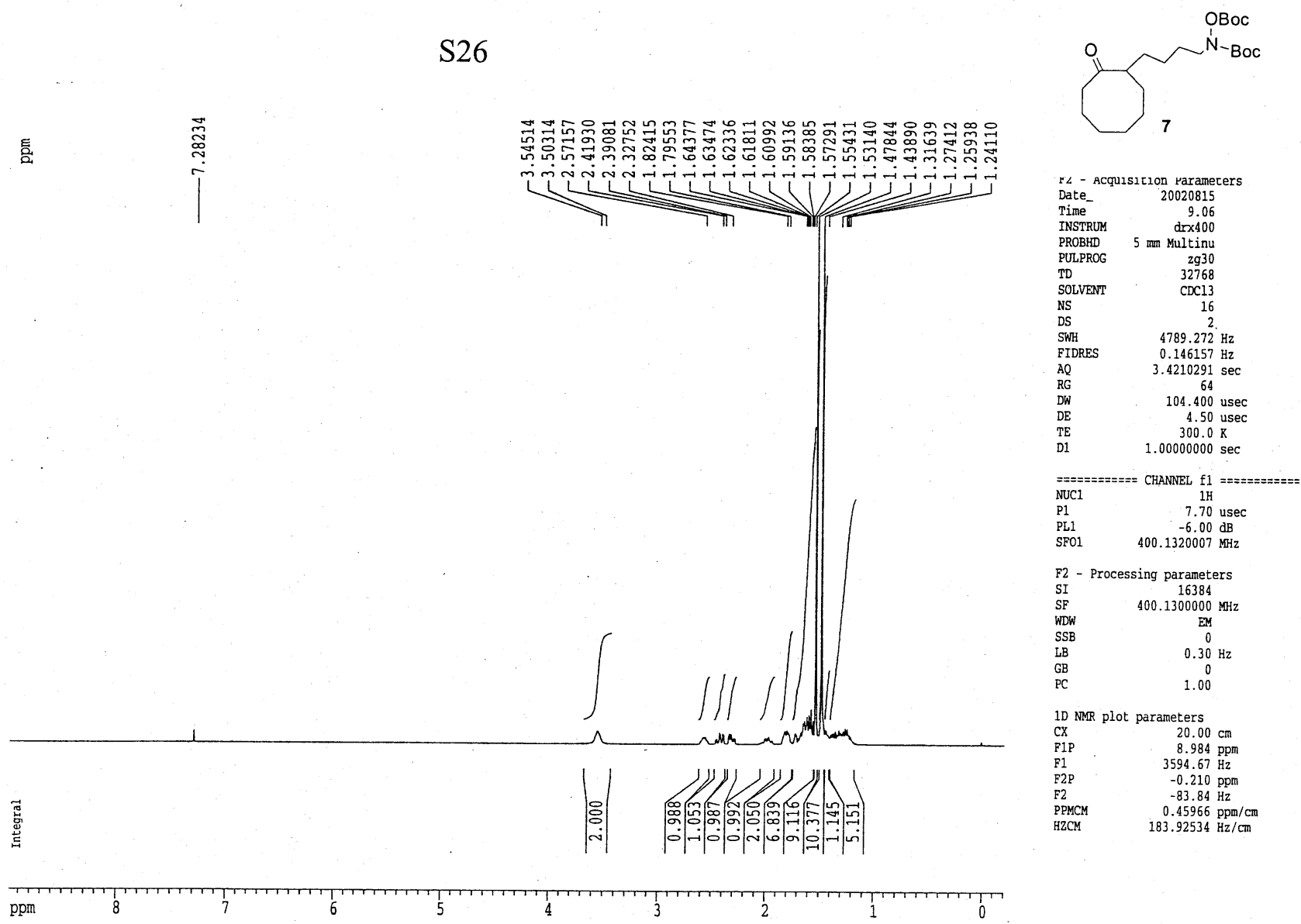

ppm

c)

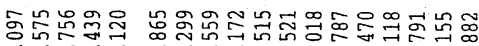

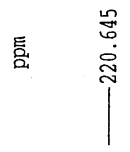

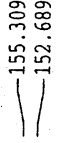

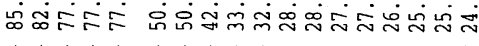

11113

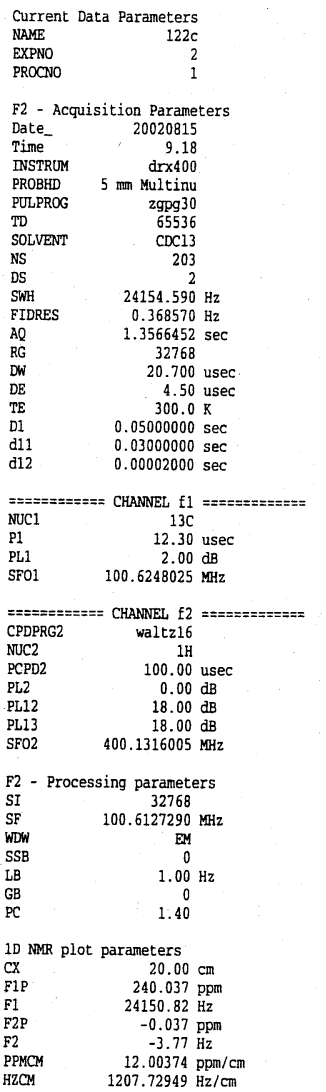



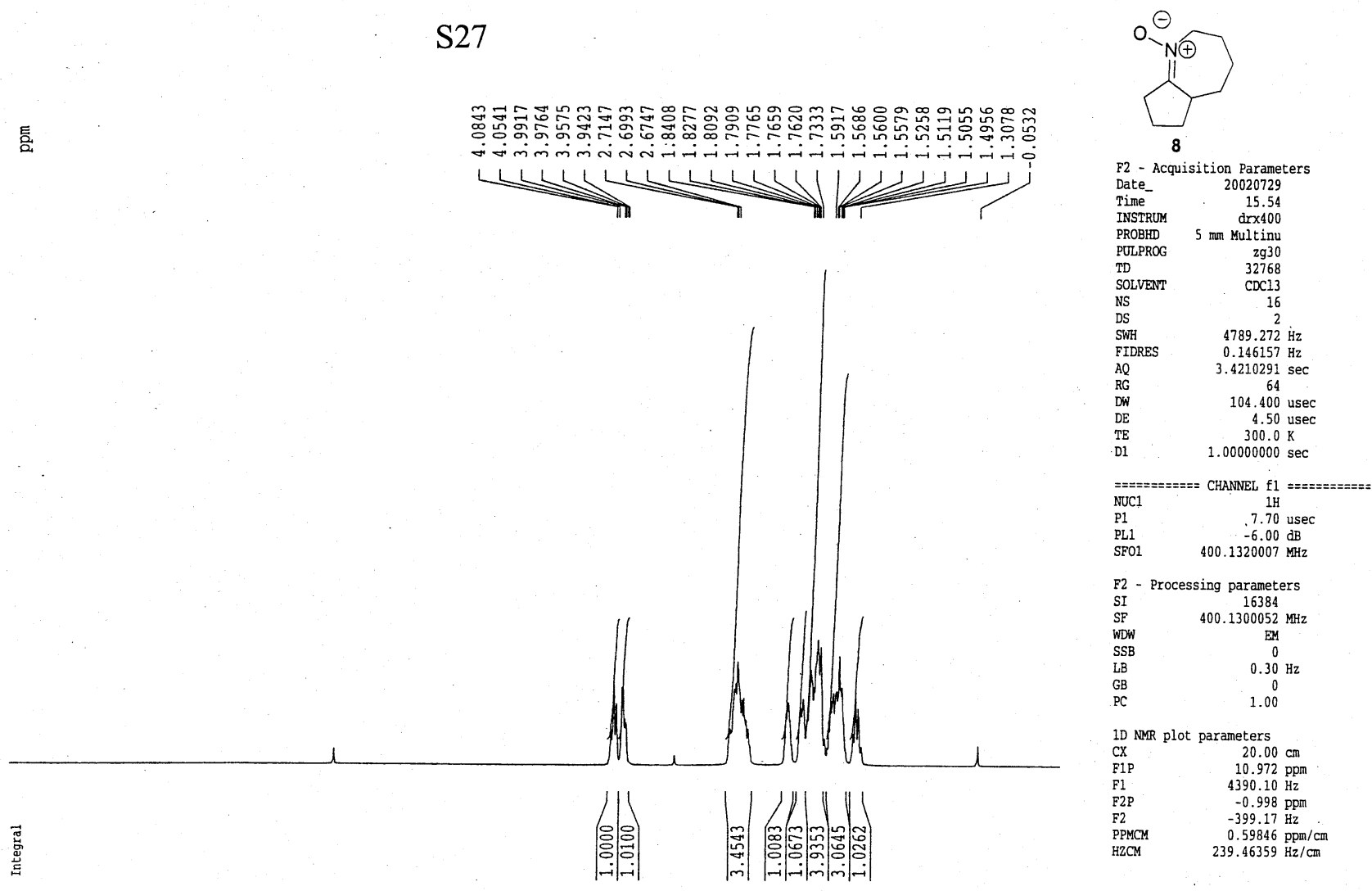

pp

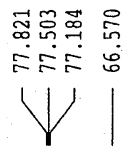

镸

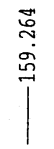

ind

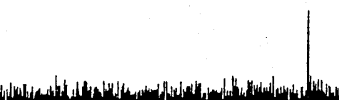

$\mid$

ppm 200

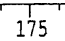

150

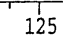

100

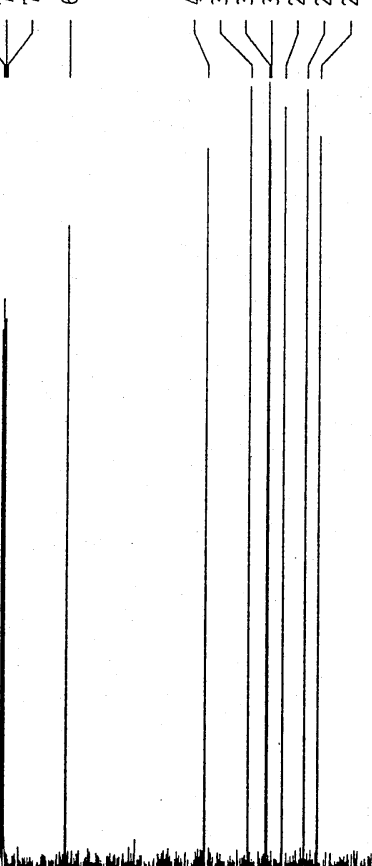

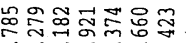
ษ

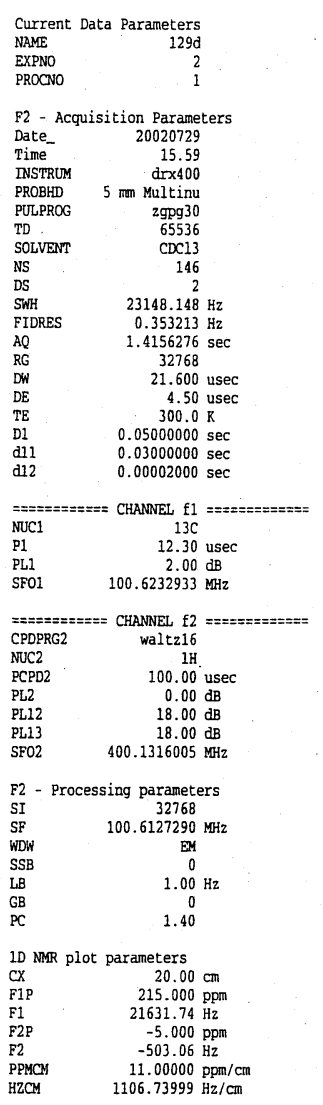



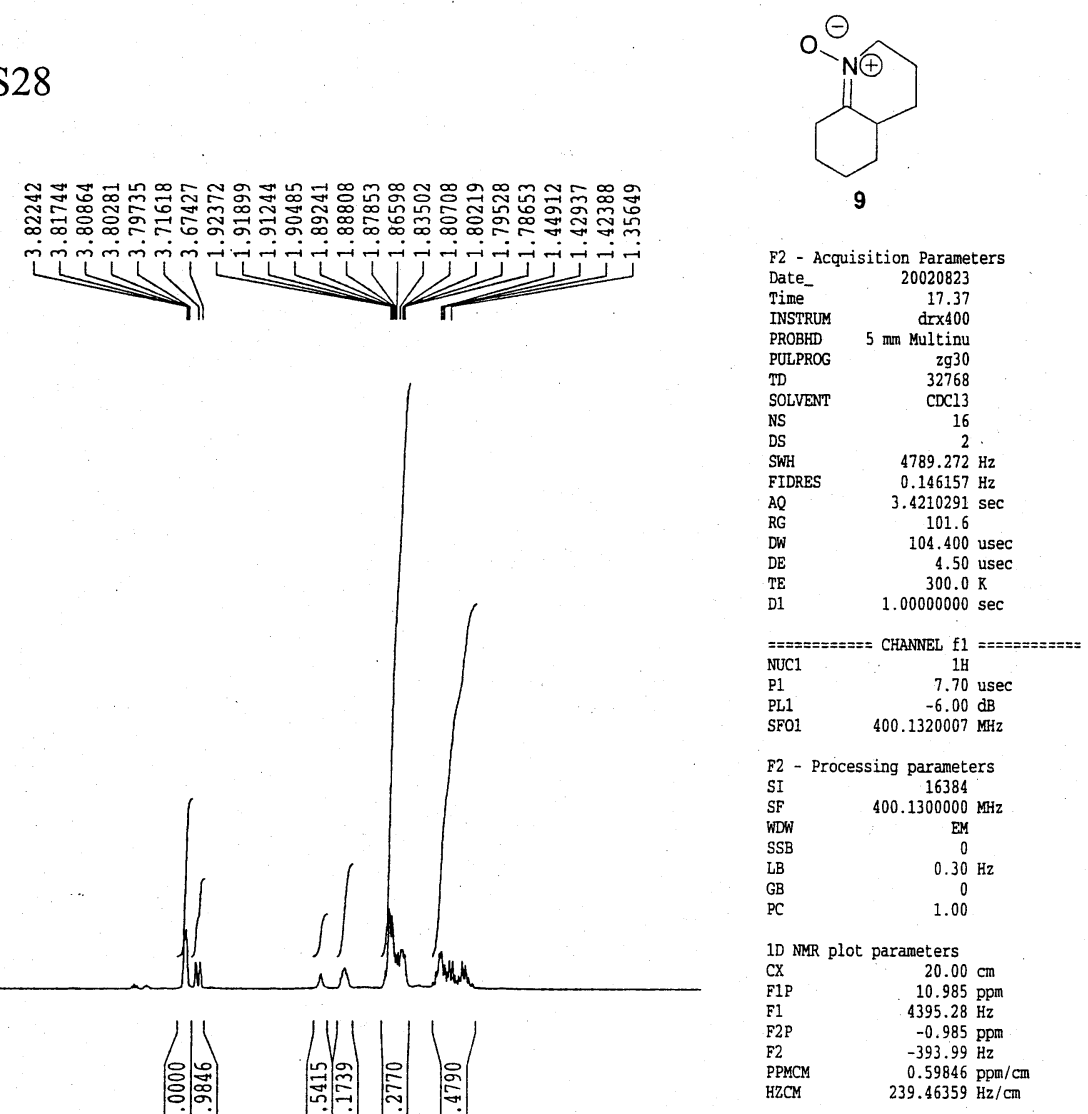

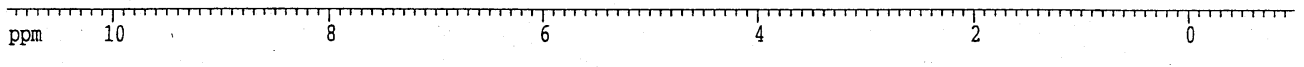

镸

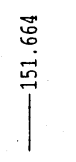

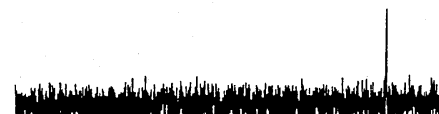

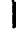
(npm

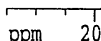

ppm 200
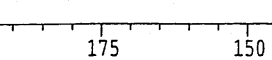

150

$1,1,100$




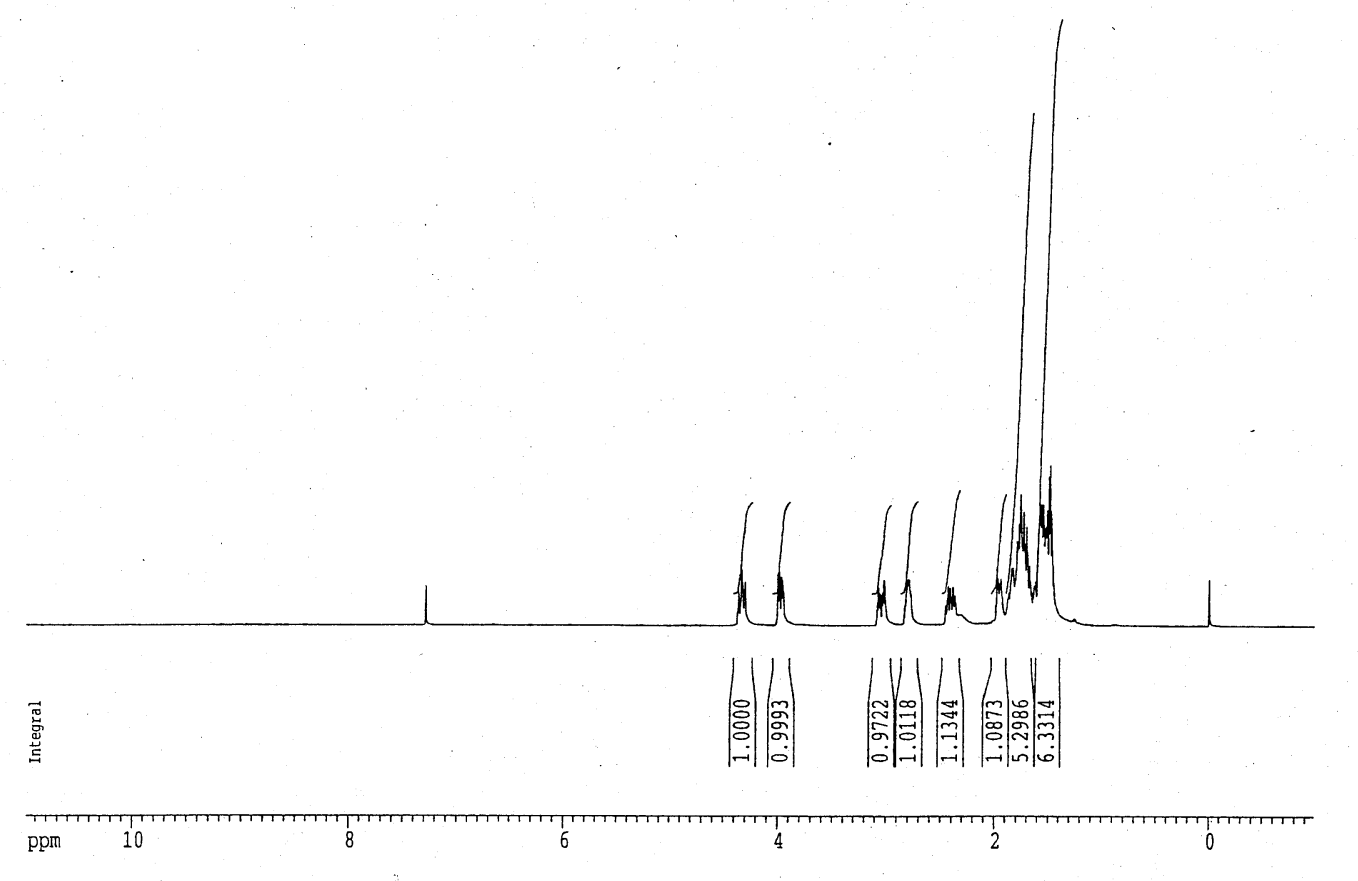

\section{홍}

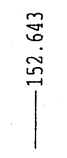

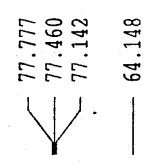

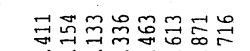

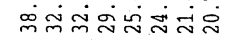

UIII
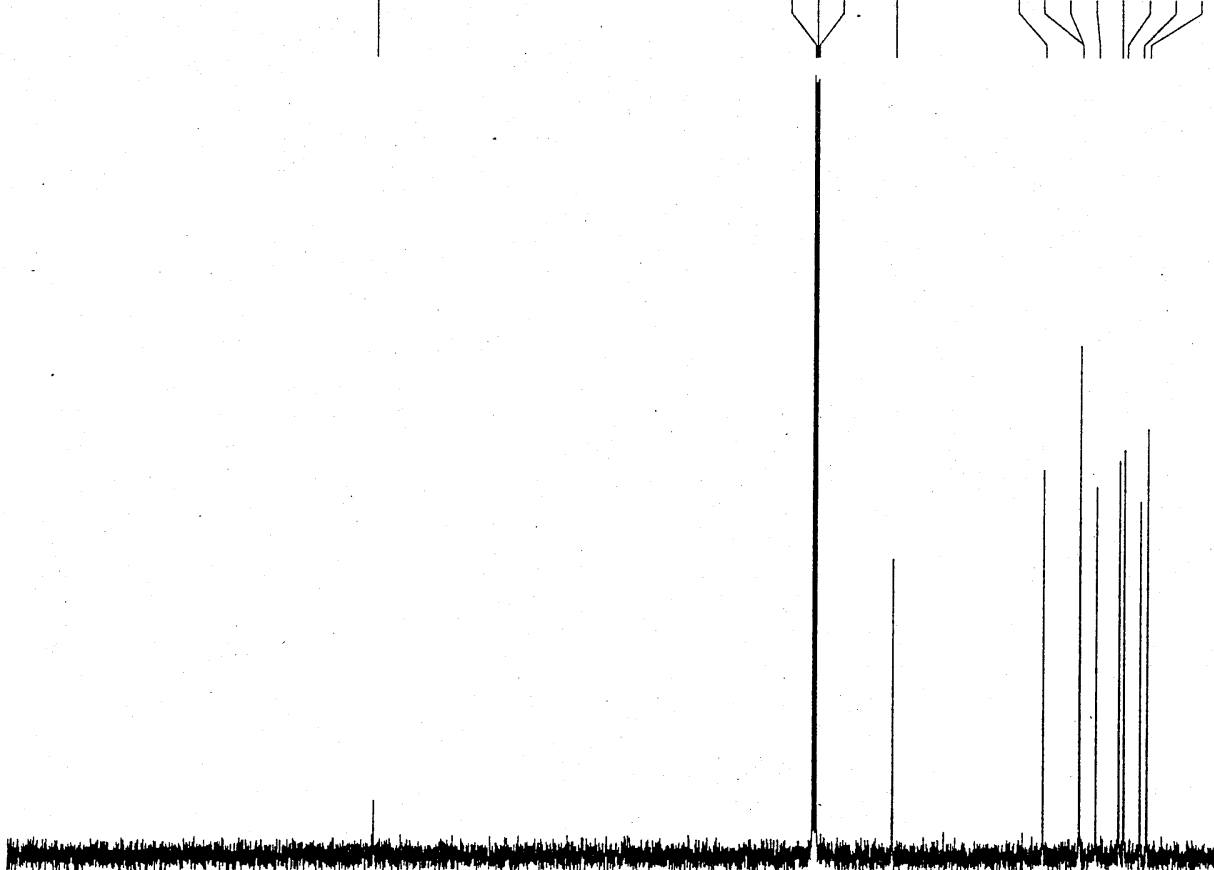

ppm

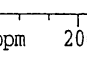

200

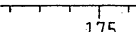

175

150

125

100

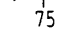

50

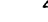

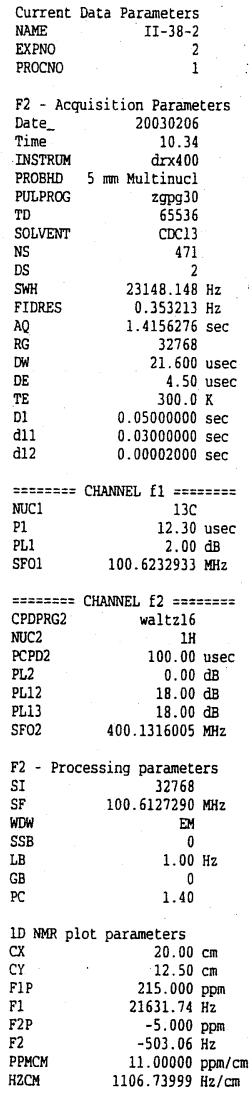




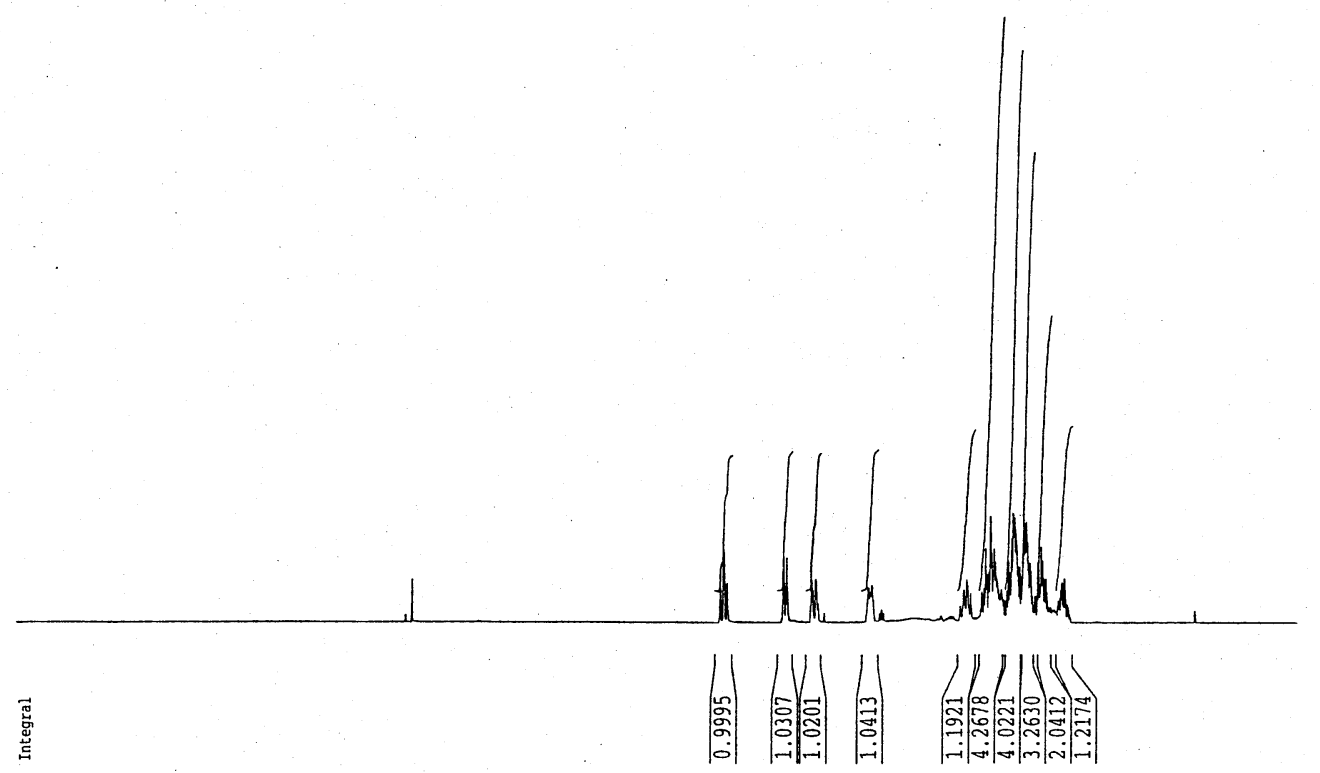

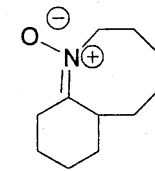

11

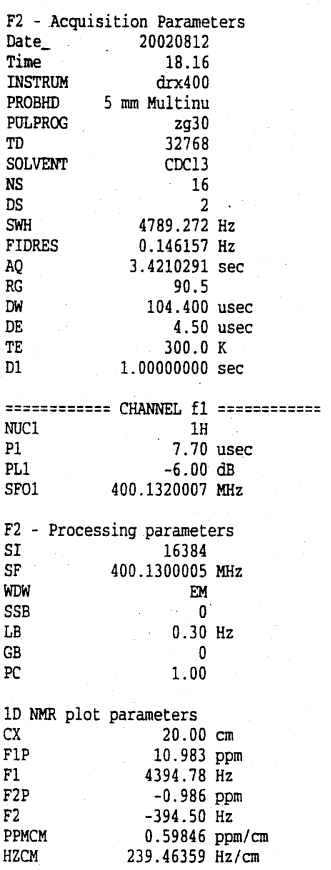

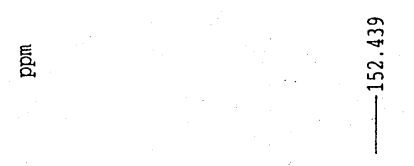

175

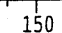

오ำ

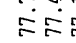

V

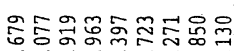

ம்

1112 


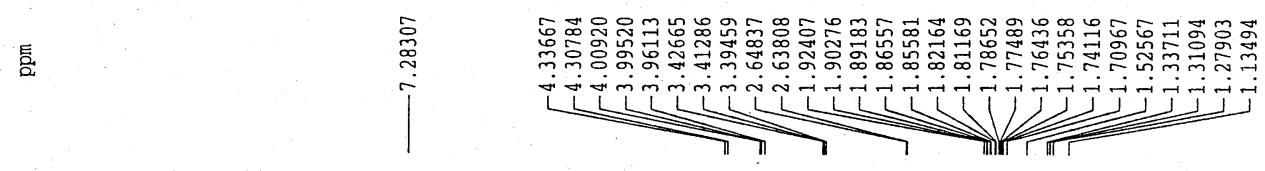

12

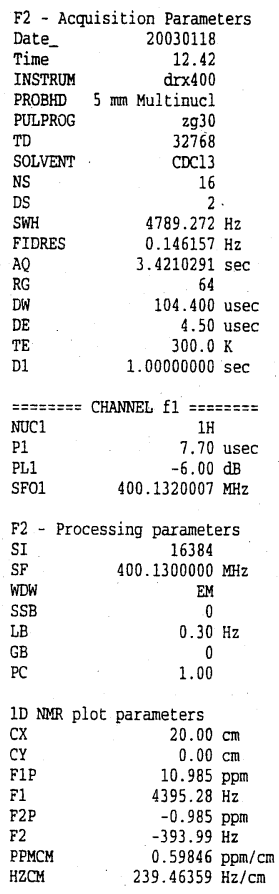

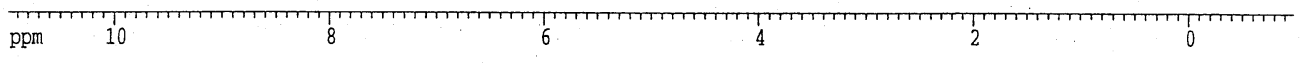
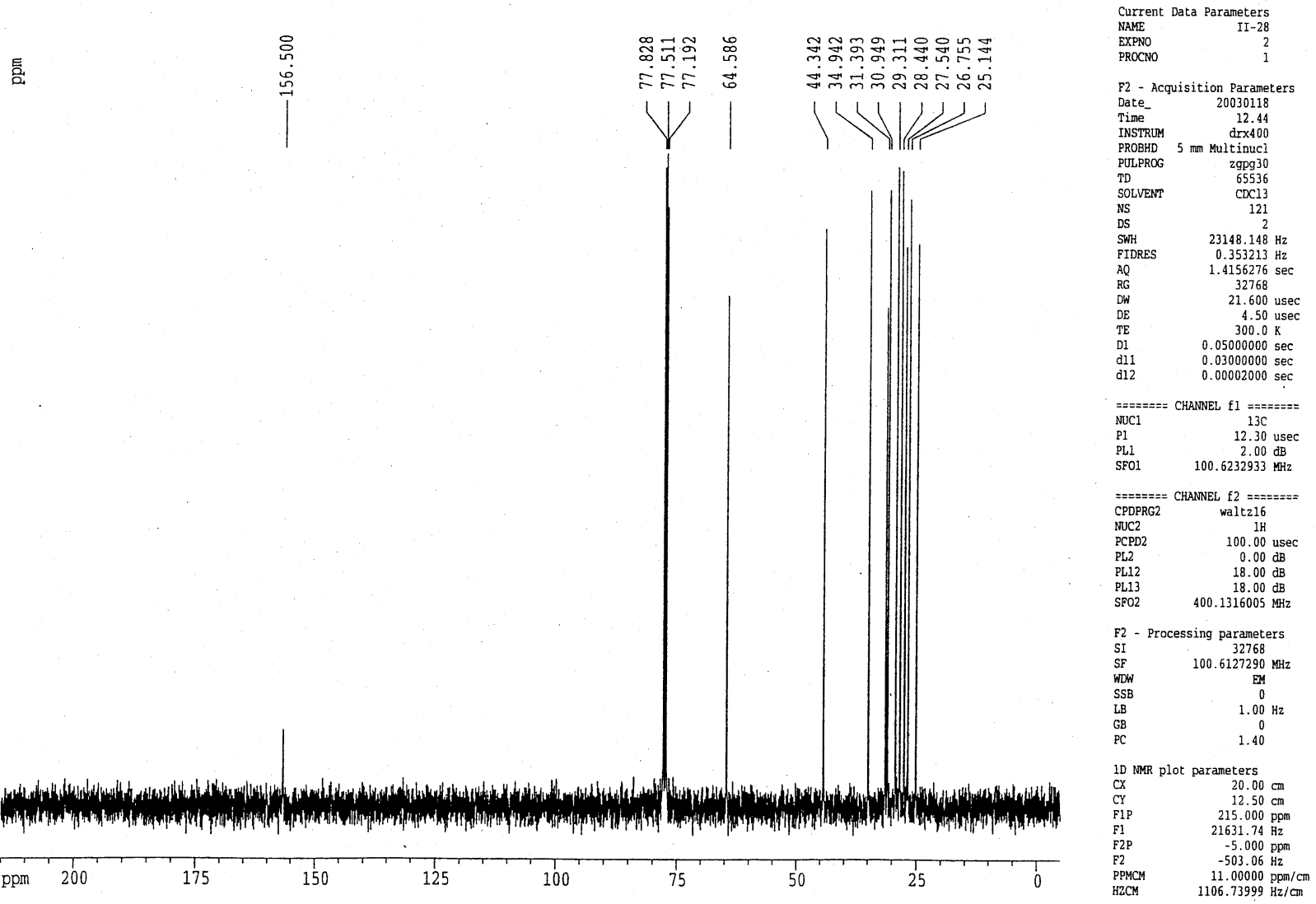
S32
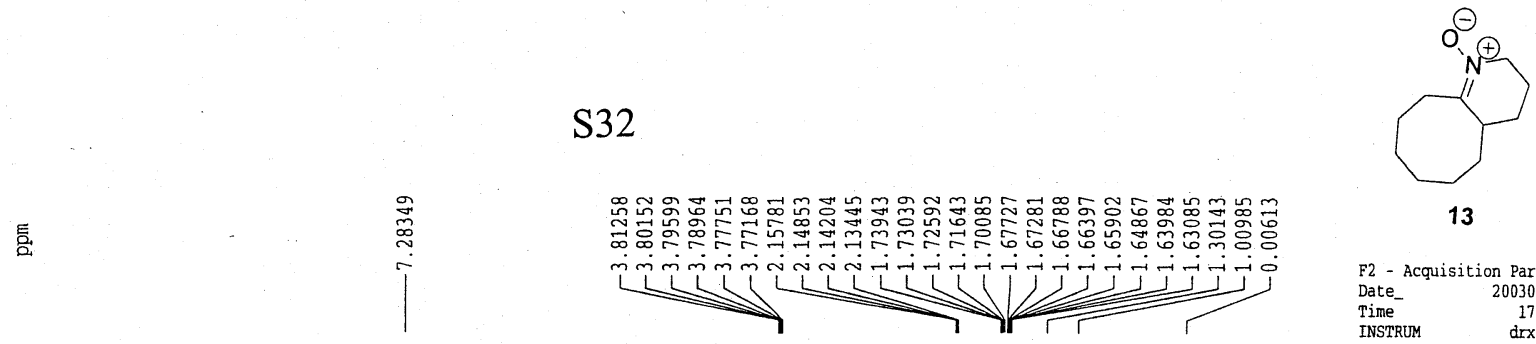

13

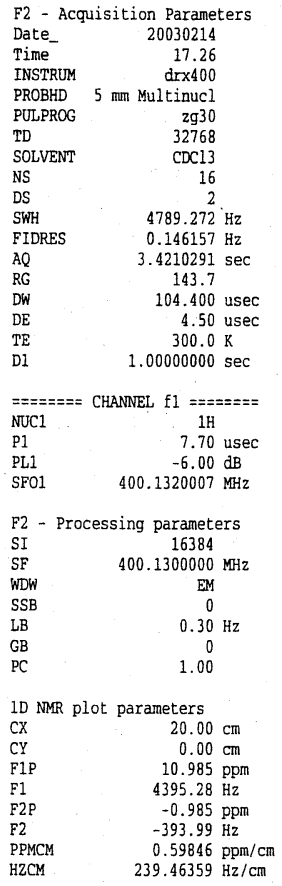

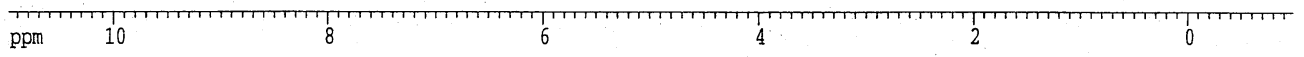

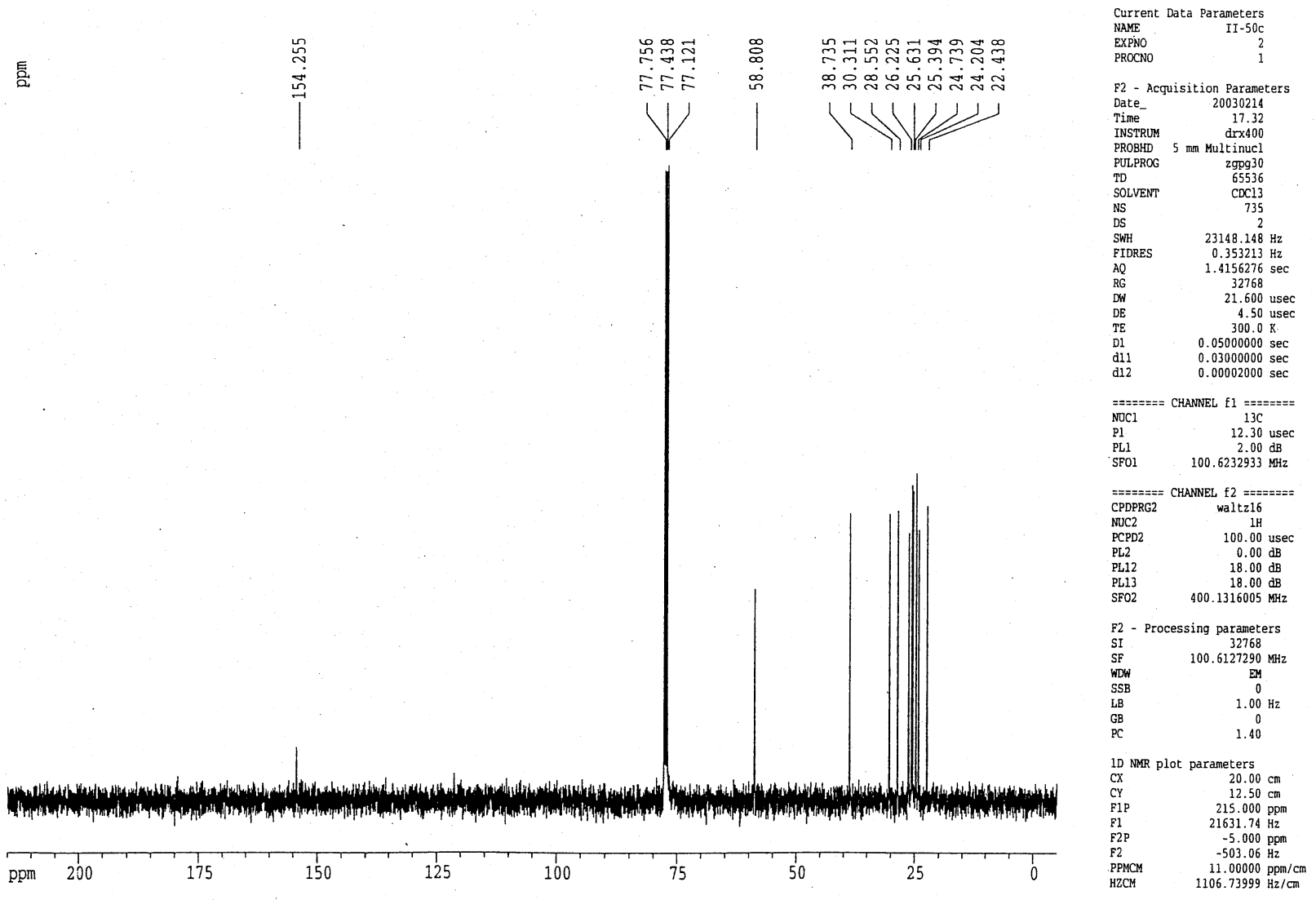



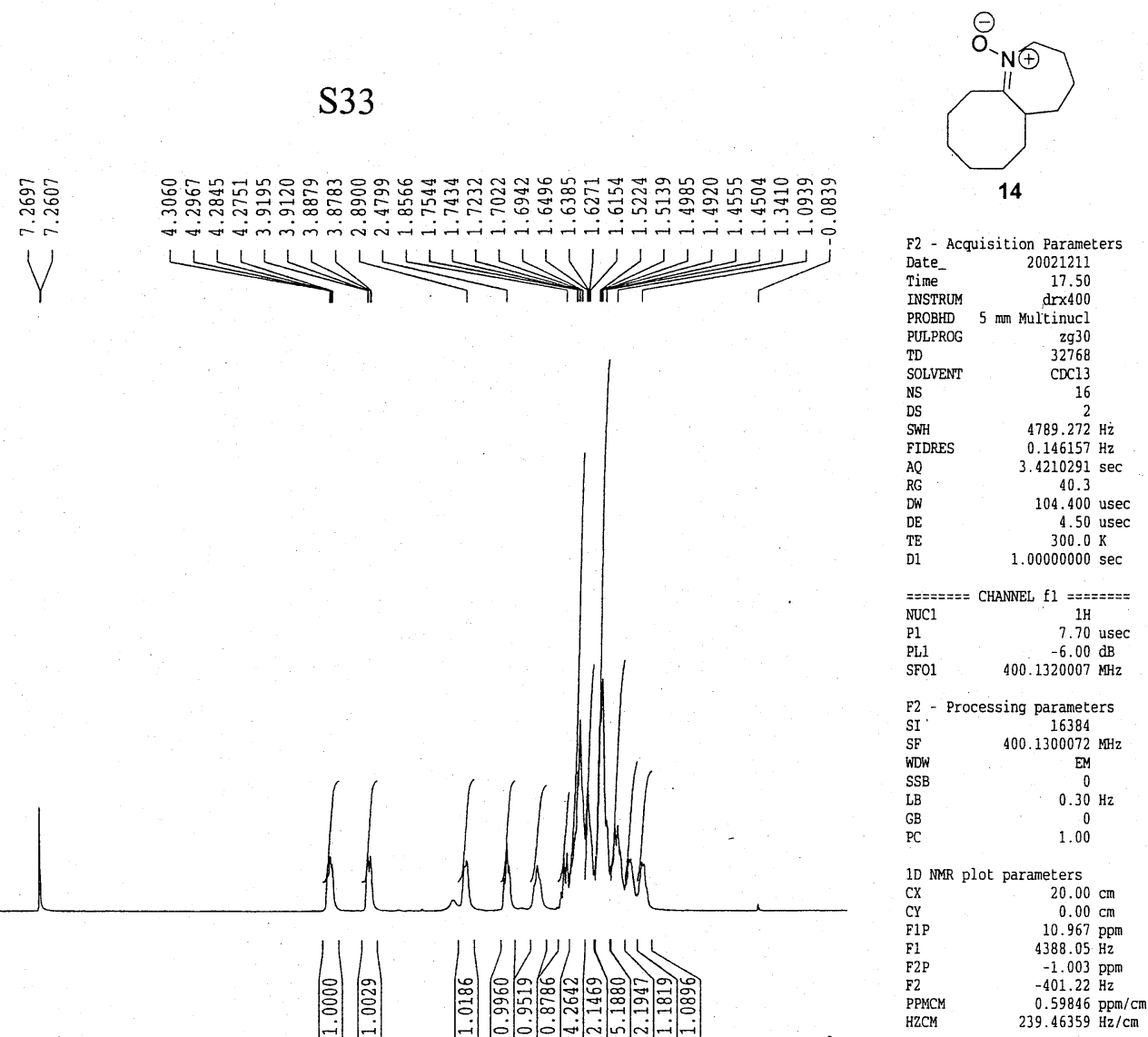

$\overrightarrow{\widetilde{J}}$
离
吕
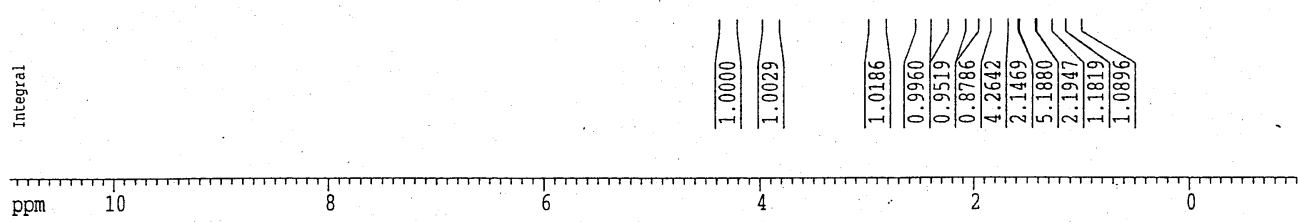

镸

\section{Бू}

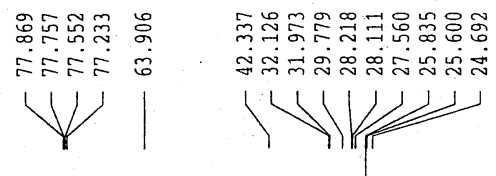

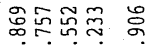

तहล

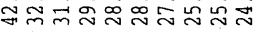

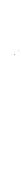




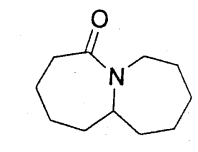

18

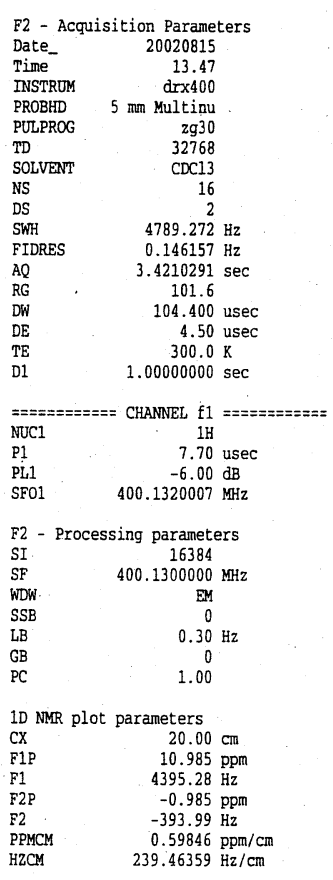

\section{1}
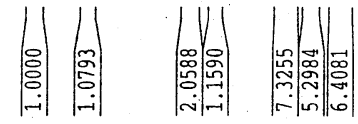

ppm 10
ppm 10

镸

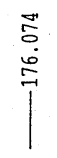

눙웡워

도유

$Y$

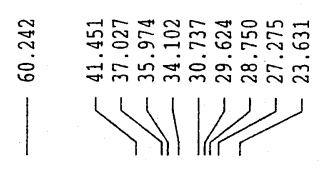

रा111113

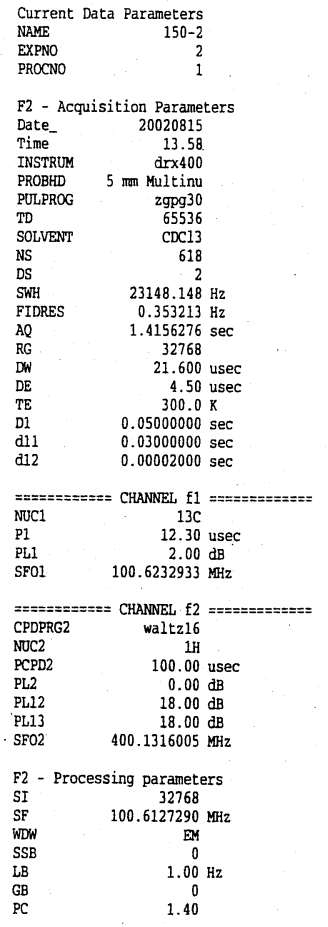

ID NMR plot parameters

CX $20.00 \mathrm{~cm}$ $215.000 \mathrm{ppm}$
$21631.74 \mathrm{~Hz}$ $-5.000 \mathrm{ppm}$ $-503.06 \mathrm{~Hz}$ $11.00000 \mathrm{ppm} / \mathrm{cm}$ 

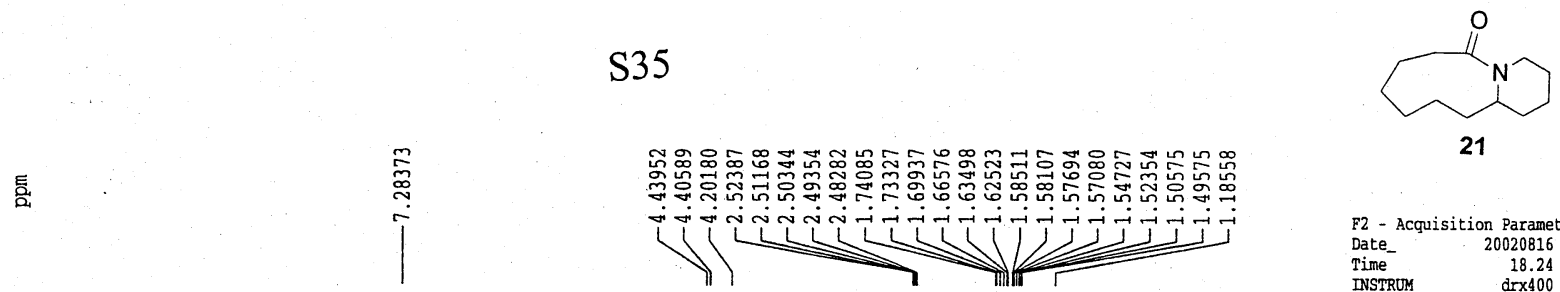

21
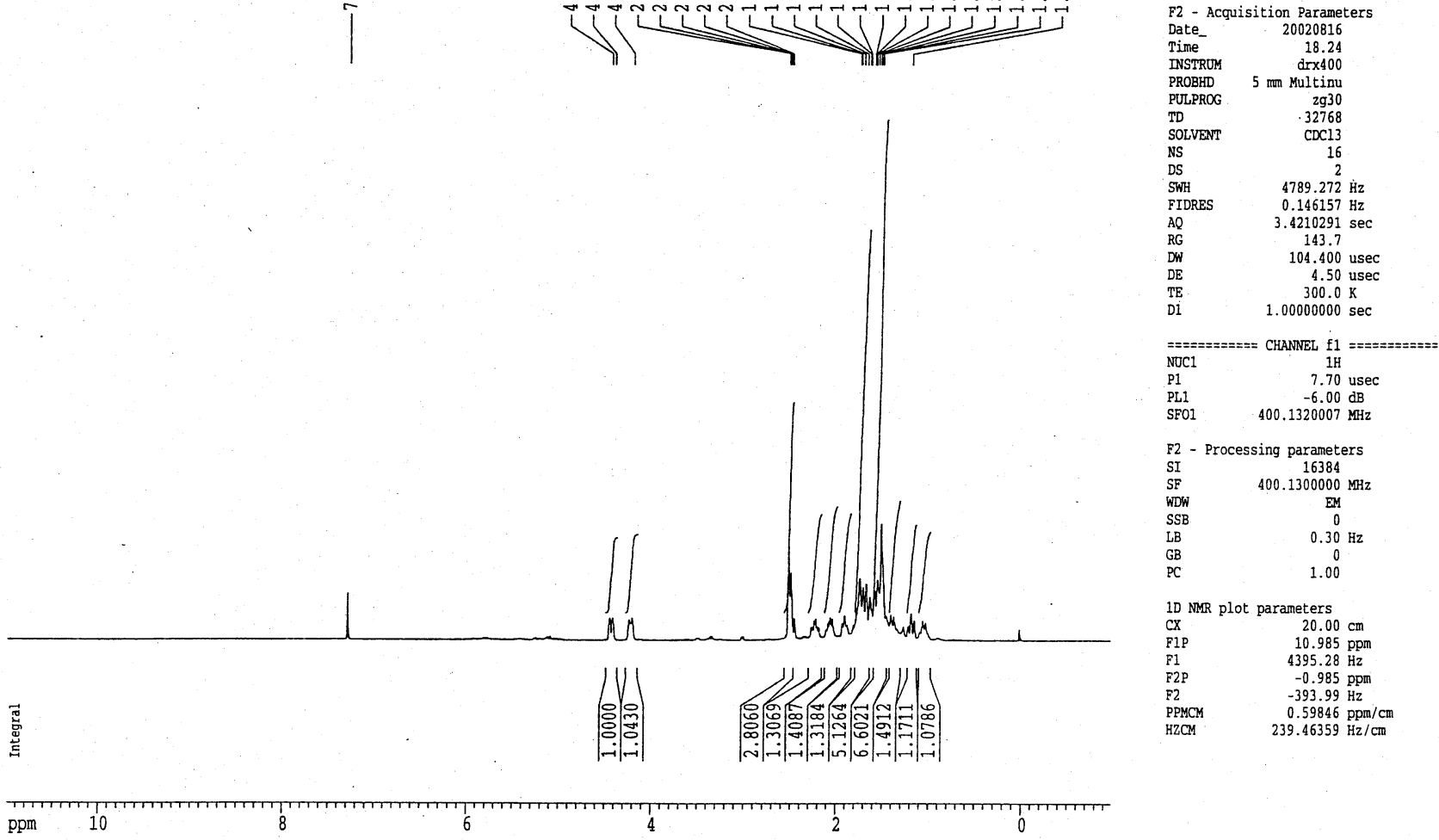

$$
\text { pp }
$$

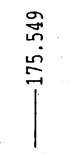

镸
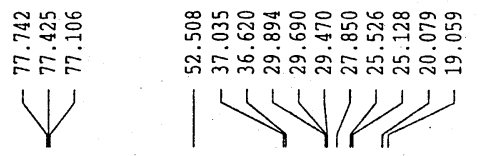

중 궝유

11

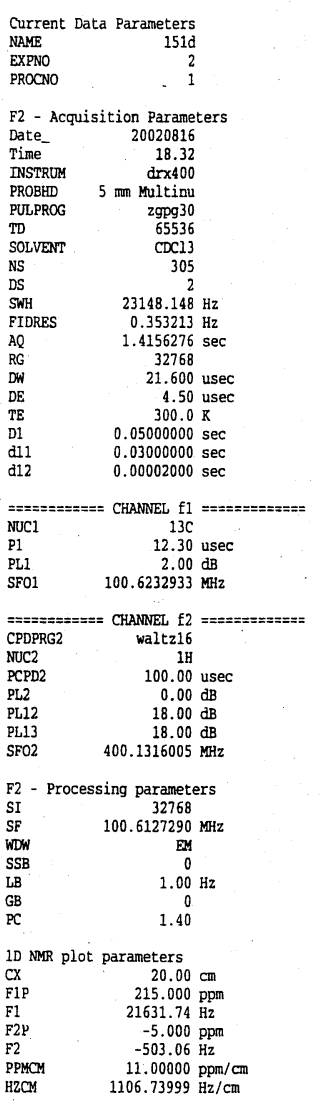

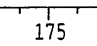

150

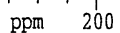

175

125

125

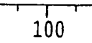

75

${ }_{50}^{1}$

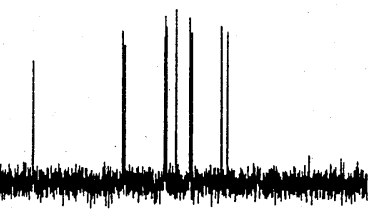




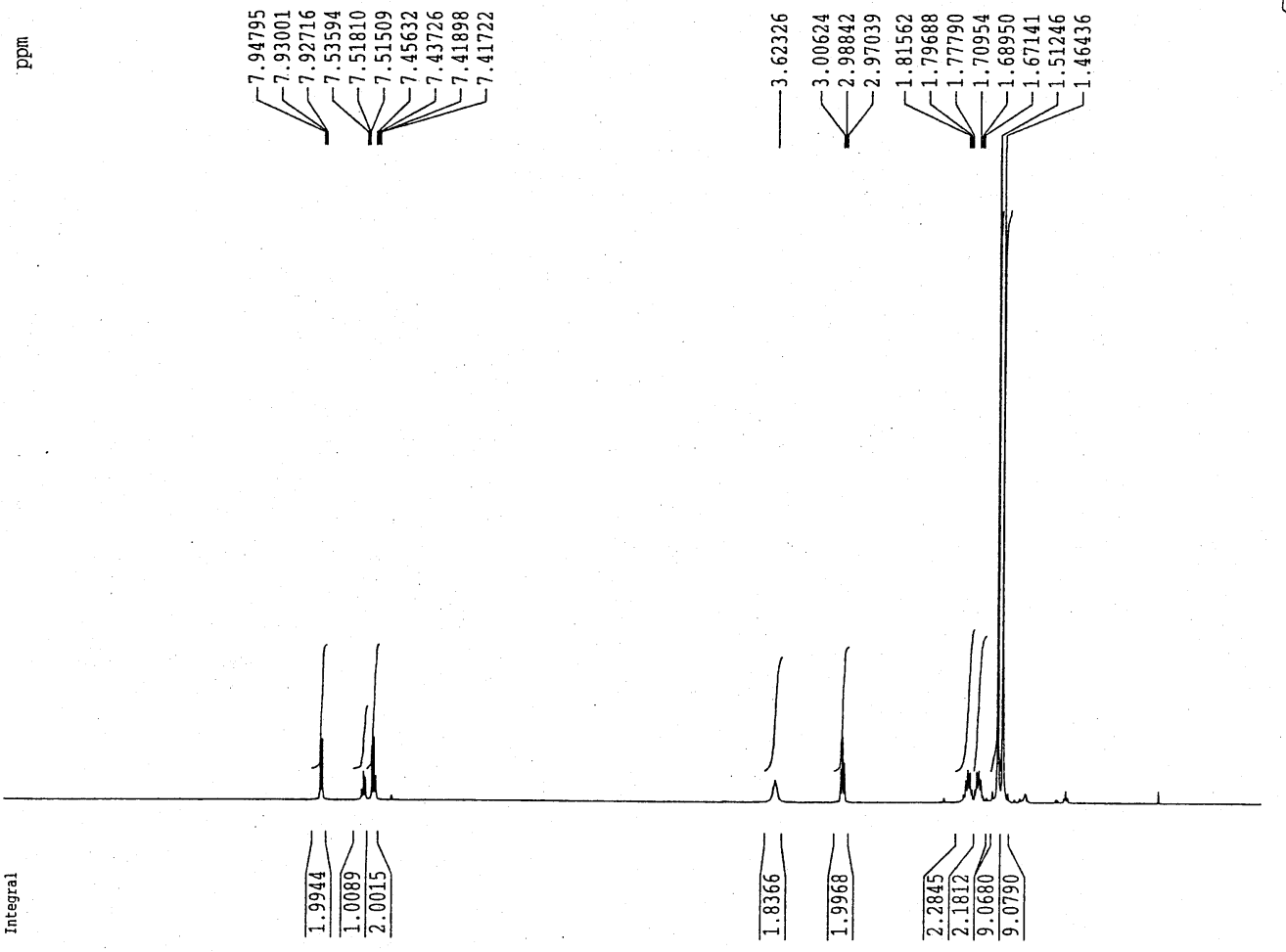

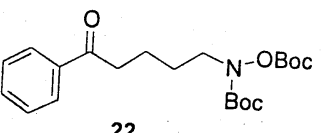

22

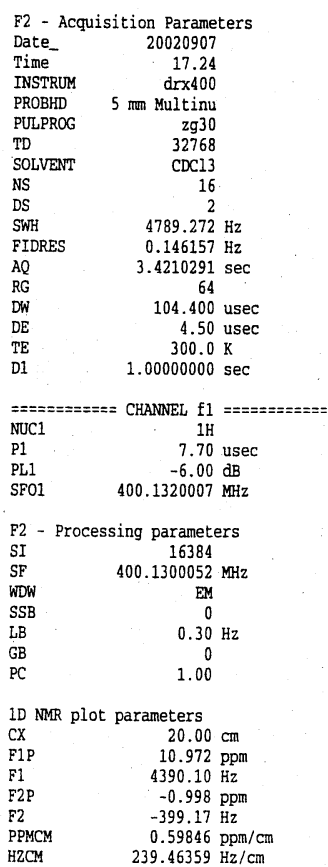

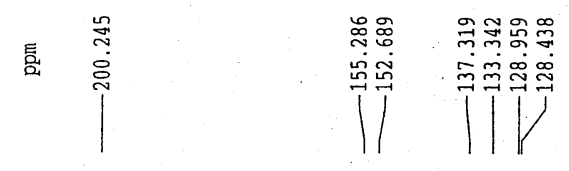

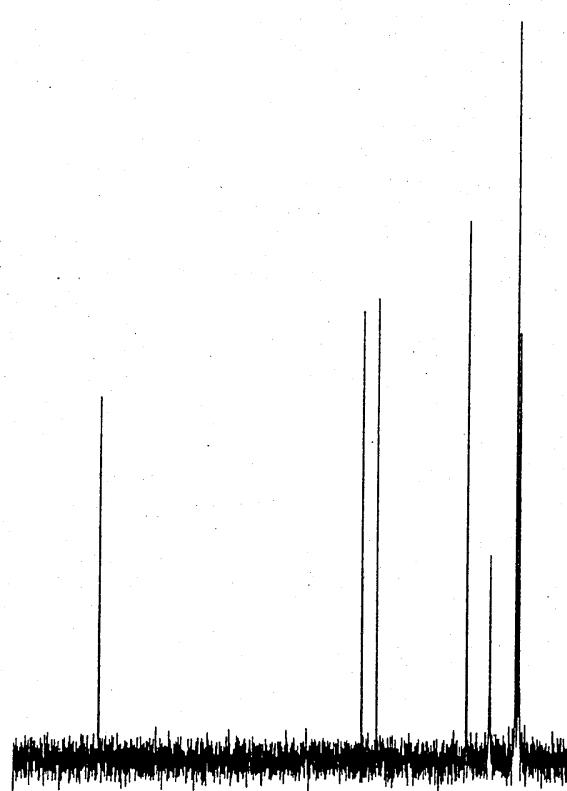

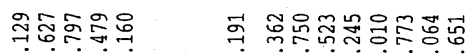

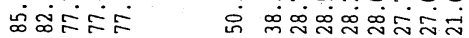

11

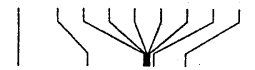

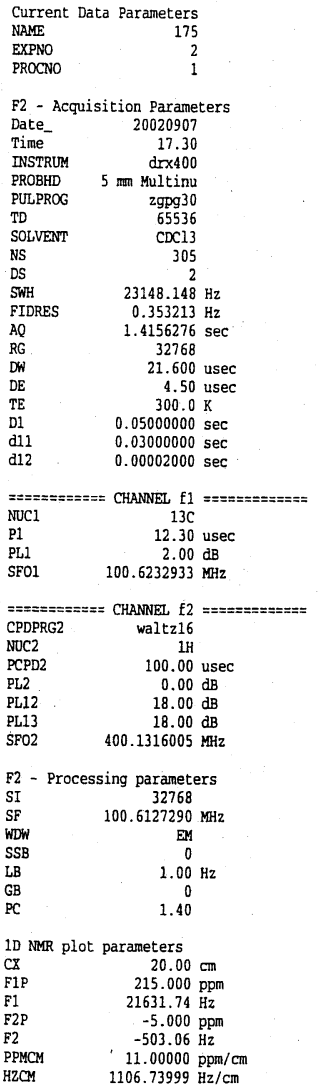

ppm 200

175

150

${ }_{125}^{1}$

100

75

50

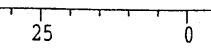



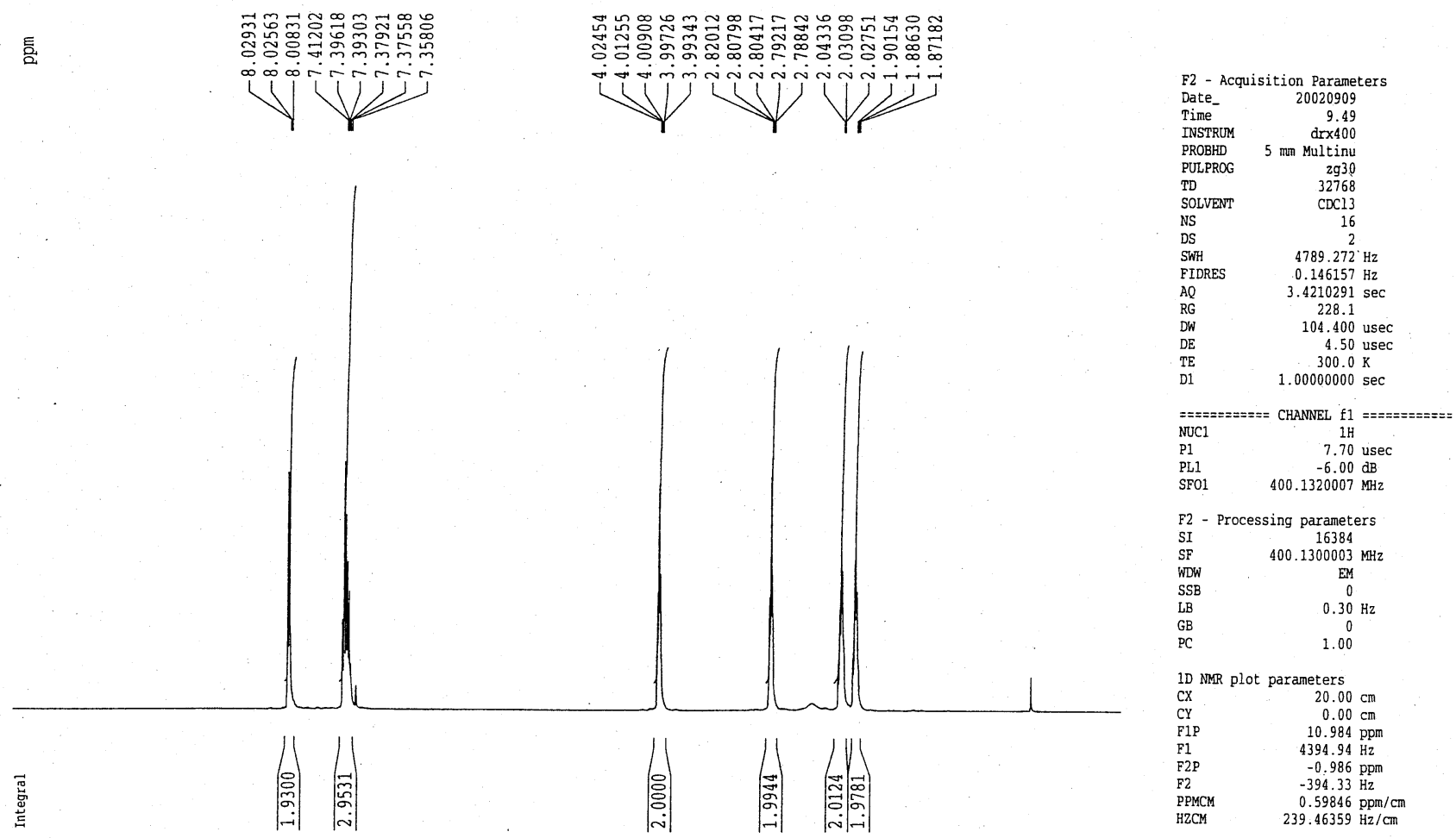

pp

ppm 200

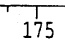

150

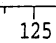

100
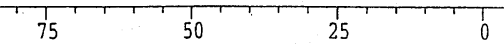


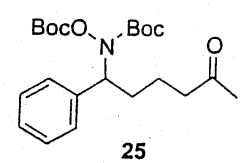

\section{镸}
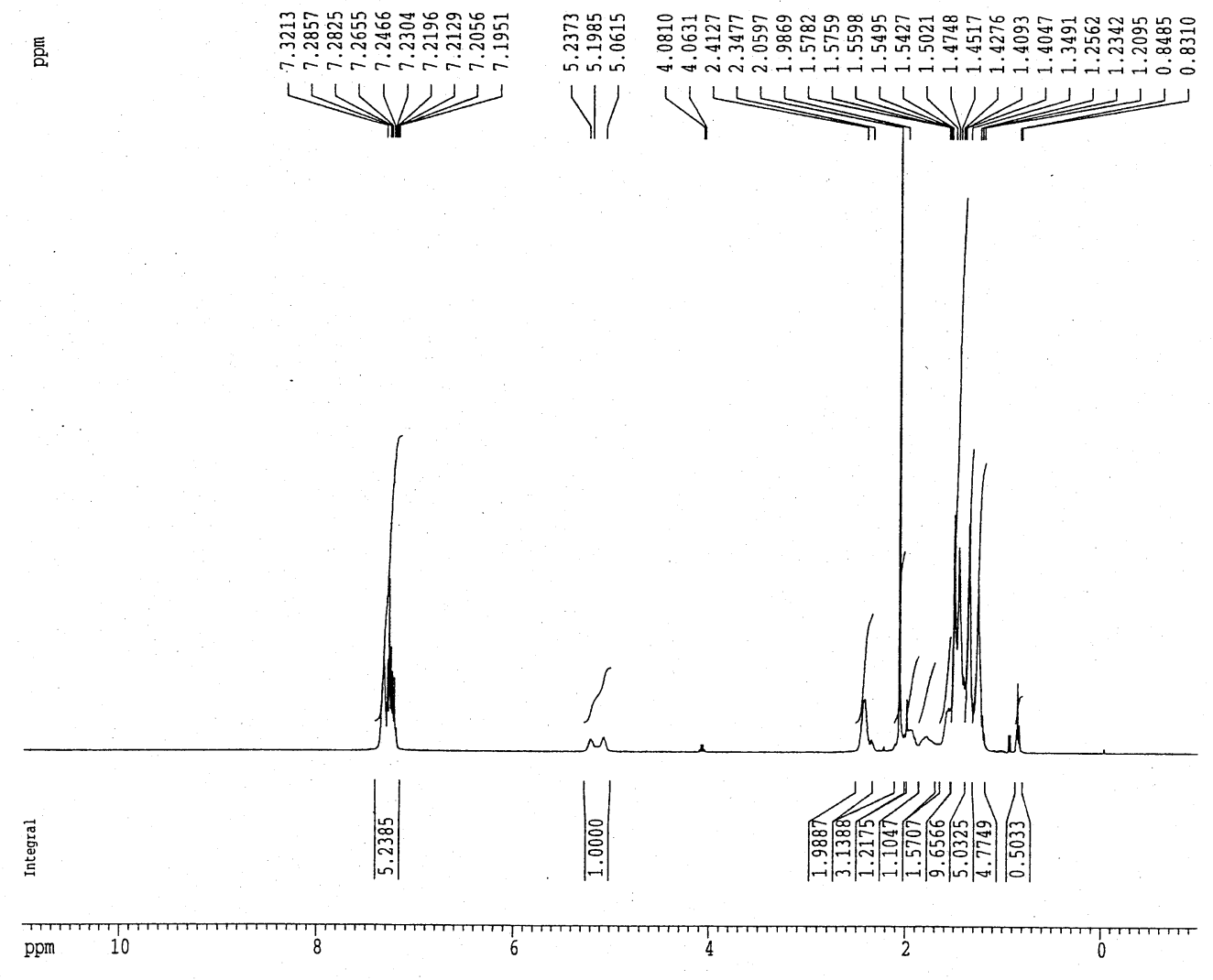

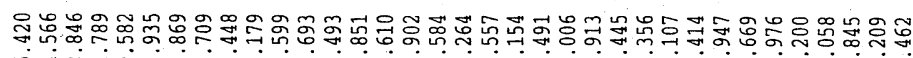

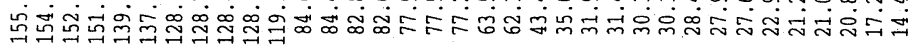

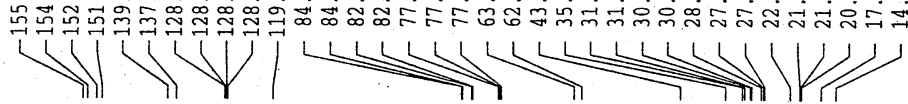

तो

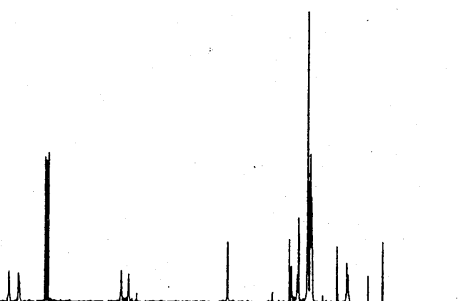

ppm 225

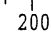

175

150

150

125

100

$\frac{1}{75}$

50

25

- n

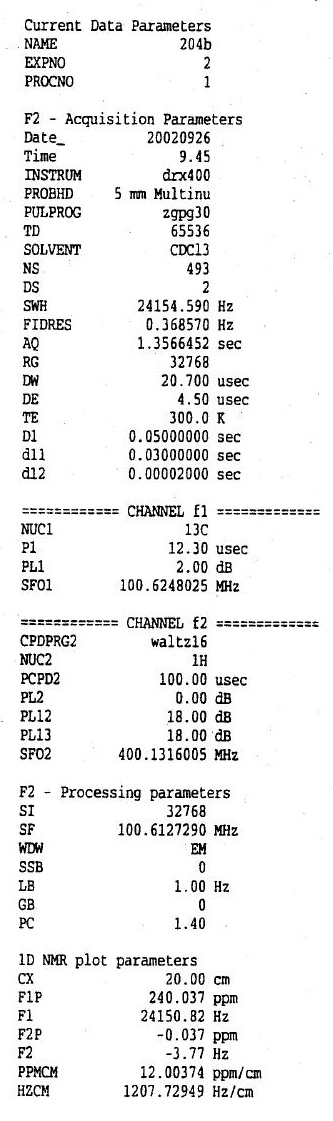



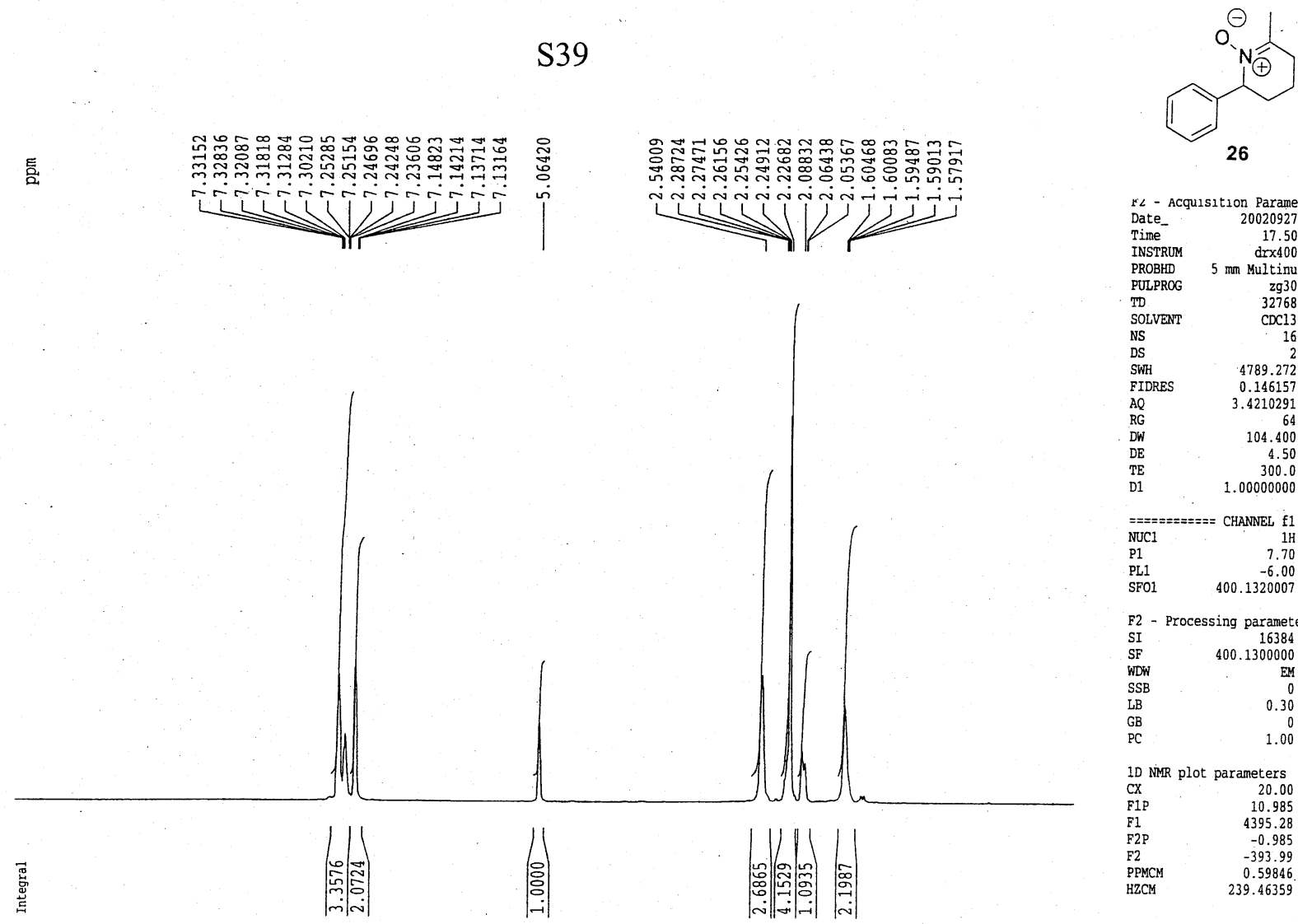

26
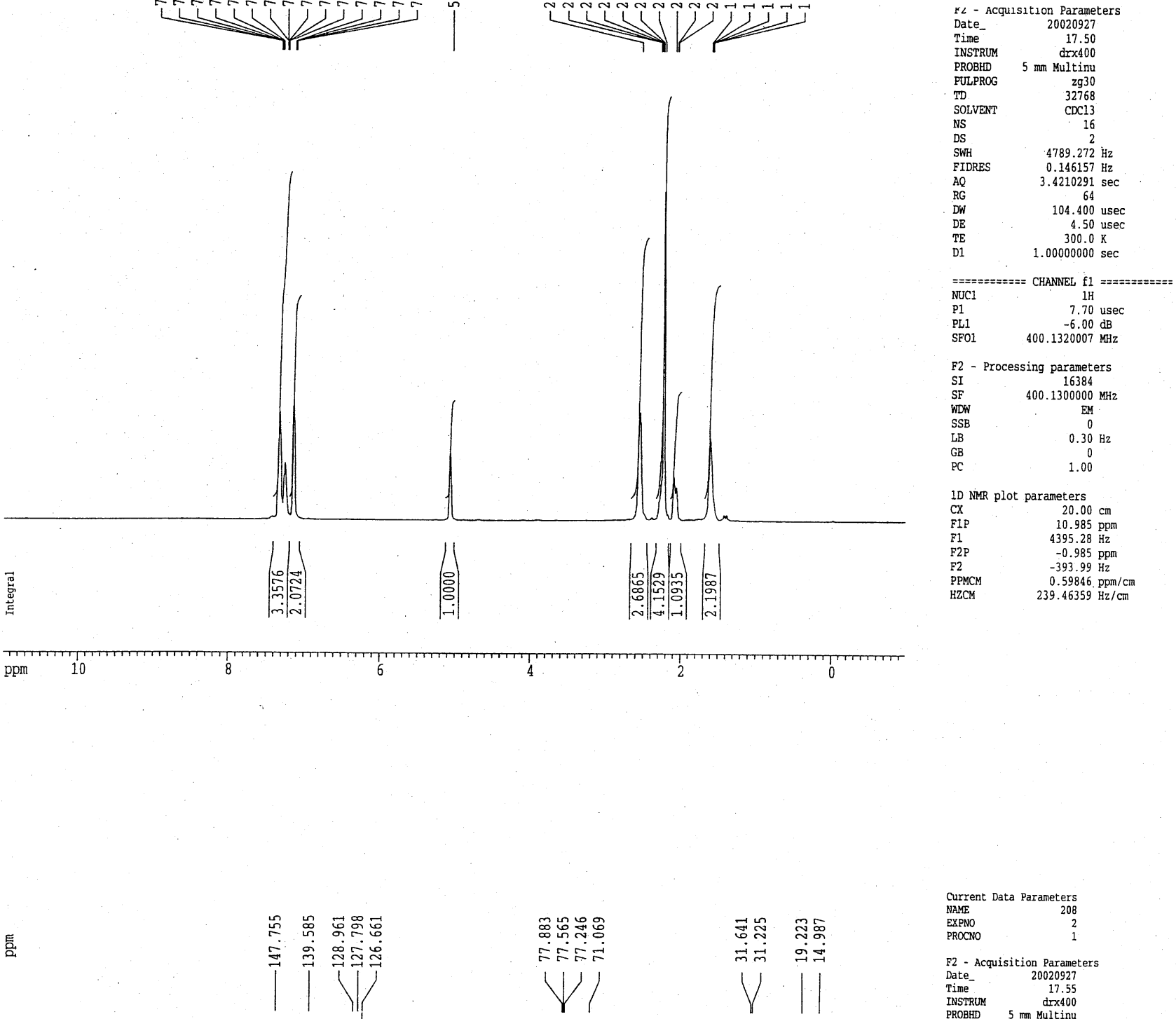

Current Data Parameters
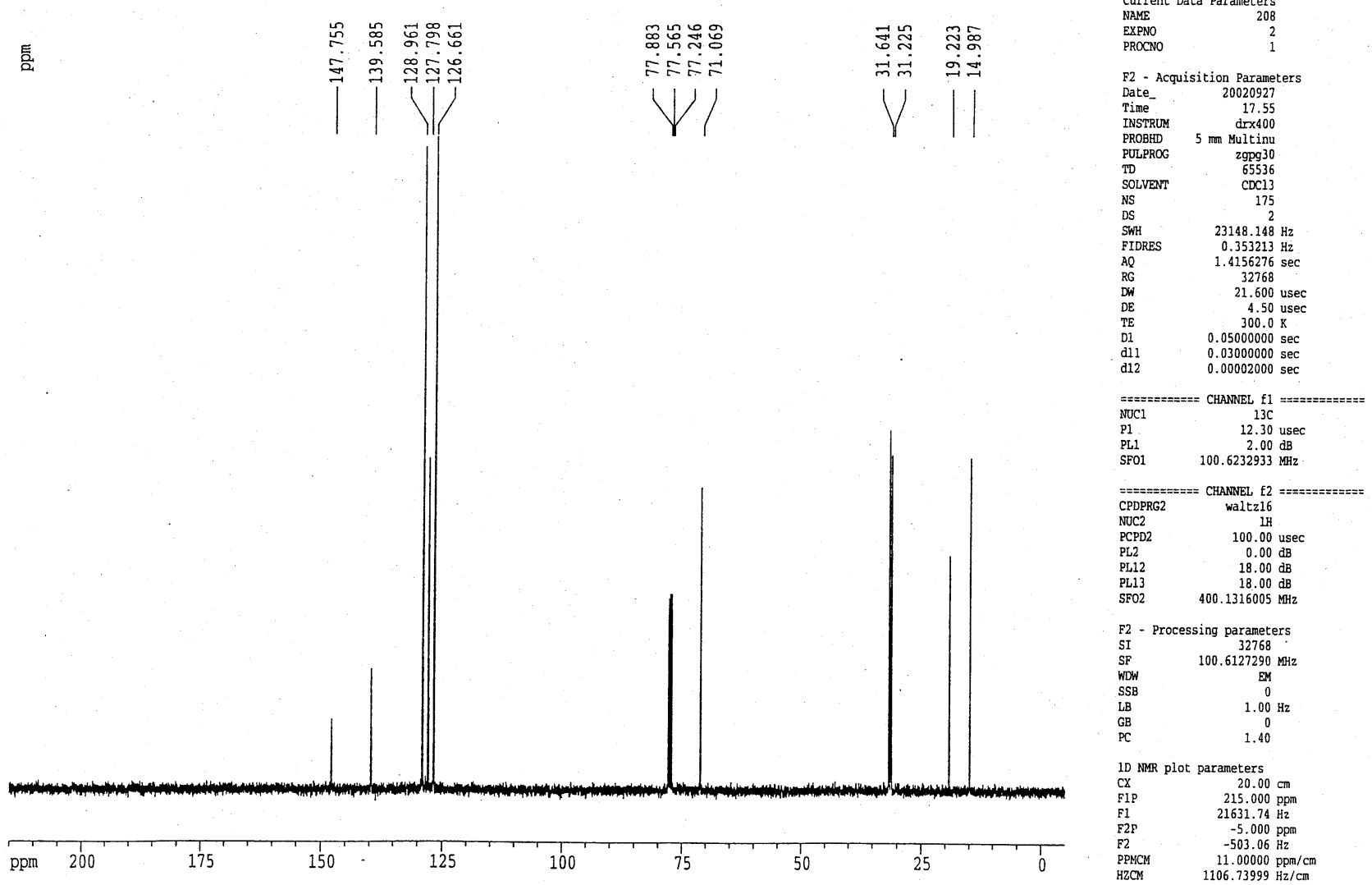


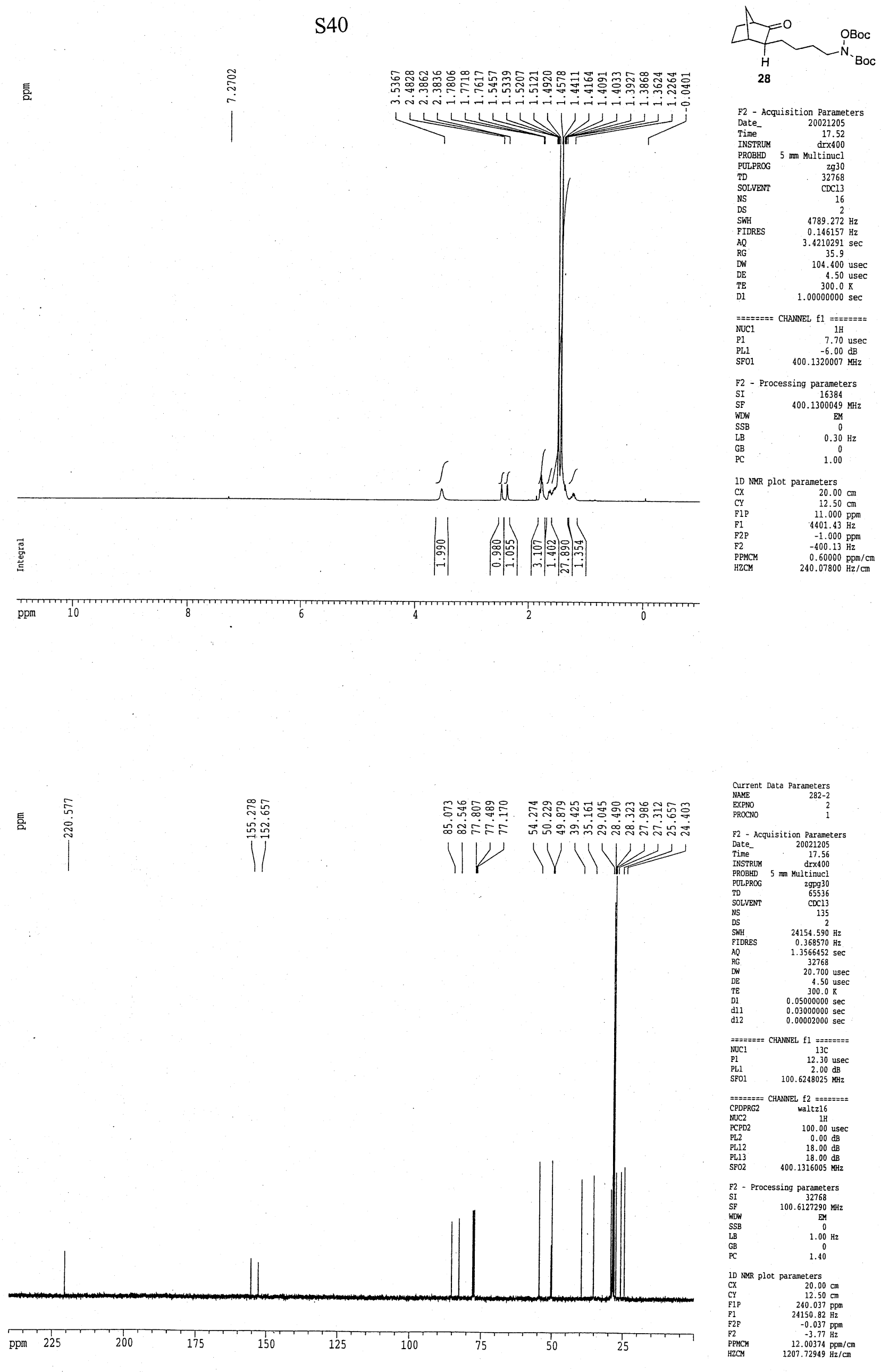




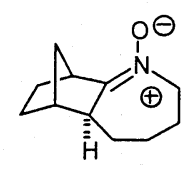

29
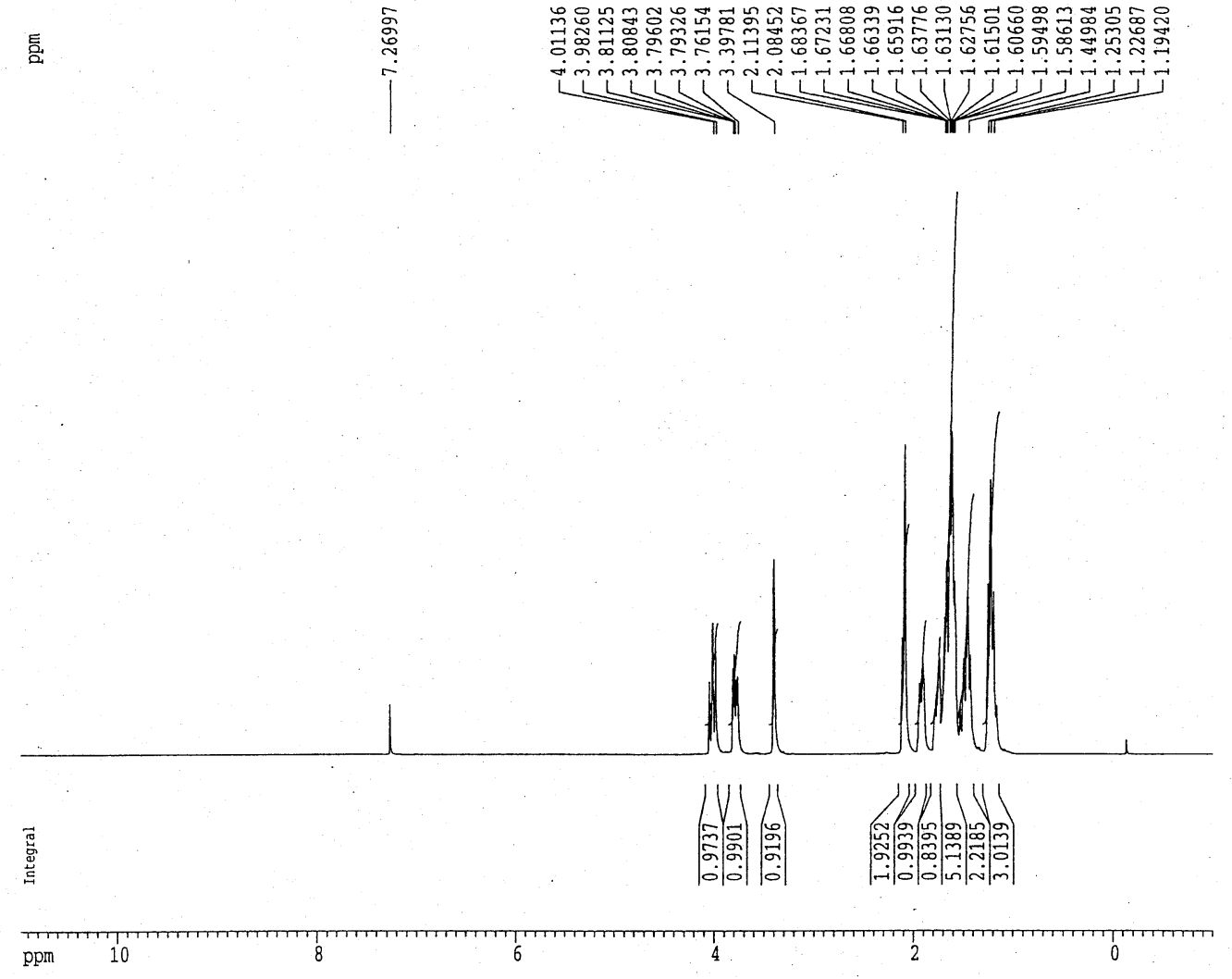

Date_ $\quad 20021204$

Time

PROBHD

PULPROG

TD

NS

DS
SWH
FIDRES

FIDRES

$\mathrm{AQ}$
$\mathrm{RG}$

RG
DW
DE
TE

32768
CDC13

$\begin{array}{lr}\text { WDW } & 400.130069 \\ \text { SSB } & \text { EM } \\ \text { LB } & 0\end{array}$

$\mathrm{PC}$

$\mathrm{HZCM}$

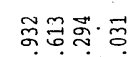

도용

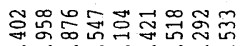

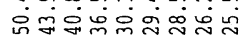

11

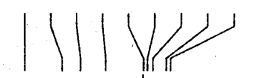

F2 - Acquisition Parameters

drxi400
min Multinucl

16
2.
$4789.272 \mathrm{~Hz}$

$4789.272 \mathrm{~Hz}$
$0.146157 \mathrm{~Hz}$

$3.4210291 \mathrm{se}$

40.3
104.400 usec

4.50 usec

$300.0 \mathrm{~K}$
$1.00000000 \mathrm{sec}$

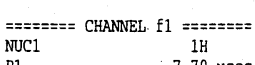

$\begin{array}{ll}\text { NUC1 } & 1 \mathrm{H} \\ \mathrm{P1} & 7.70 \mathrm{usec}\end{array}$

SF01 400.1320007

F2 - Processing parameters

$\begin{array}{lc}\text { SI } & 16384 \\ \mathrm{SF} & 400.1300069 \mathrm{MHz}\end{array}$

$\begin{array}{lc}\text { SSB } & 0 \\ \text { LB } & 0.30 \mathrm{~Hz} \\ \text { GB } & 0\end{array}$

1.00

1D NMR plot parameters
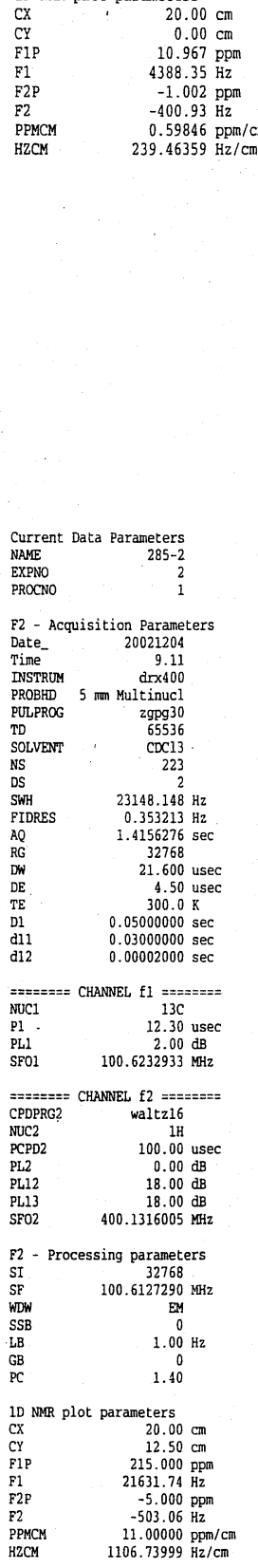

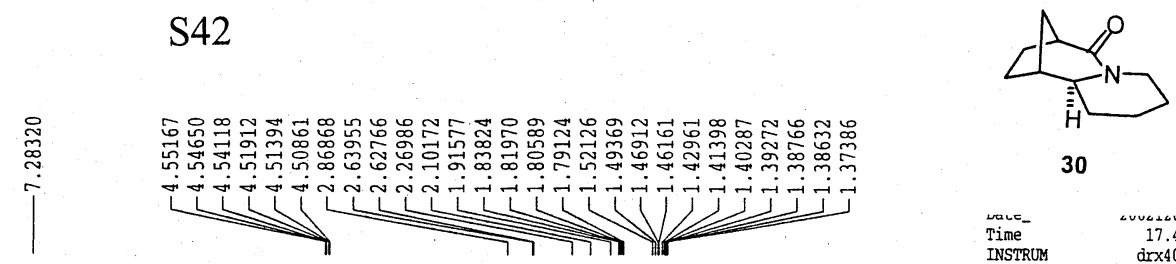

30

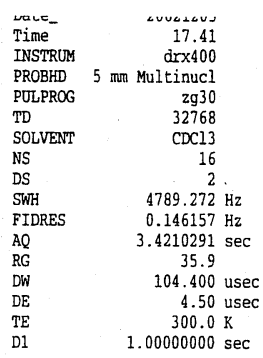

$===== \pm=$ CHANNEL f1 $=======$

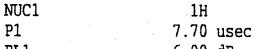

$\begin{array}{lr}\text { P1 } & -6.00 \mathrm{~dB} \\ \text { PL1 } & 400.1320007\end{array}$

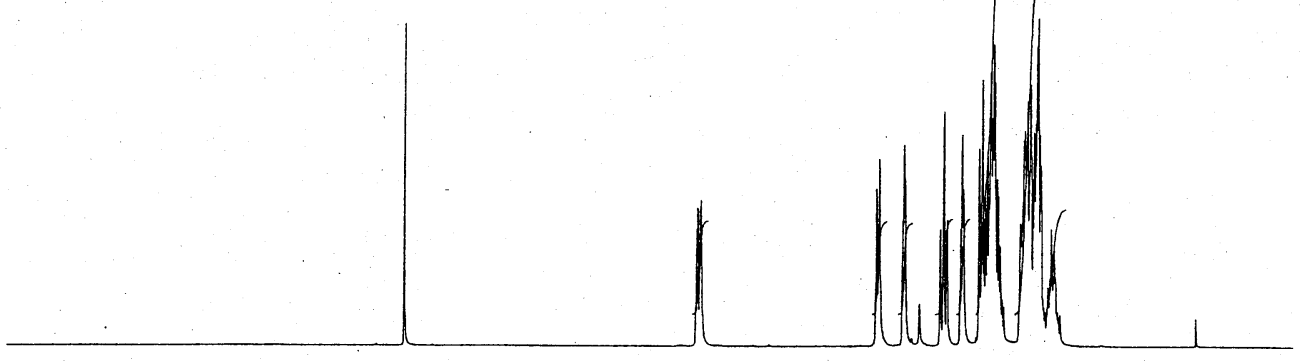

F2 - Processing parameters

$\begin{array}{lc}\text { F2 } & \text { Processing parameters } \\ \text { SI } & 16384 \\ \text { SF } & 400.1300000 \mathrm{MHz}\end{array}$

WDW $\quad$ EM

$\begin{array}{lc}\text { LB } & 0.30 \mathrm{~Hz} \\ \text { GB } & 0\end{array}$

1.00

1D NMR plot parameters

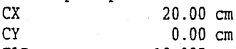

$\begin{array}{ll}\text { CY } & 10.00 \mathrm{~cm} \\ \text { F1P } & 10.985 \mathrm{ppm}\end{array}$

F1 $\quad 4395.28 \mathrm{~Hz}$

F2 $\quad-0.985 \mathrm{ppm}$

$\begin{array}{lr}\text { PPMCM } & 0.59846 \mathrm{ppm} / \mathrm{cm} \\ \text { HZCM } & 239.46359 \mathrm{~Hz} / \mathrm{Cm}\end{array}$
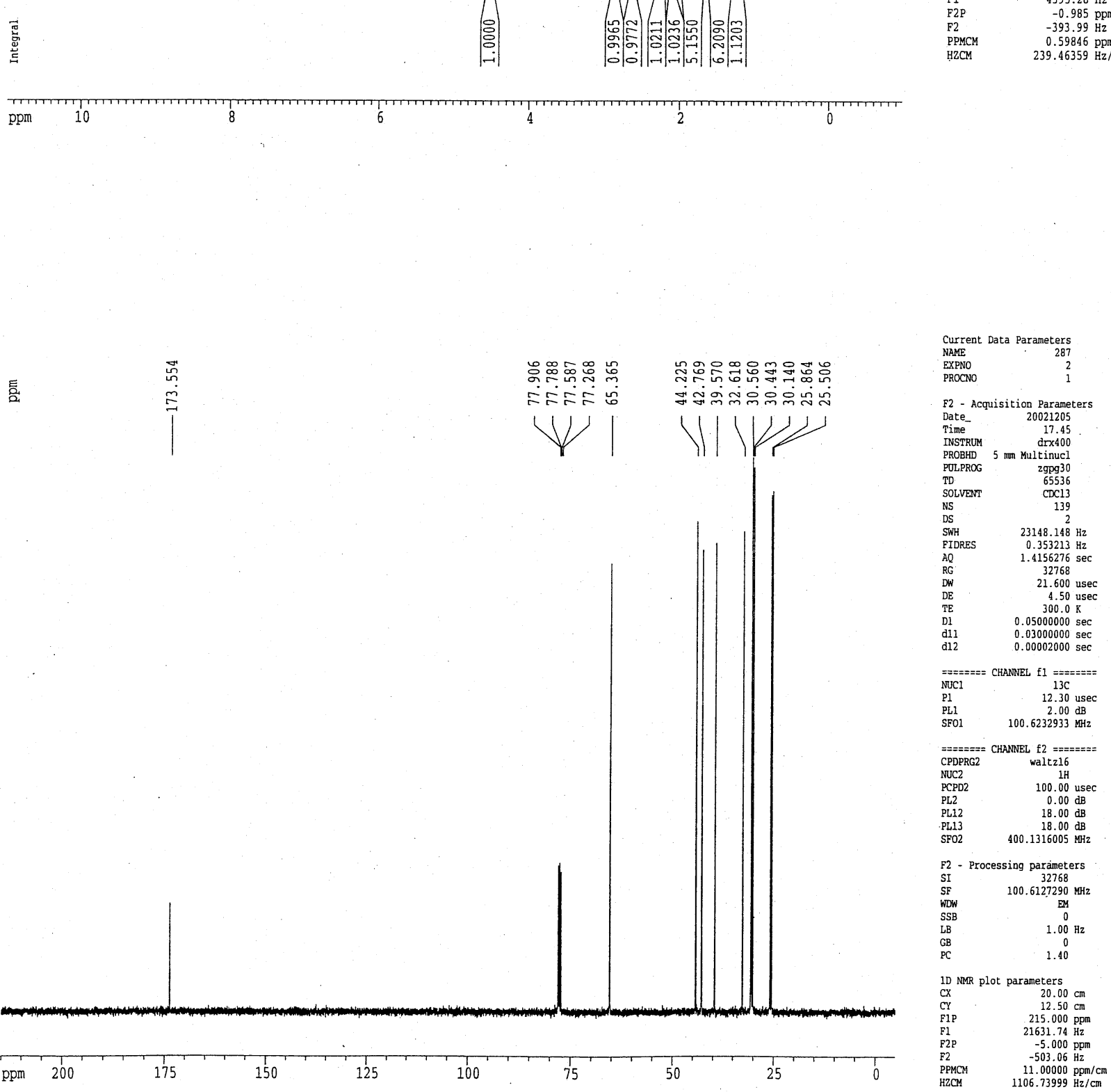

镸

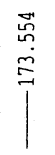

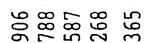

도은

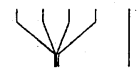

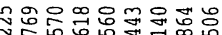

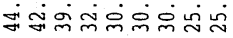

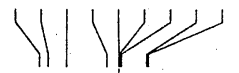

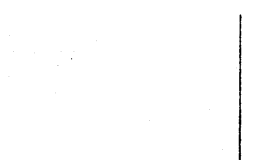

175

150

125

100

75

50

25 
長
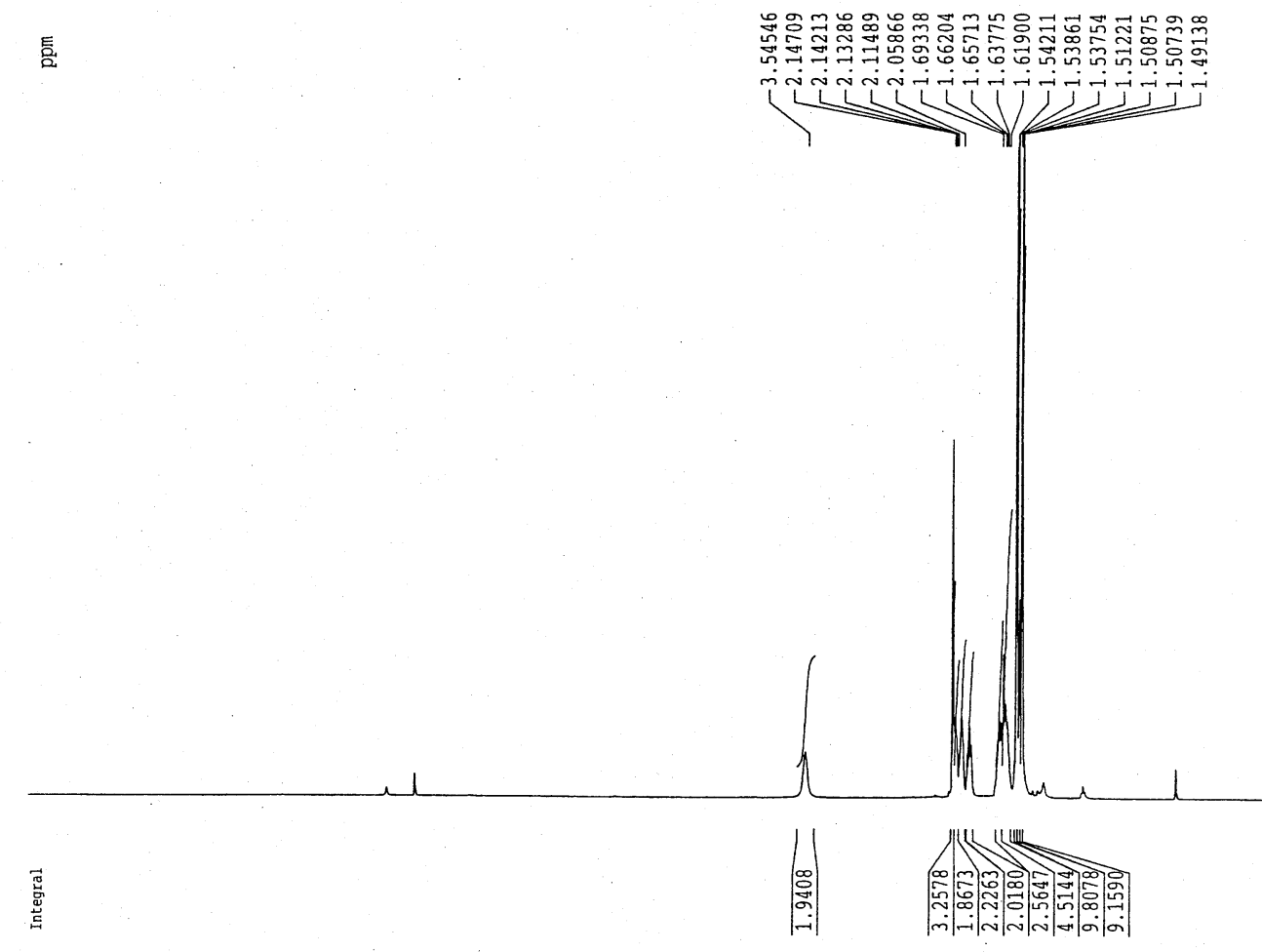

$\mathrm{ppm}$

ppm 10

镸

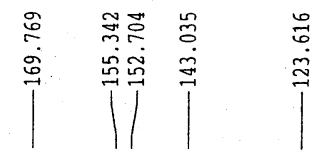

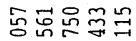

ก่ำำ

IIK

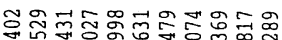

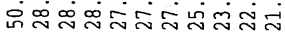

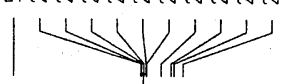

(1)

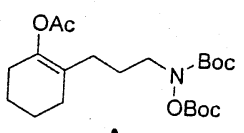

A

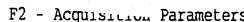

2 - Acquisslivu Parameters

Time $\quad 20030608$

INSTRUM drx400

PROBPROG 5 mm Multinucl

SOLVENT

NS

SWH $\quad 4789.272 \mathrm{~Hz}$

FDRES $\quad 0.146157 \mathrm{~Hz}$

$\begin{array}{lc}\text { AQ } & 3.4210291 \mathrm{sec} \\ \text { RG } & 101.6\end{array}$

104.400 usec

4.50 usec

$\begin{array}{ll}\mathrm{TE} & 300.0 \mathrm{~K} \\ \mathrm{D} 1 & 1.00000000 \mathrm{sec}\end{array}$

$=======$ CHANNEL $f 1$ = $=====$

$\begin{array}{ll}\text { NUC1 } & 1 \mathrm{H} \\ \text { P1 } & 7.70 \text { usec }\end{array}$

$\begin{array}{lr}\text { PL1 } & -6.00 \mathrm{~dB} \\ \text { SF01 } & 400.1320007 \mathrm{MHz}\end{array}$

F2 - Processing parameters

SI 16384

$\begin{array}{cc}\text { SF } & 400.1300000 \mathrm{MHz} \\ \text { WDW } & \text { EM } \\ \text { SSB } & 0\end{array}$

$\begin{array}{lc}\text { SSB } & 0 \\ \text { LB } & 0.30 \mathrm{~Hz} \\ \text { GB } & 0\end{array}$

1D NMR plot parameters

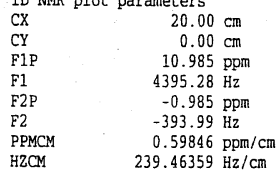


S44

OBoc

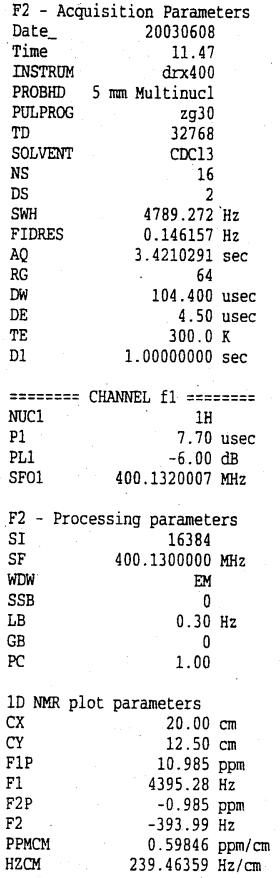

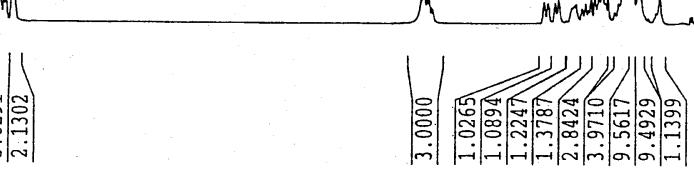
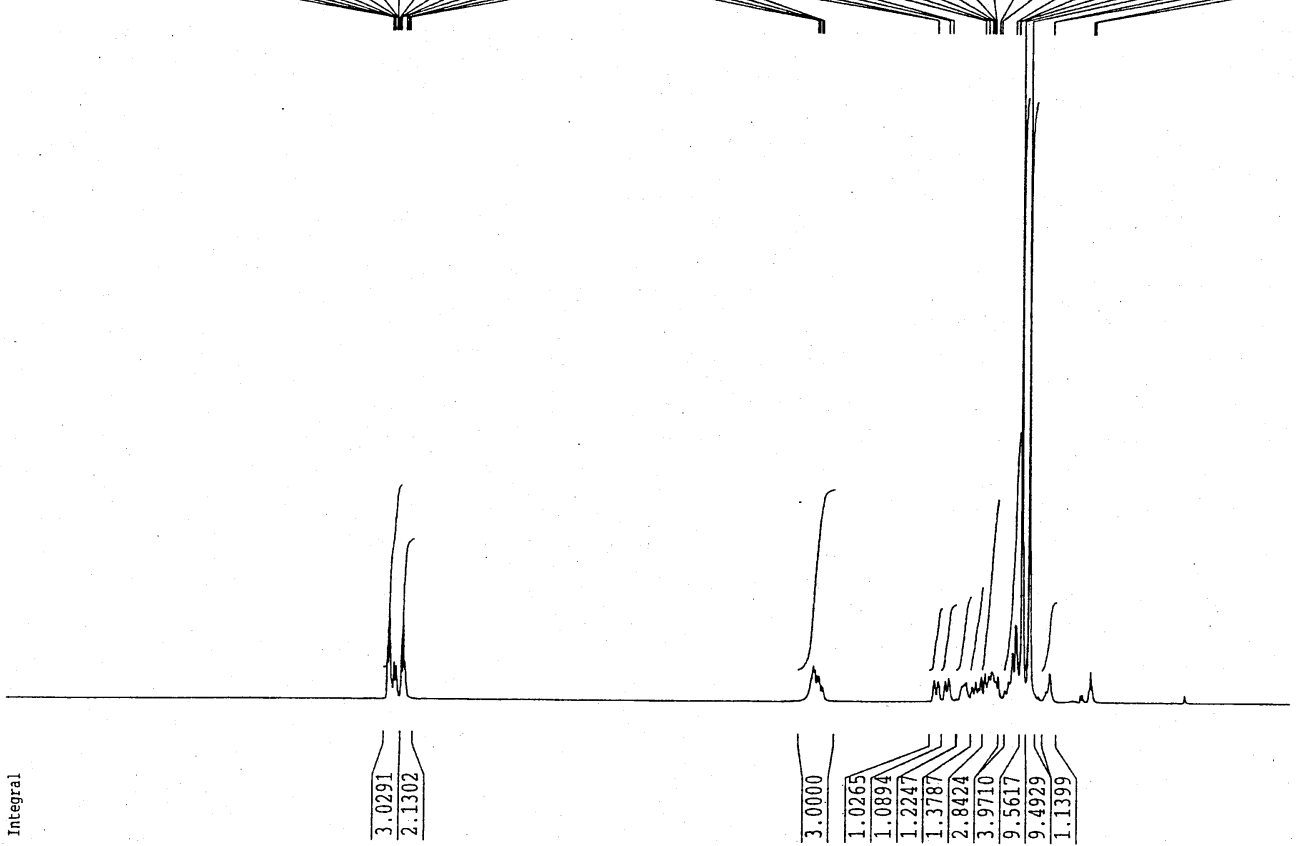

ppm 10

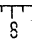

듬
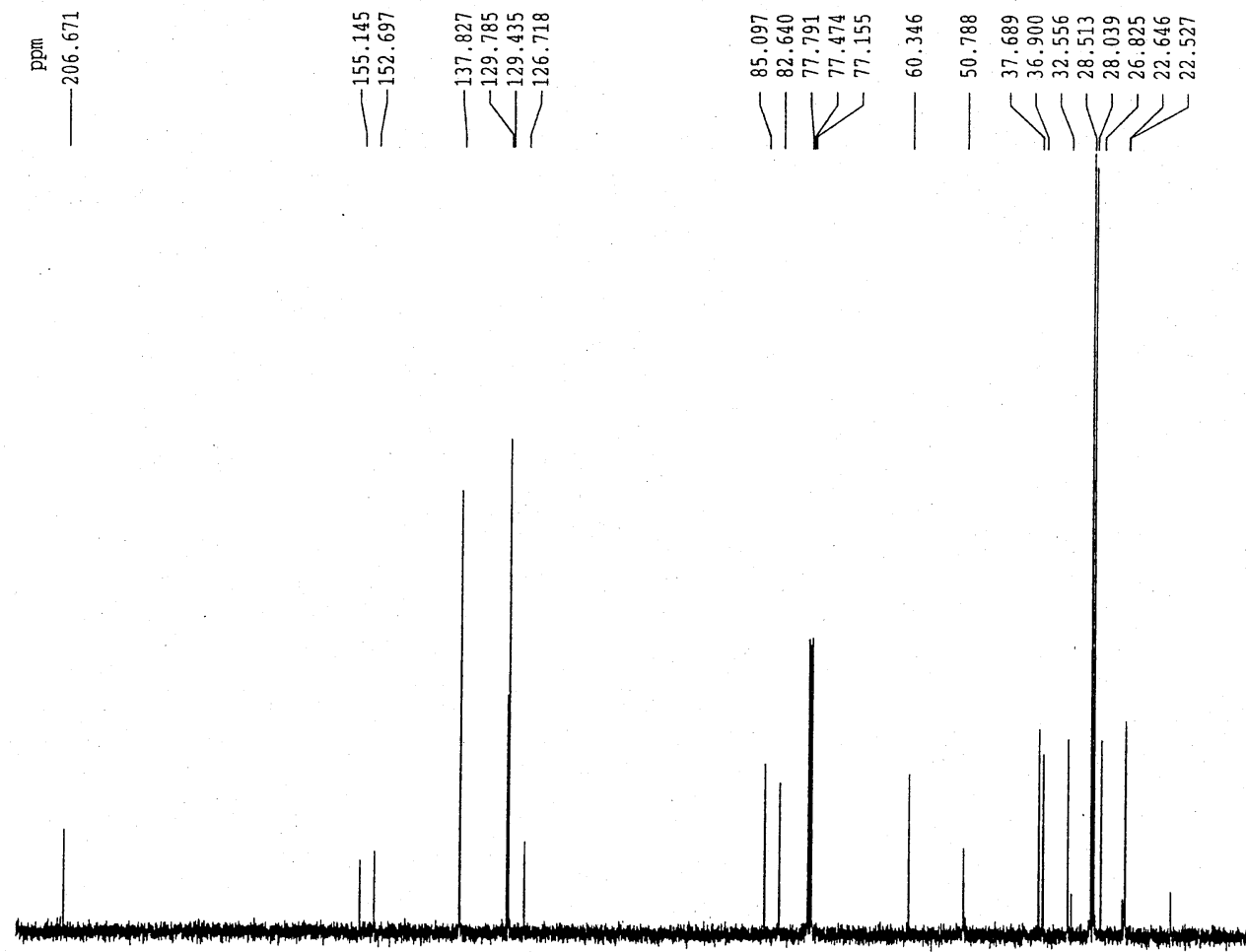

ppm

ppm 200

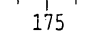

150

125
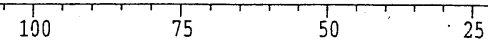

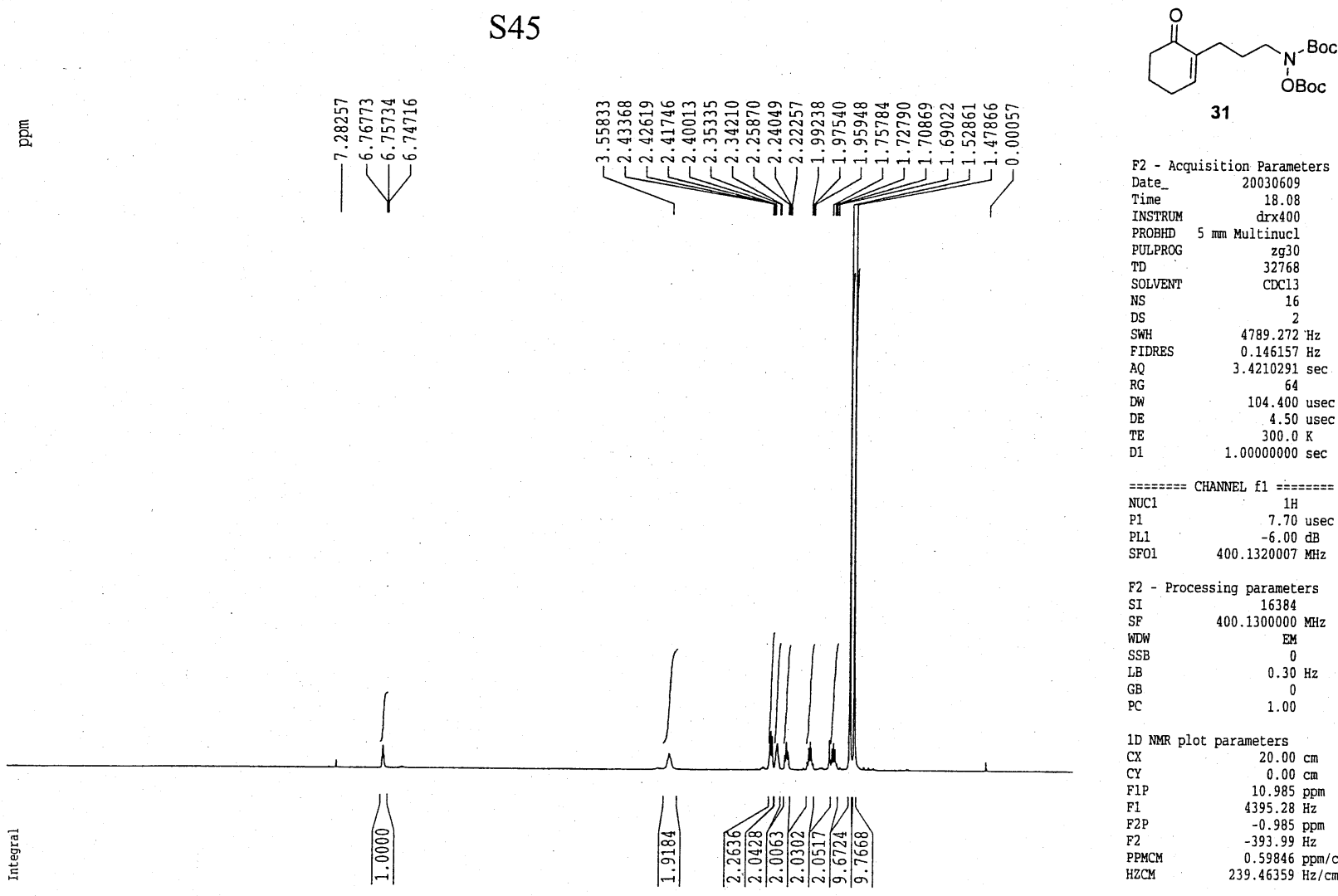

31
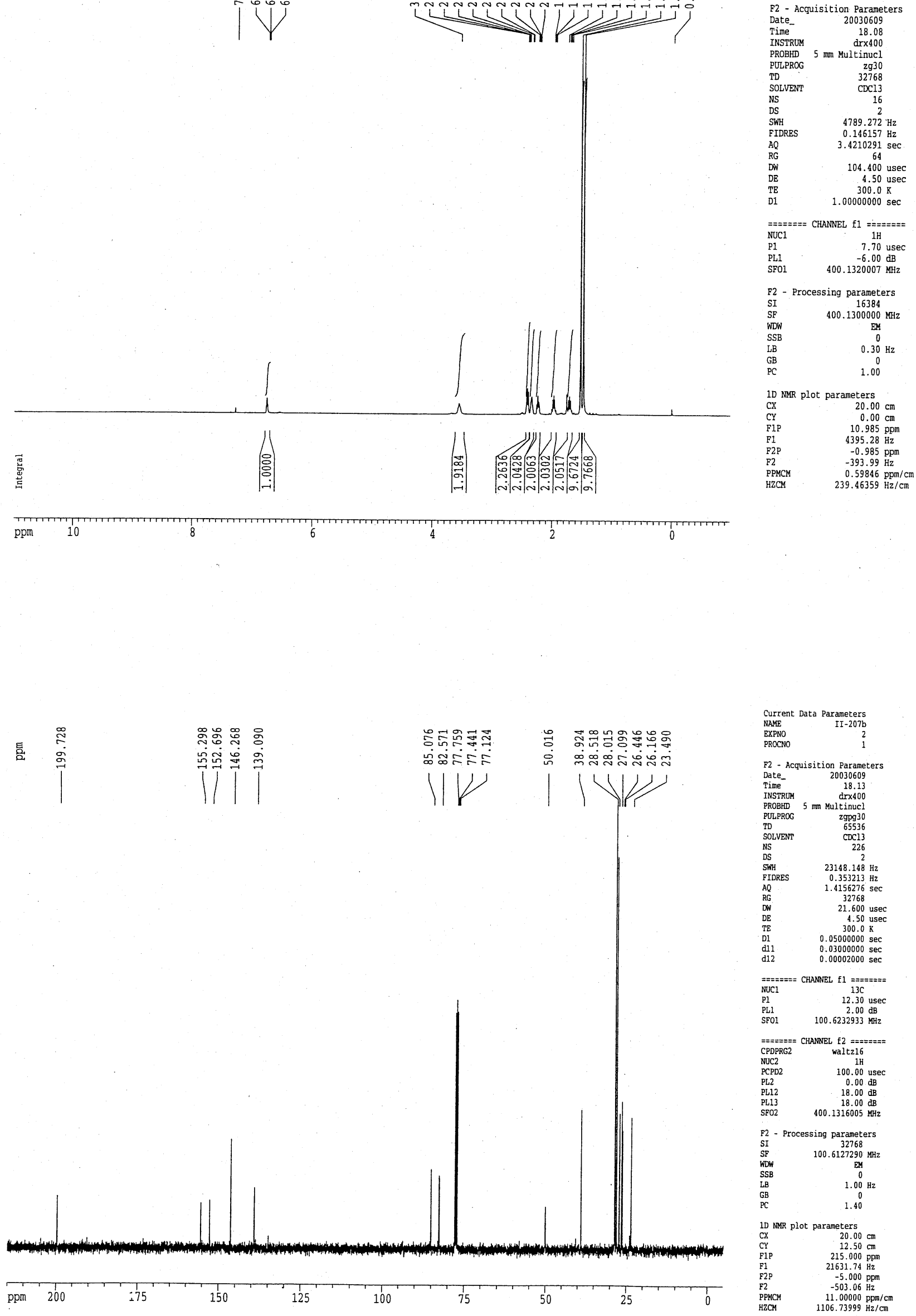

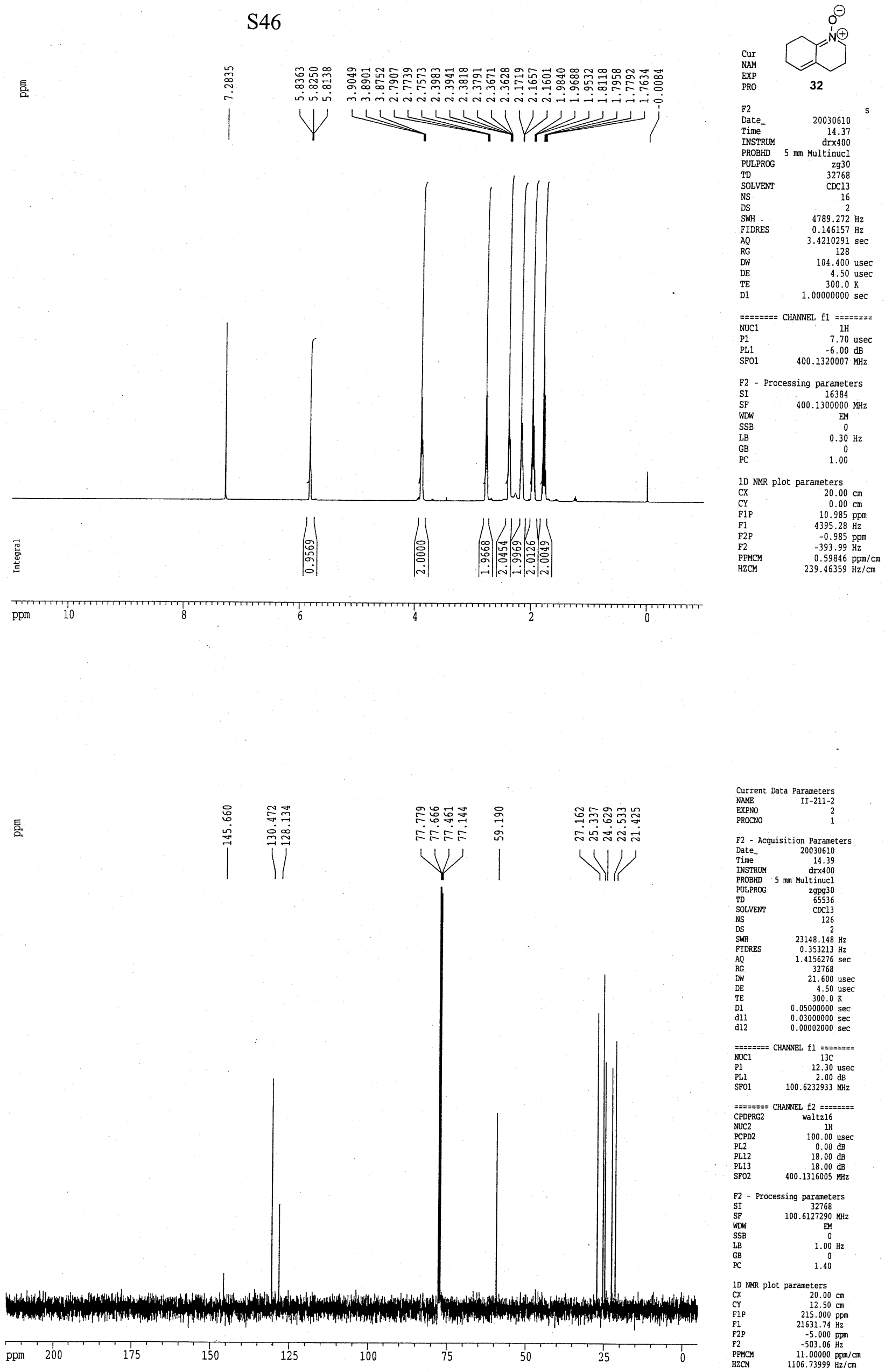


\section{S47}
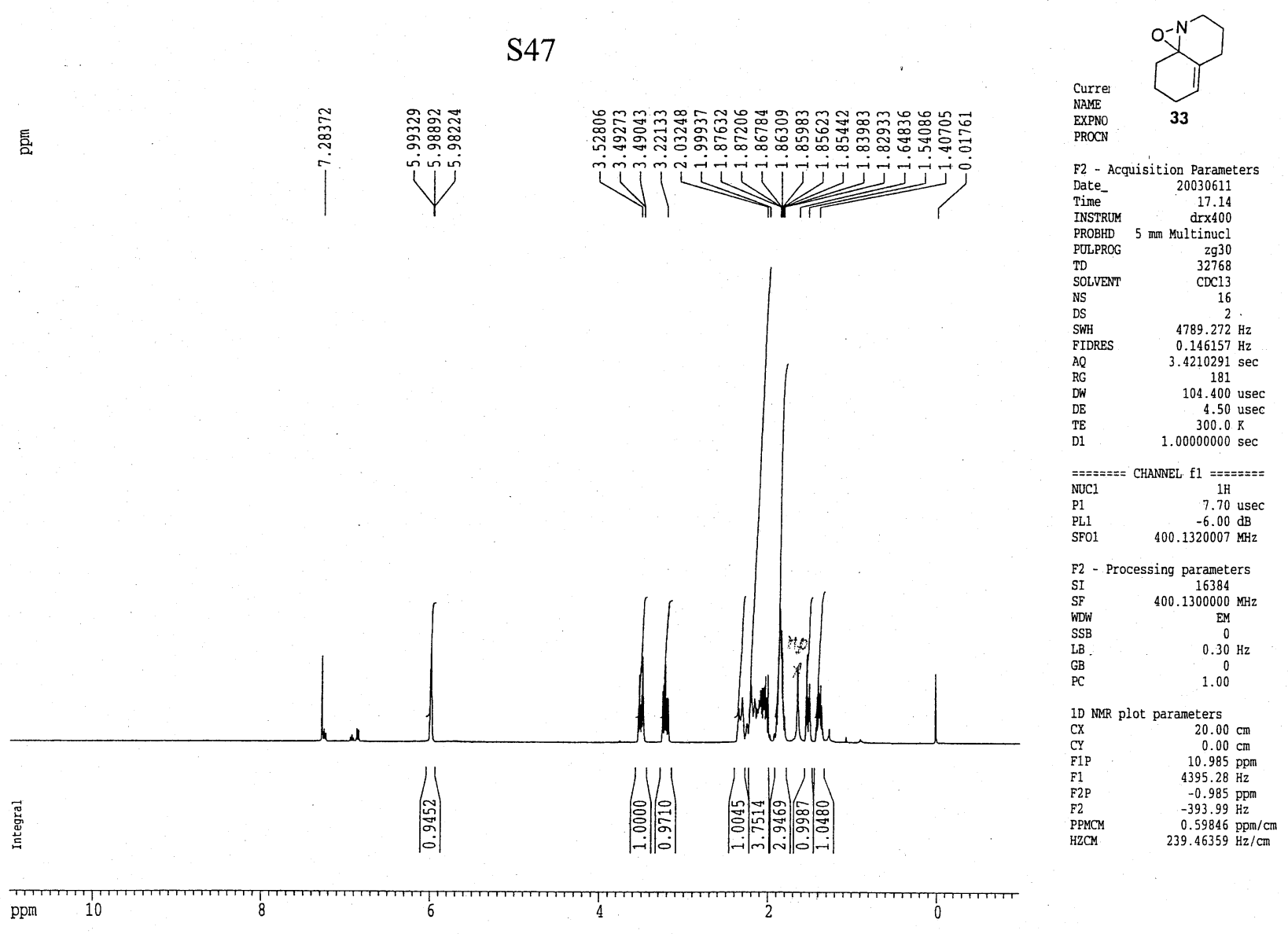

镸

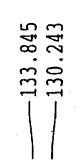

긍윢명

政兵

11

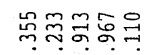

mi்

111

ppm 225

200

175

175
150

: 12

125

5

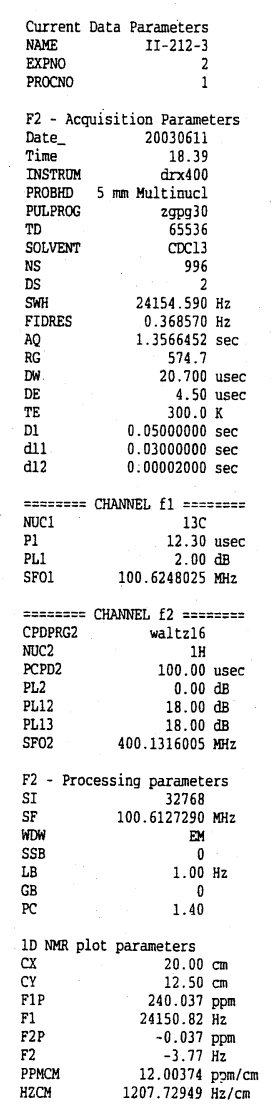



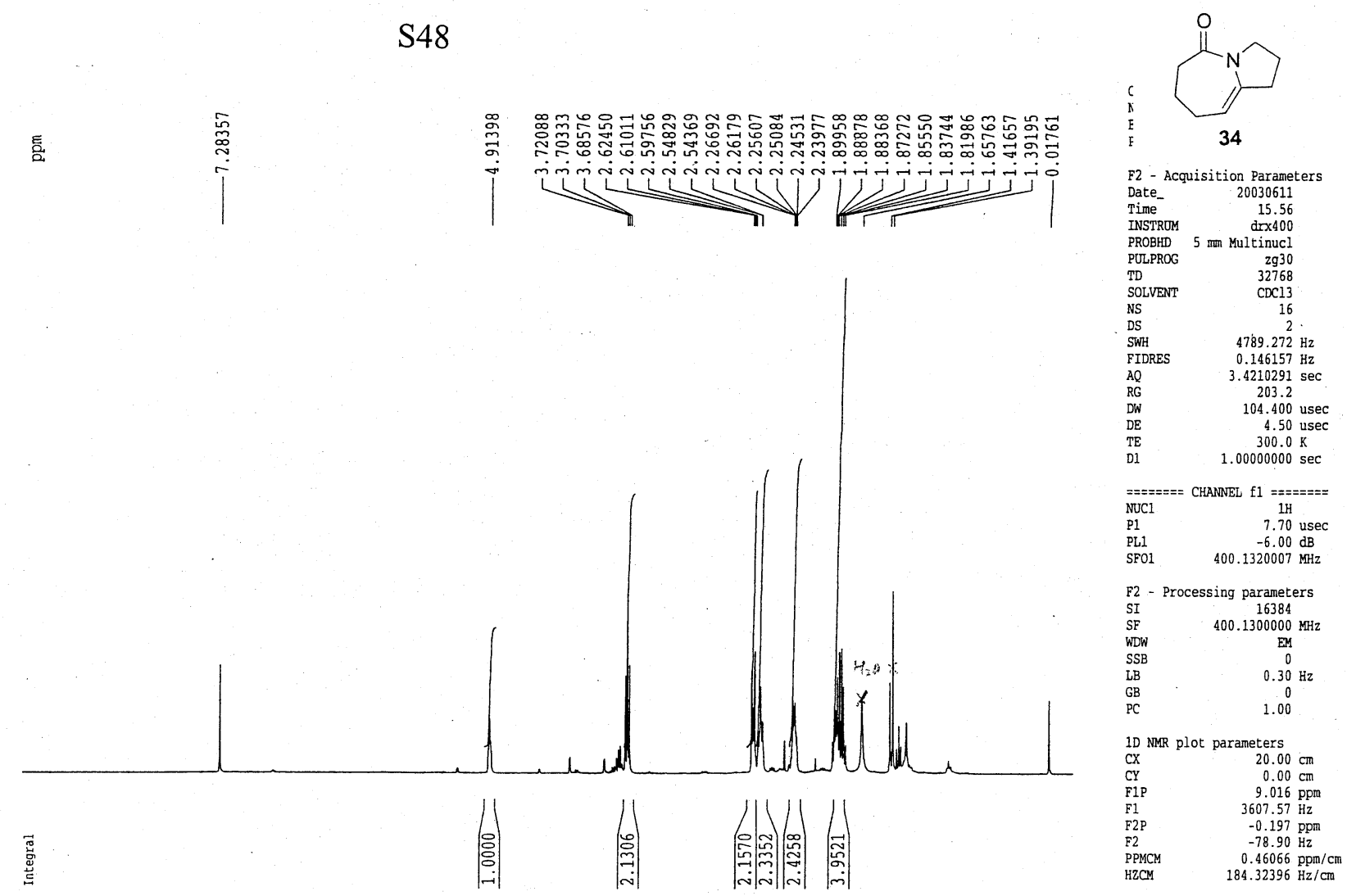

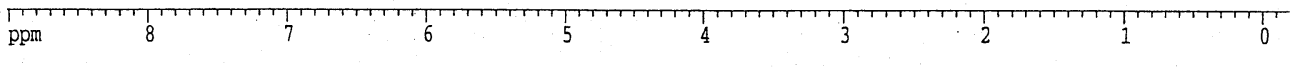

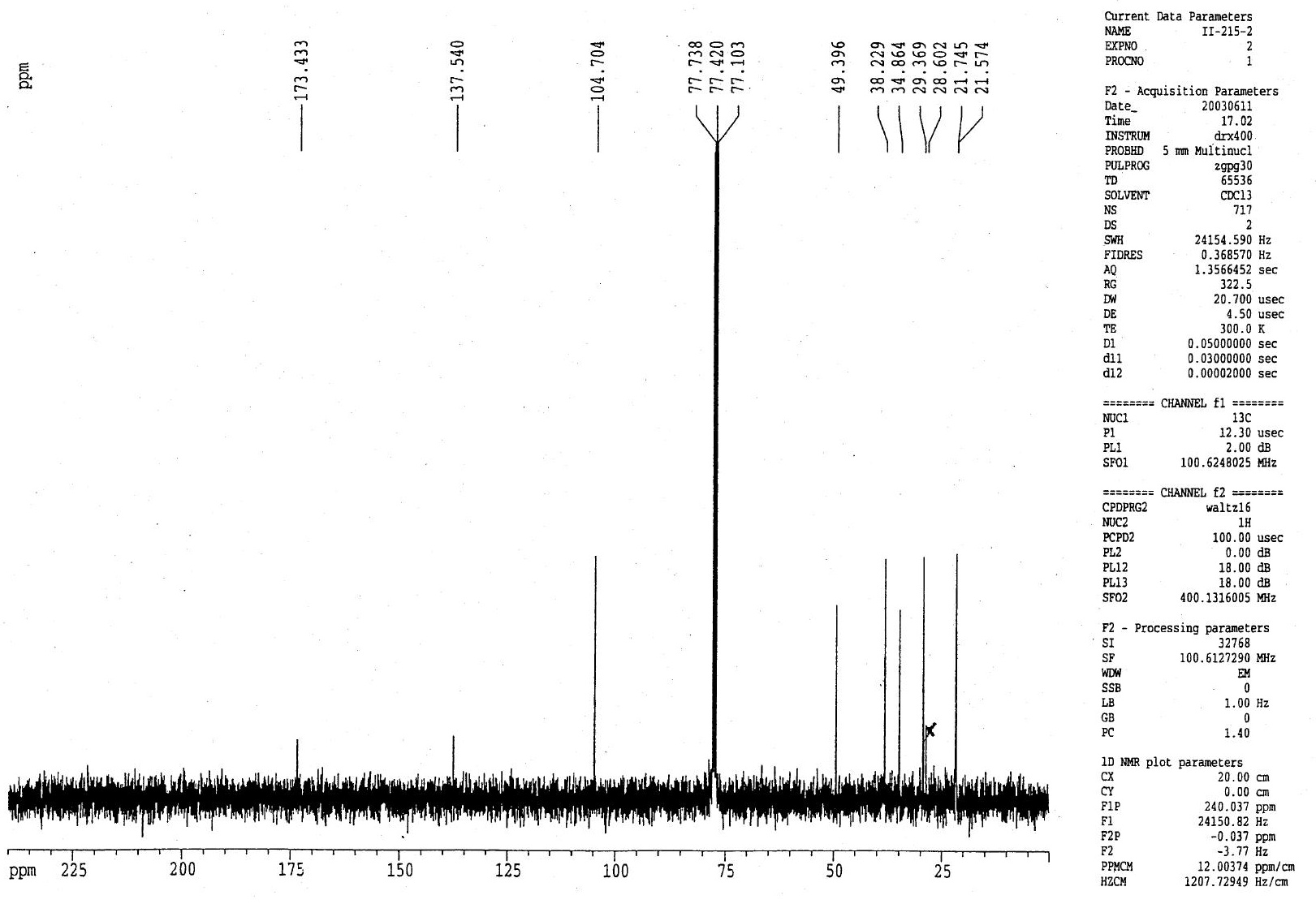

